•生物编目・2020年新物种专题

\title{
中国膜翅目2020年新分类单元
}

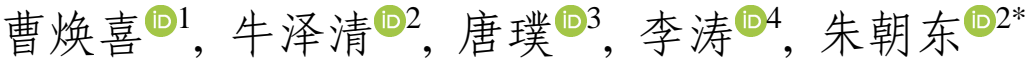

1. 中国科学院动物研究所国家动物标本资源库, 北京 100101; 2. 中国科学院动物研究所动物进化与系统学重点实验室, 北京 100101;

3. 浙江大学农业与生物技术学院, 杭州 310058; 4. 国家林业和草原局森林和草原病虫害防治总站, 沈阳 110034

摘要: 本文对中国膜翅目昆虫2020年发表的新分类单元进行了梳理和总结。结合数据库检索和统计表格征集，基于采自中国 的膜翅目昆虫标本记录, 全世界膜翅目学者于 2020 年共发表涉及中国膜翅目分类单元的期刊论文104篇、出版1本图书专著, 分类单元新增及变动条目共 469 条: 4 个新属(其中 1 个绝灭属), 1 个新亚属, 321 个新种(其中 10 个绝灭种), 8 个新组合, 22 个新异 名, 1 个中国新记录亚科, 18 个中国新记录属, 93 个中国新记录种, 1 个替代名。绝灭类群有 11 条, 涉及3总科 3 科5属; 现生膜翅目 有458条, 涵盖11总科29科170属, 其中6科40属不涉及新种。发表现生膜翅目新种的9总科23科130属: 广腰亚目(Symphyta)叶 蜂总科(Tenthredinoidea) 23 个新种; 细腰亚目(Apocrita)针尾部(Aculeata) 3 个总科(蜜蜂总科(Apoidea) 29 个新种、青蜂总科 (Chrysidoidea) 2 个新种、胡蜂总科(Vespoidea) 20 个新种); 细腰亚目寄生部(Parasitica) 5 个总科(小蜂总科(Chalcidoidea) 23 个新 种、瘷蜂总科(Cynipoidea) 12 个新种、姬蜂总科(Ichneumonoidea) 206个新种、广腹细蜂总科(Platygastroidea) 4 个新种、钩腹 蜂总科(Trigonalyoidea) 2 个新种)。2020年发表的所有中国膜翅目新种中, 姬蜂总科新种数量最多, 约占全年发表新种总数的 2/3。云南、浙江、西藏、福建和辽宁等5个省级行政区为2020年中国膜翅目新种分布最多的省份，超过全年发表新种总数的 $1 / 2$ 。基于以上结果, 本文对膜翅目分类学现状和发展前景进行了讨论。

关键词: 细腰亚目; 广腰亚目; 分类学报告; 新分类单元分布

曹焕喜, 牛泽清, 唐璞, 李涛, 朱朝东 (2021) 中国膜翅目2020年新分类单元. 生物多样性, 29, 1026-1034. doi: 10.17520/biods.2021167. Cao HX, Niu ZQ, Tang P, Li T, Zhu CD (2021) New taxa and taxonomic changes of Hymenoptera from China in 2020. Biodiversity Science, 29, $1026-1034$. doi: 10.17520/biods.2021167.

\section{New taxa and taxonomic changes of Hymenoptera from China in 2020}

\author{
Huanxi Cao ${ }^{(\mathrm{D})}$, Zeqing Niu ${ }^{(\mathrm{D} 2}$, Pu Tang ${ }^{(\mathrm{D})}$, Tao $\mathrm{Li}^{(\mathrm{D})}{ }^{4}$, Chaodong Zhu ${ }^{(\mathrm{D} 2 *}$
}

1 National Animal Collection Resource Center, Institute of Zoology, Chinese Academy of Sciences, Beijing 100101

2 Key Laboratory of Zoological Systematics and Evolution, Institute of Zoology, Chinese Academy of Sciences, Beijing 100101

3 College of Agriculture and Biotechnology, Zhejiang University, Hangzhou 310058

4 General Station of Forest and Grassland Pest Management, National Forestry and Grassland Administration, Shenyang 110034

\section{ABSTRACT}

Aim: The aim of this study is to summarize the new taxa and taxonomic changes of Hymenoptera from China published in 2020.

Method: Through searching the available zoological databases and collecting data from Chinese hymenopterists, in 2020, 104 journal publications and 1 monograph related to the new taxa and taxonomic changes of Hymenoptera from China were obtained and analyzed.

Results: Based on these publications, 469 records of new taxa, new records and taxonomic changes of Hymenoptera from China were retrieved, including 4 new genera (1 extinct genus), 1 new subgenus, 321 new species (10 extinct species), 8 new combinations, 22 new synonyms, 1 newly recorded subfamily, 18 newly recorded genera, 93 newly recorded species, and 1 replaced name. In 11 records of them, 3 superfamilies, 3 families and 5 genera of extinct hymenopteran were involved. Another 458 records belong to 11 superfamilies, 29 families, 170 genera of extant Hymenoptera, of which 40 genera distributed in 6 families are only related to new distribution records or taxonomic changes. Among these 11 superfamilies, 9 superfamilies with new species published in 2020 include Symphyta:

收稿日期: 2021-04-30; 接受日期: 2021-08-09

基金项目: 国家自然科学基金委杰出青年基金(31625024)

* 通讯作者 Author for correspondence. E-mail: zhucd@ioz.ac.cn 
Tenthredinoidea (23 new species); Aculeata of Apocrita: Apoidea (29 new species), Chrysidoidea (2 new species), and Vespoidea (20 new species); Parasitica of Apocrita: Chalcidoidea (23 new species), Cynipoidea (12 new species), Ichneumonoidea (206 new species), Platygastroidea (4 new species), Trigonalyoidea (2 new species).

Conclusions: Among the 9 superfamilies, Ichneumonoidea had the largest number (206) of new species by covering about 2/3 of 321 new species published in 2020. Five provinces with more new species published in 2020 are Yunnan, Zhejiang, Xizang, Fujian, and Liaoning, covering about 1/2 of the 321 new species. Moreover, the current status and future development of Hymenoptera taxonomy were discussed here based on these results.

Key words: Apocrita; Symphyta; taxonomic report; distribution of new taxa

对生物进行分类是人类认识自然的基础。昆虫 是动物界最为繁盛的类群之一, 已发现的物种超过 100万种(Zhang, 2011), 是全球生物多样性的重要组 成部分, 更是生态系统服务不可或缺的重要基础。 昆虫分类学也是动物分类学的重要组成部分。

膜翅目目前是昆虫纲第三大目, 其已知物种数 量仅次于鞘翅目和鳞翅目(Aguiar et al, 2013)。根据 膜翅目年代最老的长节叶蜂科(Xyelidae, 在现生类 群里中文名常作棒蜂科) 化石记录, 目前膜翅目的 起源可追溯至三叠纪(Triassic), 距今约 224 个百万 年(Peters et al, 2017)。截至目前, 膜翅目已描述的现 生类群超过 15 万种, 已知绝灭物种 2,000种以上 (Aguiar et al, 2013)。据估测, 膜翅目实际物种数量 将有望超过100万种(Forbes et al, 2018)。仅仅基于已 知物种数量对膜翅目物种多样性进行的估计可能 与实际情况存在显著差异。综合考虑昆虫纲四大目 的分类学研究历史、受关注程度, 膜翅目分类的成 熟度和广泛性可能不如鞘翅目和鳞翅目。膜翅目寄 生类群的分类学研究相对匮乏, 其物种多样性可能 被严重低估(Forbes et al, 2018)。

膜翅目不仅物种多样性丰富, 其形态学和生物 学习性也复杂多样, 因此其功能多样性也存在着较 强的异质性。作为寄生者、捕食者、传粉者、分解 者和植食者, 膜翅目在陆地生态系统中发挥着至关 重要的作用, 其彰显的经济价值和重要性对人类而 言也意义非凡。基于系统学和形态学, 膜翅目学家 习惯将膜翅目分为广腰亚目(Symphyta)和细腰亚目 (Apocrita)。本文从理解系统学的简洁性和易操作性 角度出发, 依照传统仍然沿用广腰亚目和细腰亚目 的亚目分类系统。广腰亚目包括叶蜂类(sawflies)、 树蜂类(woodflies)和尾蜂(parasitic wood wasps)。除 尾蜂为寄生性外, 叶蜂和树蜂的幼虫均为植食性。 细腰亚目通常分为针尾部 (Aculeata) 和寄生部 (Parasitica); 针尾部又从行为和生态功能上被分为
蜜蜂 (bees)、蚂蚁 (ants) 和其他䖯刺蜂类 (stinging wasps), 而寄生部则包含了除针尾部之外的所有细 腰类群。然而, 并不是所有的寄生部都行寄生行为。 寄生部涵盖了膜翅目的大多数物种, 也正是膜翅目 分类学中最为薄弱的部分。

截至目前, 我国尚未有关于膜翅目分类学进展 的综述发表, 已知物种数量以及每年新分类单元的 发表情况也未有详细报告。为了整合零散的膜翅目 新分类单元发表记录, 本文基于数据库检索着重对 中国膜翅目2020年新分类单元发表情况进行了整 理和总结, 并依据文献记录对中国膜翅目近五年来 新种的发表趋势进行了简要分析。

\section{数据来源及分析}

\section{1 数据获取及整理}

在统计之初, 作者设计了表格向中国膜翅目分 类学者征集新分类单元(包括新种、新记录、新组合、 新异名、新名称等)的发表情况。共征集到8份统计 表格, 分别来自中国科学院动物研究所(姬小蜂科 Eulophidae和蜜蜂总科Apoidea)、浙江大学(茧蜂科 Braconidae)、国家林业和草原局森林和草原病虫害 防治总站(姬蜂科Ichneumonidae)、重庆师范大学(胡 蜂科 Vespidae)、华南农业大学 (缘腹细蜂科 Scelionidae)、中山大学 (缘腹细蜂科、肿腿蜂科 Bethylidae 、钩腹蜂科 Trigonalyidae 、螯蜂科 Dryinidae)、天津农业学院(跳小蜂科Encyrtidae)、甘 肃农业大学(化石姬蜂科类群)。在此基础上, 作者对 Zoological Record数据库和中国知网(www.cnki.net) 数据库根据关键词组合(Hymenoptera, new species, China)和限定发表年度(2020/2019/2018/2017/2016) 进行检索, 获取文献信息。经过检索和梳理, 最终 共获取于2020年正式发表的104篇相关期刊论文和 1部图书专著(《寄生叶蜂的姬蜂科天敌昆虫》)。据 此整理完成“中国膜翅目分类单元新增和变动目录 
2020” (附录1)。为方便和规范在中文中使用物种名, 避免出现一个拉丁学名对应多个不恰当中文种名 的现象, 该附录提供了物种的中文名。附录中列出 的中文名大部分由发表相应新分类单元的命名人 提供, 部分中文名由本文作者根据拉丁词源命名。

\section{2 数据分析}

将附录 1 中的数据转化为统计表格后导入 $\mathrm{R}$ 4.0.5 (R Core Team, 2021)进行分析。除基础包外, 使用到的R包还包括：ggplot2 (Villanueva \& Chen, 2019), dplyr (Wickham et al, 2021), stringr (Wickham, 2019), ggwordcloud (Le Pennec \& Slowikowski, 2019), tm (Feinerer et al, 2008)。

\section{结果}

\section{1 中国膜翅目2020年新分类单元基本情况}

2020年发表的中国膜翅目新分类单元中, 现生 膜翅目类群和绝灭类群新分类单元均有涉及, 根据 检索结果获取的新分类单元类别有: 新属, 新亚属, 新种, 新组合, 新记录亚科, 新记录属, 新记录种, 新异名, 替代名。分类单元新增及变动条目共 469 条, 包括: 4 个新属(其中 1 个绝灭属), 1 个新亚属, 321 个新种(其中 10 个绝灭种), 8 个新组合, 22 个新异 名, 1 个新记录亚科, 18 个新记录属, 93 个中国新记 录种, 1 个替代名。以上新分类单元发表情况包括细 腰亚目 435条, 广腰亚目34条; 除10个绝灭种外, 细 腰亚目新种 288 个(针尾部39个, 寄生部249个), 广 腰亚目新种23个。

发表的绝灭类群包括: 广腰亚目1新属9新种和 细腰亚目1新种。发表的现生类群包括: 广腰亚目 2 总科 (叶蜂总科 Tenthredinoidea 和树蜂总科 Siricoidea); 细腰亚目针尾部3总科(蜜蜂总科、青蜂 总科Chrysidoidea和胡蜂总科 Vespoidea), 寄生部6 总科(小蜂总科Chalcidoidea, 瘞蜂总科Cynipoidea, 姬蜂总科 Ichneumonoidea, 广腹细蜂总科 Platygastroidea, 细蜂总科Proctotrupoidea, 钩腹蜂 总科Trigonalyoidea)。上述涉及发表新种的 9 个总科 (树蜂总科和细蜂总科未涉及新种发表)共涵盖23科 129属。其中, 中国姬蜂总科2020年发表新种数量居 首, 多达206个, 约占全年发表的中国现生膜翅目 新种数量的 $66.2 \%$ 。

2020年发表的中国膜翅目新记录均为现生类 群, 包括 1 个新记录亚科(隶属姬蜂科)、18个新记录
属(茧蜂科4属、姬蜂科14属)和93个新记录种(隶属7 总科12科47属) (表1)。2020年提出的中国膜翅目 22 个新异名均为现生类群, 隶属6总科 9 科 13 属。2020 年发表的中国膜翅目8新组合均为现生类群, 隶属2 科3属。2020年发表的中国膜翅目1个替代名隶属切 叶蜂科(Megachilidae)切叶蜂属(Megachile)。

\section{2 新种和新记录种的省级分布情况}

2020年发表的中国膜翅目新种的分布地涉及 33 个省级行政区，本年度没有发现膜翅目新种的省 级行政区有 1 个, 为上海市。新种发现数量超过 20 个的省级行政区有 6 个, 包括云南、浙江、西藏、福 建、辽宁和四川。新种发现数量在10-20个之间的 省级行政区有12个，包括江西、贵州、台湾、广西、 北京、山东、广东、湖南、吉林、海南、陕西和甘 肃。新种发现数量在 10 个以下的省级行政区有 15 个, 包括宁夏、湖北、香港、新疆、河北、黑龙江、河 南、青海、安徽、江苏、内蒙古、天津、山西、重 庆和澳门(图1)。

2020年发表的膜翅目中国新记录种的分布地 记录有25个省级行政区，本年度没有发现膜翅目中 国新记录种的省级行政区有 9 个(安徽、甘肃、香港、 湖北、江苏、澳门、青海、上海、天津)。其中, 新 记录种数量超过 20 个的只有辽宁省, 《寄生叶蜂的 姬蜂科天敌昆虫》(盛茂领等, 2020)贡献了 25 个中国 新记录种(隶属姬蜂科)。中国新记录种数量在10-20 个的省级行政区有 7 个, 包括云南、吉林、福建、浙 江、广东、海南。中国新记录种数量在 2 个以上 10 个以下的省级单位有北京、贵州、宁夏和内蒙古。 湖南、江西、山东、四川、台湾分别有 2 个新记录 种。重庆、河北、河南、陕西、山西、西藏分别只 有1个新记录种(图1)。

\subsection{0 年发表中国膜翅目新分类单元的学者}

2020年，发表 10 个绝灭种的 2 篇期刊论文共涉 及13位论文作者和12个新种命名人, 其中俄罗斯科 学院A. A. Borissiak古生物学研究所的Dmitry S. Kopylov和Alexander P. Rasnitsyn、中国科学院南京 地质古生物研究所的张海春、曲阜师范大学的张琦 共同描述了 8 个绝灭种。

关于现生膜翅目类群, 发表 81 个中国姬蜂科新 种的中文图书专著《寄生叶蜂的姬蜂科天敌昆虫》 的作者有3位，同时也是 81 个新种的命名人，均来 自国家林业和草原局森林和草原病虫害防治总 
表12020年中国膜翅目各科新种和新记录种数量(不含绝灭种)

Table 1 The number of new species and new records of Hymenoptera from China at the family level in 2020 (without extinct species)

\begin{tabular}{|c|c|c|c|c|c|}
\hline $\begin{array}{l}\text { 亚目 } \\
\text { Suborder }\end{array}$ & $\begin{array}{l}\text { 总科 } \\
\text { Superfamily }\end{array}$ & $\begin{array}{l}\text { 中文科名 } \\
\text { Family name in Chinese }\end{array}$ & $\begin{array}{l}\text { 科 } \\
\text { Family }\end{array}$ & $\begin{array}{l}\text { 新种数量 } \\
\text { No. of new species }\end{array}$ & $\begin{array}{l}\text { 新记录种数量 } \\
\text { No. of new records }\end{array}$ \\
\hline \multirow{9}{*}{$\begin{array}{l}\text { 细腰亚目针尾部 } \\
\text { Apocrita (Aculeata) }\end{array}$} & \multirow[t]{4}{*}{ 蜜蜂总科 Apoidea } & 方头泥蜂科 & Crabronidae & 7 & 3 \\
\hline & & 隧蜂科 & Halictidae & 4 & 2 \\
\hline & & 切叶蜂科 & Megachilidae & 5 & 0 \\
\hline & & 三室泥蜂科 & Psenidae & 3 & 0 \\
\hline & \multirow[t]{2}{*}{ 青蜂总科 Chrysidoidea } & 肿腿蜂科 & Bethylidae & 2 & 0 \\
\hline & & 螯蜂科 & Dryinidae & 0 & 1 \\
\hline & \multirow[t]{3}{*}{ 胡蜂总科 Vespoidea } & 蚁科 & Formicidae & 12 & 0 \\
\hline & & 蚁蜂科 & Mutillidae & 2 & 1 \\
\hline & & 胡蜂科 & Vespidae & 6 & 1 \\
\hline \multirow{12}{*}{$\begin{array}{l}\text { 细腰亚目寄生部 } \\
\text { Apocrita (Parasitica) }\end{array}$} & \multirow[t]{6}{*}{ 小蜂总科 Chalcidoidea } & 蚜小蜂科 & Aphilinidae & 1 & 2 \\
\hline & & 跳小蜂科 & Encyrtidae & 6 & 1 \\
\hline & & 姬小蜂科 & Eulophidae & 4 & 1 \\
\hline & & 旋小蜂科 & Eupelmidae & 2 & 0 \\
\hline & & 姃小蜂科 & Mymaridae & 7 & 0 \\
\hline & & 金小蜂科 & Pteromalidae & 3 & 0 \\
\hline & \multirow[t]{2}{*}{ 㾉蜂总科 Cynipoidea } & 㾉蜂科 & Cynipidae & 11 & 0 \\
\hline & & 环腹㾉蜂科 & Figitidae & 1 & 0 \\
\hline & \multirow[t]{2}{*}{ 姬蜂总科 Ichneumonoidea } & 茧蜂科 & Braconidae & 107 & 16 \\
\hline & & 姬蜂科 & Ichneumonidae & 99 & 49 \\
\hline & \multicolumn{2}{|c|}{ 广腹细蜂总科 Platygastroidea 缘腹细蜂科 } & Scelionidae & 4 & 15 \\
\hline & 钩腹蜂总科 Trigonalyoidea & 钩腹蜂科 & Trigonalyidae & 2 & 0 \\
\hline \multirow{4}{*}{$\begin{array}{l}\text { 广腰亚目 } \\
\text { Symphyta }\end{array}$} & \multirow[t]{3}{*}{ 叶蜂总科 Tenthredinoidea } & 三节叶蜂科 & Argidae & 2 & 0 \\
\hline & & 锤角叶蜂科 & Cimbicidae & 3 & 0 \\
\hline & & 叶蜂科 & Tenthredinidae & 18 & 0 \\
\hline & 树蜂总科 Siricoidea & 项蜂科 & Xiphydriidae & 0 & 1 \\
\hline 合计 Total & 10 & 25 & 25 & 311 & 93 \\
\hline
\end{tabular}

站; 86篇期刊论文共计发表了230个中国现生膜翅 目新种, 涉及论文作者209位、新种命名人108位(图 2): 86 篇期刊论文作者总数量为 341 (平均每篇论文 4 位作者); 230 个新种命名人总数量为 530 (平均每 个新种的命名人超过 2 人)。描述中国现生膜翅目新 种数量居前的单位有国家林业和草原局森林和草 原病虫害防治总站、浙江大学、江西师范大学、华 东药用植物园、中国科学院动物研究所、香港大学、 云南农业大学、重庆师范大学、新疆大学、中山大 学等(表2)。描述 5 个及以上新种的学者有23位, 其中 描述物种数量最多的团队为国家林业和草原局森 林和草原病虫害防治总站的盛茂领团队、浙江大学 陈学新团队, 分别描述新种多达 93 和 92 个, 且描述
类群均属于姬蜂总科。其次为江西师范大学魏美才 团队(叶蜂总科 23 个新种)、中国科学院动物研究所 朱朝东团队 (蜜蜂总科和姬小蜂科 9 个新种)、香港大 学的Benoit Guénard团队(蚁科和三室泥蜂科 8 个新 种)和云南农业大学李强团队(方头泥蜂科和泥蜂科 7 个新种)。

\subsection{0 年发表中国膜翅目新种论文的期刊}

2020年, 收录中国膜翅目昆虫新分类单元的论 文或图书专著共计105篇(部), 涉及的期刊或出版社 共计 36个: SCI期刊21个, 其他期刊14个, 中国出版 社 1 个; 收录绝灭类群的期刊 2 个, 其余 33 个期刊均 收录现生类群。收录绝灭类群的期刊(Alcheringa: An Australasian Journal of Palaeontology) (2篇)和 


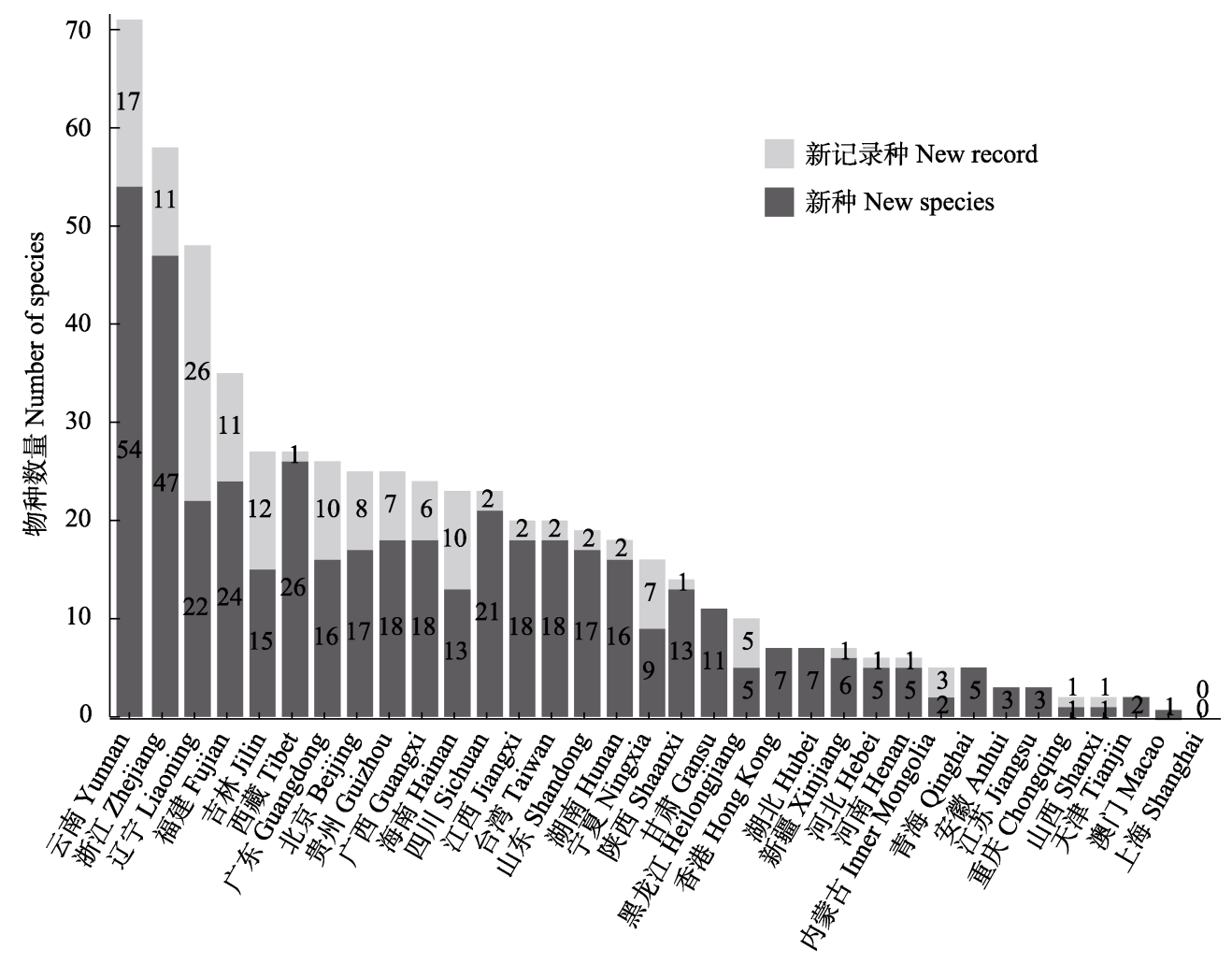

图12020年发表的中国膜翅目新种和新记录种在各省级行政区分布情况(不含绝灭种)

Fig. 1 The number of new species and new record species of Hymenoptera from China in 2020 in each province (without extinct taxa)

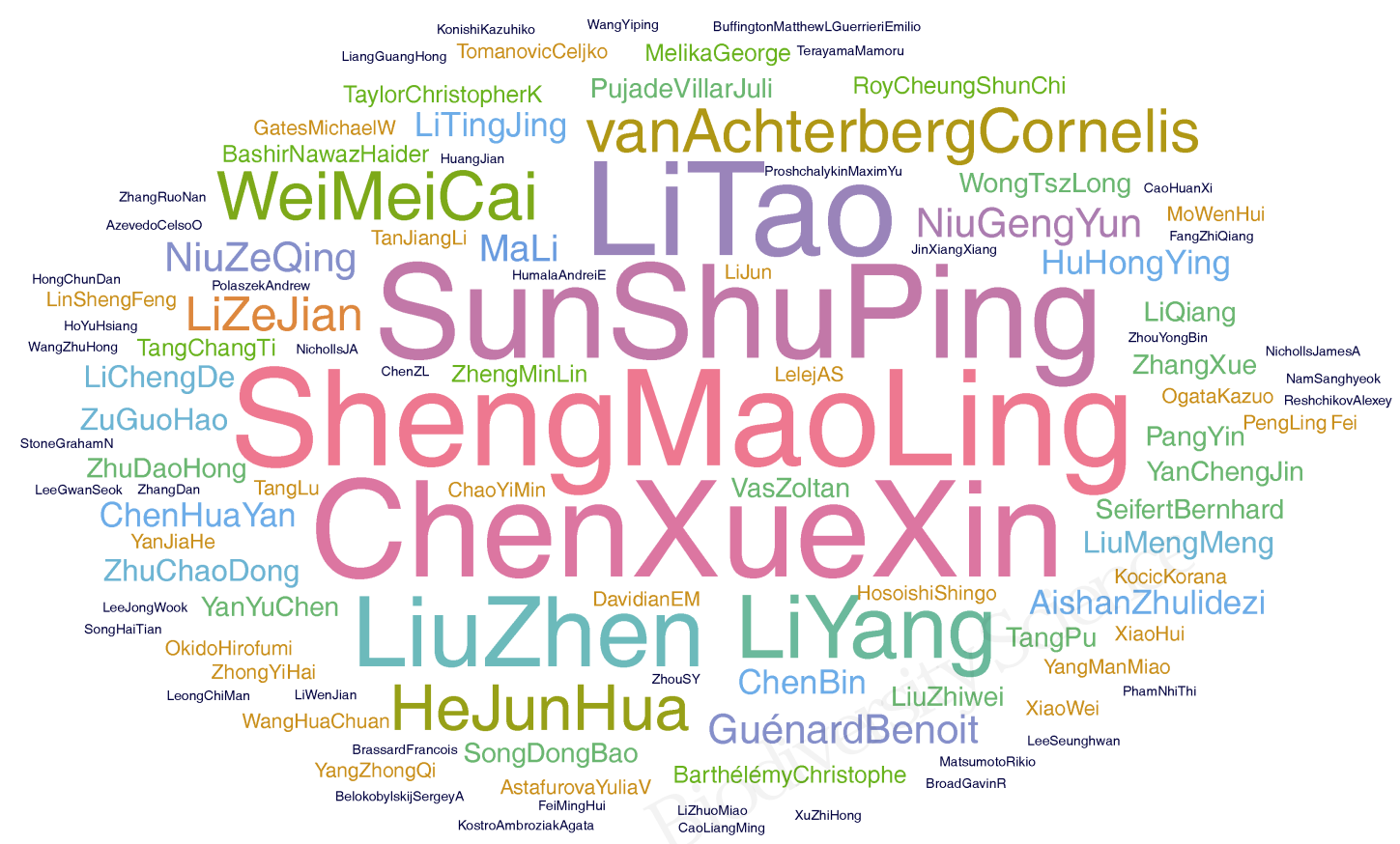

图2 基于2020年命名的中国膜翅目新种数量的命名人名字词云(不含绝灭种)

Fig. 2 A word cloud for the name of authors based on the number of new species of Hymenoptera from China they described in 2020 (without extinct taxa) 
表2 2020年发表5个及以上中国膜翅目新种的学者(不含绝灭种)

Table 2 Authors published 5 and more new species of Hymenoptera from China in 2020 (without extinct species)

\begin{tabular}{|c|c|c|}
\hline $\begin{array}{l}\text { 命名人 } \\
\text { Author }\end{array}$ & $\begin{array}{l}\text { 命名新种数量 } \\
\text { No. of new species described }\end{array}$ & $\begin{array}{l}\text { 单位 } \\
\text { Affiliation }\end{array}$ \\
\hline 盛茂领 Maoling Sheng & 93 & $\begin{array}{l}\text { 国家林业和草原局森林和草原病虫害防治总站 General Station of Forest and } \\
\text { Grassland Pest Management, National Forestry and Grassland Administration }\end{array}$ \\
\hline 陈学新 Xuexin Chen & 92 & 浙江大学 Zhejiang University \\
\hline 孙淑萍 Shuping Sun & 90 & $\begin{array}{l}\text { 国家林业和草原局森林和草原病虫害防治总站 General Station of Forest and } \\
\text { Grassland Pest Management, National Forestry and Grassland Administration }\end{array}$ \\
\hline 李涛 Tao Li & 88 & $\begin{array}{l}\text { 国家林业和草原局森林和草原病虫害防治总站 General Station of Forest and } \\
\text { Grassland Pest Management, National Forestry and Grassland Administration }\end{array}$ \\
\hline 刘珍 Zhen Liu & 48 & 浙江大学 Zhejiang University \\
\hline 李杨 Yang Li & 40 & 浙江大学 Zhejiang University \\
\hline 魏美才 Meicai Wei & 23 & 江西师范大学 Jiangxi Normal University \\
\hline 何俊华 Junhua He & 22 & 浙江大学 Zhejiang University \\
\hline Cornelis van Achterberg & 20 & 浙江大学、西北大学 Zhejiang University, Northwest University \\
\hline 李泽建 Zejian Li & 11 & $\begin{array}{l}\text { 华东药用植物园科研管理中心 Scientific Research and Management Center of } \\
\text { East China Pharmaceutical Botanical Garden }\end{array}$ \\
\hline 牛耕耘 Gengyun Niu & 9 & 江西师范大学 Jiangxi Normal University \\
\hline Benoit Guénard & 8 & 香港大学 Hong Kong University \\
\hline 牛泽清 Zeqing Niu & 8 & 中国科学院动物研究所 Institute of Zoology, Chinese Academy of Sciences \\
\hline 马丽 Li Ma & 7 & 云南农业大学 Yunnan Agricultural University \\
\hline 陈斌 Bin Chen & 6 & 重庆师范大学 Chongqing Normal University \\
\hline 李廷景 Tingjing Li & 6 & 重庆师范大学 Chongqing Normal University \\
\hline 陈华燕 Huayan Chen & 6 & 中山大学 Sun Yat-sen University \\
\hline 朱丽得孜·艾山 Aishan Zhulidezi & 6 & 新疆大学 Xinjiang University \\
\hline 胡红英 Hongying Hu & 6 & 新疆大学 Xinjiang University \\
\hline 刘萌萌 Mengmeng Liu & 5 & 丽水学院 Lishui University \\
\hline 李承德 Chengde Li & 5 & 东北林业大学 Northeast Forestry University \\
\hline 祖国浩 Guohao Zu & 5 & 天津农学院 Tianjin Agricultural University \\
\hline 朱朝东 Chaodong Zhu & 5 & 中国科学院动物研究所 Institute of Zoology, Chinese Academy of Sciences \\
\hline
\end{tabular}

Journal of Systematic Palaeontology (1篇)共发表3篇 论文，涉及 1 个新属和 10 个新种。

收录现生类群的33个期刊共发表102篇论文: 中文论文7篇，俄语论文 1 篇，其余94篇均为英文论 文; SCI论文73篇, 其他论文29篇; 涉及新种的论文 共计86篇。尽管2020年涉及中国现生膜翅目昆虫新 分类单元的期刊有 33 个, 但其中 22 个期刊均只收录 了1篇论文, 其中的6篇论文未涉及新种发表、7篇论 文仅发表了 1 个新种。在这 33 个期刊中, 分类学领域 的著名期刊Zootaxa深受膜翅目学者欢迎, 无论是 发表的论文数量还是新种数量, 均高居榜首, 共计 26篇论文、114个新种(图3), 单篇平均发表新种超过 4 个。在这26篇Zootaxa 论文中: 其中1篇发表新种数 量高达48个(线茧蜂属Apanteles Foerster), 其次为 11 个(茧蜂亚科Braconinae的6属), 均来自浙江大学陈
学新团队; 有 10 篇仅发表了 1 个新种; 国外学者独 立发表有关中国膜翅目新分类单元的论文3篇。在 发表论文数量上, 居于次席的为ZooKeys, 共计16 篇论文、17个新种，单篇平均发表新种 1 个左右(图 3)。在发表新种数量上, 居于次席的为《昆虫分类 学报》, 共计9篇论文、19个新种, 单篇平均发表新 种 2 个左右。另外, 中文图书专著《寄生叶蜂的姬蜂 科天敌昆虫》一书贡献了中国膜翅目2020年新分类 单元143条, 包括13新记录属、81新种和48新记录种。

\section{5 近五年中国膜翅目昆虫新种发表情况}

2016-2020年，每年平均发表约88篇有关中国 膜翅目新种的期刊论文, 新种平均约193个(现生物 种185个, 绝灭种 8 个)。2016-2019年, 每年发表的中 国膜翅目新种数量以及期刊论文相对稳定, 新种数 量略有下降，但不显著(图4)。2016-2019年，每年平 


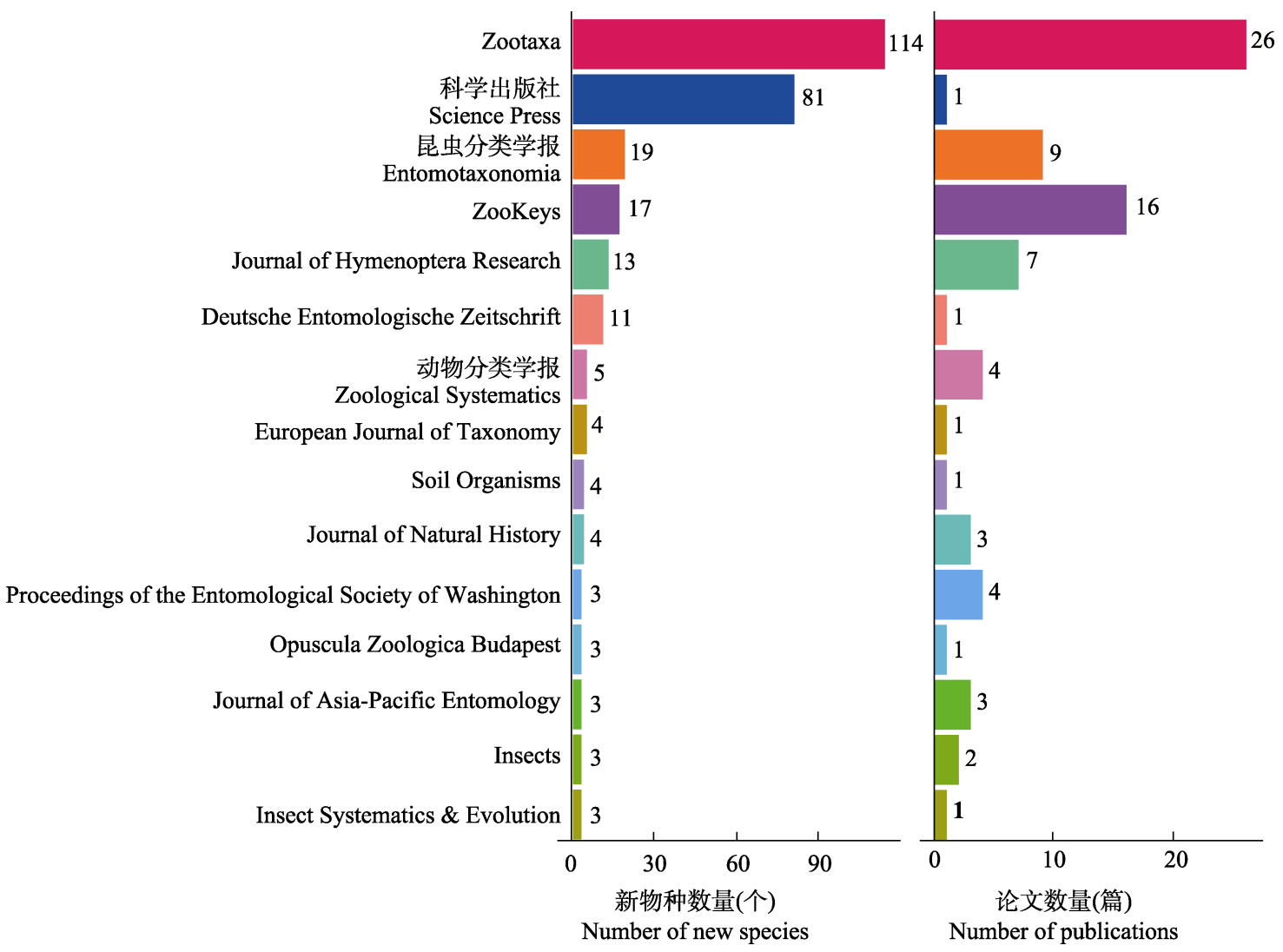

图32020年发表中国膜翅目新种数量超过 2 个的期刊/出版社发表的新种及论文数量(不含绝灭种)

Fig. 3 The number of new species of Hymenoptera from China and related references published by the journals/publishers that published more than 2 new species of Hymenoptera in China in 2020 (without extinct taxa)

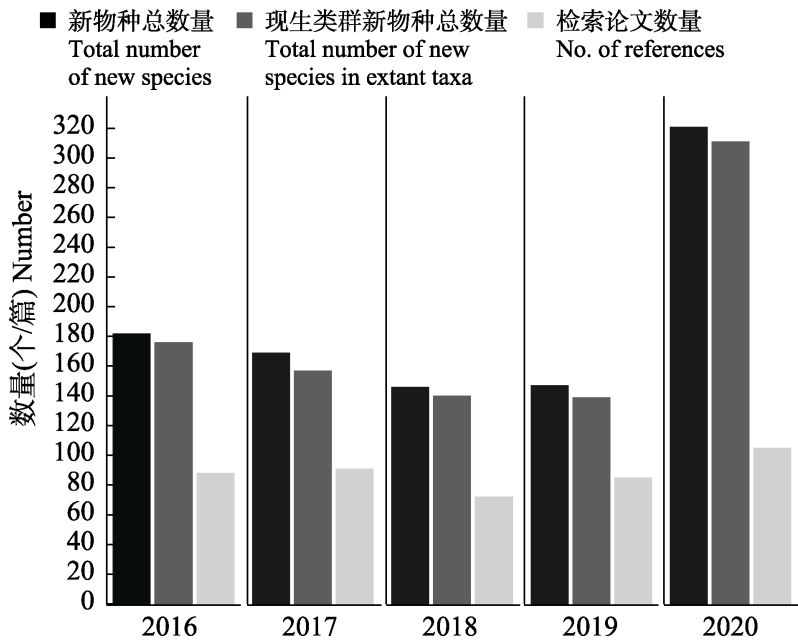

图4 2016年以来每年发表的中国膜翅目新种数量

Fig. 4 The number of new species of Hymenoptera from China described each year since 2016

均发表约84篇有关中国膜翅目新种的期刊论文和 153 个新种(现生种 145 个, 绝灭种 8 个)。相较于前 4 个年度, 2020年发表的中国膜翅目新种无论是在文 献数量上还是新种数量上都有了显著提升(图4)。即
使不考虑《寄生叶蜂的姬蜂科天敌昆虫》发表的 81 个新种，2020年发表的中国膜翅目新种也高达240 个, 显著多于本次统计结果中前 4 个年度中最高的 182个(2016年度)。

\section{3 讨论}

2020年发表的中国膜翅目现生类群新种(以下 讨论均指中国膜翅目现生类群)数量多达 311 个, 为 2016年以来发表数量最多的年份。尽管如此, 新种 所属类群的结构不够均衡, 所属总科数量接近膜翅 目总科数量的 $1 / 3$ (为 $9 / 23$ ), 但所属科数量约占膜翅 目科总数量的 $1 / 4$ (为23/94) (现生膜翅目总科及科 的总数量参考Aguiar et al, 2013)。姬蜂总科新种数 量约占全年发表新物数量的 $2 / 3$, 其次为小蜂总科 和叶蜂总科(均为 23 个), 仅占姬蜂总科新种数量的 $1 / 10$ 左右。即使不考虑《寄生叶蜂的姬蜂科天敌昆 虫》中的 81 个新种, 小蜂总科和叶蜂总科发表的新 种数量也不足姬蜂总科新种数量的 $1 / 5$ 。这说明, 2020年姬蜂总科分类学研究在中国膜翅目研究中 
独占鳌头。这种不均衡的结构同时体现在2020年中 国膜翅目学者命名新种的数量上(图2)。

新种发表并不是也不能作为衡量分类学进展 的唯一标准。因此, 仅依据新种发表数据是否能够 说明中国姬蜂总科分类学进展强于其他膜翅目类 群, 仍然需要更多数据支持。同时, 这也是需要每 一位中国膜翅目分类学者思考的问题。但这从一定 程度上说明, 绝大多数中国膜翅目类群分类学的发 展仍然需要各个膜翅目研究团队以及有关部门向 基础分类学投入更多的关注和支持。分类学人才梯 队形成且稳定以及标本采集、收集和研究力度较大, 可能是浙江(浙江大学所在地)和辽宁 (国家林业和 草原局森林和草原病虫害防治总站所在地)发表新 种数量居前的原因之一。云南、西藏和福建可能凭 借其自然环境优势, 吸引膜翅目学者对这些省区的 标本采集和收集产生一定的偏好性。

在期刊(略去图书专著)选择上, 著名分类学期 刊Zootaxa仍是中国膜翅目分类学者发表分类学成 果的主阵地, 且单篇平均发表新种数量超过另外一 个著名的分类学期刊ZooKeys。究其原因, 可能是期 刊发表政策存在差异, 如审稿周期和出版费用。 Zootaxa 的审稿周期相对较长, 但如果不选择开放 获取, 无需支付高额出版费用; ZooKeys的审稿周期 相对较短, 论文全部开放获取, 但需支付高昂的出 版费用。此外, 根据作者投稿经验, Zootaxa并不鼓 励单个新种的发表, 综合性的分类综述或者厘定工 作更受欢迎，而ZooKeys仍可接收单个新种的论文。 在本文统计的26篇Zootaxa论文中, 可以划归到分 类学综述或者厘定工作中的论文数量比例可能不 足 $1 / 2$ 。涉及现生膜翅目新分类单元的 14 个非SCI期 刊中, 6 个期刊出版地为中国, 共计 19 篇论文, 约占 2020年全年论文数量(102篇)的18.6\%; 其中发表中 国膜翅目新种数量最多的期刊为《昆虫分类学报》, 但发表类群主要为叶蜂总科类群, 其次为《动物分 类学报》。

需要指出的是, 本文有关2016年以来每年发表 的中国膜翅目新种数量的趋势, 可能并不能体现近 五年来的实际情况。尽管笔者尽可能确保 2016-2019年的新种发表数量检索和统计的全面性, 但并不能保证如2020年度检索和统计结果般精确 和准确, 仅提供一个概览。
致谢: 感谢《生物多样性》主编马克平研究员的邀 请, 副主编黄晓磊教授等前期讨论和策划。浙江大 学陈学新教授、国家林业和草原局森林和草原病虫 害防治总站盛茂领研究员、中国林业科学院杨忠岐 研究员、江西师范大学魏美才教授、首都师范大学 任东教授、云南农业大学李强教授、重庆师范大学 李廷景教授、新疆大学胡红英教授、华南农业大学 刘经贤副研究员、中山大学陈华燕博士和天津农学 院祖国浩博士等团队及时提供了相关信息。感谢相 关类群专家为本文附录中的新物种提供了中文物 种名。本文责任编委和两位评审专家对本文提出了 宝贵意见和建议, 在此一并致谢。

\section{ORCID}

曹焕喜 (D) https://orcid.org/0000-0002-5827-2776

牛泽清 (D) https://orcid.org/0000-0002-0964-0317

唐璞 (D) https://orcid.org/0000-0003-2296-9544

李涛 (D) https://orcid.org/0000-0001-6758-3472

朱朝东 (D) https://orcid.org/0000-0002-9347-3178

\section{参考文献}

Aguiar AP, Deans AR, Engel MS, Forshage M, Huber JT, Jennings JT, Johnson NF, Lelej AS, Longino JT, Lohrmann V, Miko I, Ohl M, Rasmussen C, Taeger A, Yu DSK (2013) Order Hymenoptera. Zootaxa, 3703, 51-62.

Feinerer I, Hornik K, Meyer D (2008) Text Mining Infrastructure in R. Journal of Statistical Software, 25(5), $1-54$.

Forbes AA, Bagley RK, Beer MA, Hippee AC, Widmayer HA (2018) Quantifying the unquantifiable: Why Hymenoptera, not Coleoptera, is the most speciose animal order. BMC Ecology, 18, 21.

Le Pennec E, Slowikowski K (2019) ggwordcloud: A Word Cloud Geom for 'ggplot2'. R package version 0.5.0. https:// CRAN.R-project.org/package=ggwordcloud. (accessed on 2021-04-17)

Peters RS, Krogmann L, Mayer C, Donath A, Gunkel S, Meusemann K, Kozlov A, Podsiadlowski L, Petersen M, Lanfear R, Diez PA, Heraty J, Kjer KM, Klopfstein S, Meier R, Polidori C, Schmitt T, Liu SL, Zhou X, Wappler T, Rust J, Misof B, Niehuis O (2017) Evolutionary history of the Hymenoptera. Current Biology, 27, 1013-1018.

R Core Team (2021) R: A Language and Environment for Statistical Computing. R Foundation for Statistical Computing, Vienna, Austria. https://www.R-project.org/. (accessed on 2021-04-17)

Sheng ML, Sun SP, Li T (2020) Ichneumonids parasitizing sawflies in China (Hymenoptera, Ichneumonidae). Science Press, Beijing. (in Chinese with English abstract) [盛茂领, 
孙淑萍, 李涛 (2020) 寄生叶蜂的姬蜂科天敌昆虫. 科学 出版社, 北京.]

Villanueva RAM, Chen ZJ (2019) ggplot2: Elegant Graphics for Data Analysis (2nd edn.). Measurement: Interdisciplinary Research and Perspectives, 17, 160-167.

Wickham H (2019) stringr: Simple, Consistent Wrappers for Common String Operations. $\mathrm{R}$ package version 1.4.0. https://CRAN.R-project.org/package=stringr. (accessed on 2021-04-17)
Wickham H, François R, Henry L, Müller K (2021) dplyr: A Grammar of Data Manipulation. R package version 1.0.5. https:/CRAN.R-project.org/package=dplyr. (accessed on 2021-04-17)

Zhang ZQ (2011) Animal biodiversity: An introduction to higher-level classification and taxonomic richness. Zootaxa, 3148, 7-12.

(责任编委: 周欣 责任编辑: 间文杰)

\section{附录 Supplementary Material}

附录1 中国膜翅目2020年分类单元新增和变动目录

Appendix 1 Annual list of new taxa and taxonomic changes of Hymenoptera from China in 2020

https://www.biodiversity-science.net/fileup/PDF/2021167-1.pdf

\section{•新书评介・}

\section{《洞穴生物学》评介}

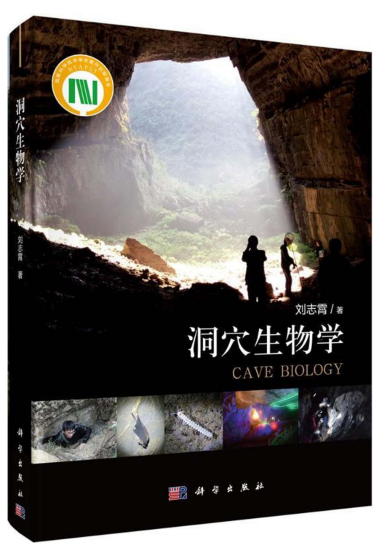

《洞穴生物学》(作者: 刘志霄教授)近期已由科学出版社出版, 这是我国第一部有关洞穴 生物多样性及其保护的专著。该书内容丰富, 从洞穴学基础、洞穴生物学的基本概念和发展 历程, 到洞穴生态系统的特点、洞穴生物多样性的构成, 以及洞穴考古、洞穴旅游和洞穴资 源保护等方面均有较为系统而新颖的论述, 具有重要的学习和研究参考价值, 因此特向同 行、学生和公众推荐。

在书中, 作者构建了洞穴生物学的基本框架和概念体系, 展现了洞穴生物多样性的基 本面貌, 反映了国内外洞穴生物学的研究进展、发展趋势和前景。该书得到国家科学技术学 术著作出版基金的立项资助，并由我国著名的生物学家洪德元院士、魏辅文院士以及美国著 名的洞穴生物学家Aldemaro Romero教授分别作序。

2021年是国际洞穴联合会(UIS)倡导的洞穴与喀斯特年(IYCK021)。10月, 联合国《生物 多样性公约》第十五次缔约方大会(COP15)将在昆明召开。相信该书的出版, 将会有助于推 动我国洞穴生物学, 特别是洞穴生物多样性保护事业的发展。

(华南农业大学 田明义) 


\section{附录}

\section{中国膜翅目分类单元新增和变动目录 2020 年}

\section{Annual List of New Taxa and Taxonomic Changes of Hymenoptera from China 2020}

曹焕喜, 牛泽清, 唐璞, 李涛, 朱朝东

Cao Huan-Xi, Niu Ze-Qing, Tang Pu, Li Tao, Zhu Chao-Dong 


\section{Apocrita 细腰亚目}

\section{Aculeata 针尾部}

\section{Apoidea 蜜蜂总科}

\section{Crabronidae 方头泥蜂科}

Carinostigmus frontirugatus Bashir \& Ma, 2020

皱额隆痣短柄泥蜂 [New Species]

Holotype $q$ (YNAU).

Distribution: CHINA (Guizhou, Yunnan).

Bashir, Ma \& Li (2020) ZooKeys 987: 115-134.

Carinostigmus latidentatus Bashir \& Ma, 2020 侧

齿隆疬短柄泥蜂 [New Species]

Holotype $q$ (YNAU).

Distribution: CHINA (Yunnan, Fujian, Hainan).

Bashir, Ma \& Li (2020) ZooKeys 987: 115-134.

Carinostigmus vesulcatus Bashir \& Ma, 2020 缺 沟隆痣短柄泥蜂 [New Species]

Holotype $q$ (YNAU).

Distribution: CHINA (Yunnan).

Bashir, Ma \& Li (2020) ZooKeys 987: 115-134.

Polemistus clypeisinuatus $\mathrm{Ma} \& \mathrm{Li}, 2020$ 波唇狭

额短柄泥蜂 [New Species]

Holotype $q$ (YNAU).

Distribution: CHINA (Zhejiang).

Ma \& Li (2020) Zootaxa 4852(4): 475-484.

Polemistus divaricatus $\mathrm{Ma} \& \mathrm{Li}, 2020$ 二叉狭额

短柄泥蜂 [New Species]

Holotype $\delta$ (YNAU).

Distribution: CHINA (Yunnan).

Ma \& Li (2020) Zootaxa 4852(4): 475-484.

Polemistus gutianus Ma \& Li, 2020 古田山狭额 短柄泥蜂 [New Species]
Holotype $q$ (YNAU).

Distribution: CHINA (Zhejiang).

Ma \& Li (2020) Zootaxa 4852(4): 475-484.

Polemistus pediflavidus $\mathrm{Ma} \& \mathrm{Li}, 2020$ 褐足狭额 短柄泥蜂 [New Species]

Holotype $q$ (YNAU).

Distribution: CHINA (Yunnan).

Ma \& Li (2020) Zootaxa 4852(4): 475-484.

Belomicrus kaszabi Tsuneki, 1972 卡氏小刺泥蜂

[New Record]

Distribution: CHINA (Inner Mongolia); Mongolia.

Li \& Ma (2020) Southwest China Journal of Agricultural Sciences 33(6): 1332-1336.

Belomicrus shatalkini Antropov, 1995 滑胸小刺 泥蜂 [New Record]

Distribution: CHINA (Inner Mongolia); Turkmenistan.

Li \& Ma (2020) Southwest China Journal of Agricultural Sciences 33(6): 1332-1336.

Carinostigmus palawanensis (Tsuneki), 1976 巴 拉望隆疬短柄泥蜂 [New Record]

Distribution: CHINA (Yunnan); Philippines.

Bashir, Ma \& Li (2020) ZooKeys 987: 115-134.

\section{Halictidae 隧蜂科}

Lasioglossum (Dialictus) submandibulare Niu, 2020 拟大颚淡脉隧蜂 [New Species]

Holotype $q$ (IZCAS).

Distribution: CHINA (Sichuan).

Niu, Zhang \& Zhu (2020) Zoological Systematics 45(1): 50-58. 
Nomia (Gnathonomia) fusciventris Zhang \& Niu, 2020 棕腹彩带蜂 [New Species]

Holotype $\widehat{\jmath}$ (IZCAS).

Distribution: CHINA (Fujian).

Zhang et al. (2020) Zootaxa 4768(1): 076-094.

Sphecodes holgeri Astafurova \& Proshchalykin, 2020 霍氏红腹蜂 [New Species]

Holotype $q$ (IZCAS).

Distribution: CHINA (Yunnan).

Astafurova et al. (2020) Journal of Hymenoptera

Research 79: 145-162.

Sphecodes tibeticus Astafurova \& Niu, 2020 西藏

红腹蜂 [New Species]

Holotype $ð$ (IZCAS).

Distribution: CHINA (Tibet).

Astafurova et al. (2020) Journal of Hymenoptera

Research 79: 145-162.

Nomia aurata Bingham, 1897 金花彩带蜂 [New Record]

Distribution: CHINA (Yunnan); South Asia.

Zhang et al. (2020) Zootaxa 4768(1): 076-094.

Nomia wahisi Pauly, 2009 瓦氏彩带蜂 [New Record]

Distribution: CHINA (Hainan); Laos, Thailand.

Zhang et al. (2020) Zootaxa 4768(1): 076-094.

\section{Megachilidae 切叶蜂科}

Anthidium (Anthidium) pseudomontanum Niu \& Zhu, 2020 拟山黄斑蜂 [New Species]

Holotype $q$ (IZCAS).

Distribution: CHINA (Xinjiang).

Niu et al. (2020) Zootaxa 4867(1): 001-067.
Anthidium (Anthidium) pseudophilorum Niu \& Zhu, 2020 拟红跗黄斑蜂 [New Species]

Holotype $\widehat{\jmath}$ (IZCAS).

Distribution: CHINA (Xinjiang, Qinghai, Tibet).

Niu et al. (2020) Zootaxa 4867(1): 001-067.

Anthidium (Anthidium) tasitiense Niu \& Zhu, 2020 塔斯提黄斑蜂 [New Species]

Holotype $\widehat{\jmath}$ (IZCAS).

Distribution: CHINA (Xinjiang).

Niu et al. (2020) Zootaxa 4867(1): 001-067.

Anthidium (Anthidium) xuezhongi Niu \& Zhu, 2020 学忠黄斑蜂 [New Species]

Holotype $\overparen{\jmath}$ (IZCAS).

Distribution: CHINA (Xinjiang, Tibet).

Niu et al. (2020) Zootaxa 4867(1): 001-067.

Anthidium (Proanthidium) qingtaoi Niu \& Zhu, 2020 清涛黄斑蜂 [New Species]

Holotype $\widehat{\jmath}$ (IZCAS).

Distribution: CHINA (Tibet).

Niu et al. (2020) Zootaxa 4867(1): 001-067.

Megachile (Callomegachile) parornata Chatthanabun, Warrit and Ascher, 2020 拟细点 切叶蜂 [Replaced Name]

Junior primary homonym: Megachile gigas $\mathrm{Wu}$, 2005 大切叶蜂

Distribution: CHINA (Yunnan); Vietnam, Thailand.

Chatthanabun et al. (2020) ZooKeys 997: 95-114.

Anthidium (Anthidium) florentinum (Fabricius, $1775)$ 花黄斑蜂 
曹焕喜, 牛泽清, 唐㒒, 李涛, 朱朝东 (2021) 中国膜翅目 2020 年新分类单元. 生物多样性, 29, 1026-1034. https://www.biodiversity-science.net/CN/10.17520/biods.2021167

Synonym: Anthidium (Proanthidium)

helianthinum $\mathrm{Wu}, 2004$ 向日葵黄斑蜂 [New Synonym]

Distribution: CHINA (Jiangsu, Inner Mongolia, Xinjiang, Gansu, Sichuan); widely distributed around the world.

Niu et al. (2020) Zootaxa 4867(1): 001-067.

Anthidium (Anthidium) kashmirense

Mavromoustakis, 1937 克什米尔黄斑蜂

Synonym: Anthidium (Anthidium) nigroventrale

Wu, 1982 黑腹黄斑蜂 [New Synonym]

Distribution: CHINA (Tibet); India.

Niu et al. (2020) Zootaxa 4867(1): 001-067.

\section{Psenidae 三室泥蜂科}

Psenulus ephippius Taylor, Barthélémy, Chi \& Guénard, 2020 马鞍山三室泥蜂 [New Species]

Holotype + (CAS).

Distribution: CHINA (Hong Kong).

Taylor et al. (2020) Journal of Hymenoptera Research 79: 169-211.

Psenulus gibbus Taylor, Barthélémy, Chi \& Guénard, 2020 驼背三室泥蜂 [New Species]

Holotype $q$ (CAS).

Distribution: CHINA (Hong Kong).

Taylor et al. (2020) Journal of Hymenoptera

Research 79: 169-211.

Psenulus pallens Taylor, Barthélémy, Chi \& Guénard, 2020 淡色三室泥蜂 [New Species]

Holotype + (CAS).

Distribution: CHINA (Hong Kong).

Taylor et al. (2020) Journal of Hymenoptera

Research 79: 169-211.

\section{Sphecidae 泥蜂科}

Palmodes mandarinius (F. Smith, 1856)

Synonym: Sphex montanus F. Morawitz, 1889 山

泥蜂 [New Synonym]

Distribution: CHINA (Inner Mongalia, Shanghai, Shanxi); Russia, Tajikistan, Kazakhstan, Mongolia.

Danilov \& Byvaltsev (2020) Zootaxa 4803(3): 401-434.

Palmodes melanarius (Mocsáry, 1883) 黑掌泥蜂 Synonym: Sphex pusillus Gussakovskij, 1930 小 泥蜂 [New Synonym]

Distribution: CHINA (Xinjiang); widely distributed around the world.

Danilov \& Byvaltsev (2020) Zootaxa 4803(3): 401-434.

Palmodes occitanicus (Lepeletier de Saint Fargeau et Audinet-Serville, 1828) 耙掌泥蜂 Synonym: Sphex palmetorum Roth, 1963 棕㭣林 泥蜂 [New Synonym]

Distribution: CHINA (Inner Mongolia, Xinjiang, Shanghai, Zhejiang); widely distributed.

Danilov \& Byvaltsev (2020) Zootaxa 4803(3): 401-434.

\section{Chrysidoidea 青蜂总科}

\section{Bethylidae 肿腿蜂科}

Cephalonomia formosiensis Terayama \& Ho, 2020 台湾头甲肿腿蜂 [New Species]

Holotype $q$ (TARI).

Host: probably the larvae of Sauter anobiids, Falsogastrallus sauteri Pic 寄主可能为档案窃 冨的幼虫.

Distribution: CHINA (Taiwan). 
Ho et al. (2020) Insects 11(4): 223.

Foenobethylus syndesis Chen \& Azevedo, 2020

结合刺股肿腿蜂 [New Species]

Holotype $\hat{\jmath}$ (SYSBM).

Host: Unknown.

Distribution: CHINA (Yunnan).

Chen \& Azevedo (2020) Journal of Asia-Pacific

Entomology 23(4): 1241-1247.

\section{Dryinidae 螯蜂科}

Gonatopus viet Olmi, 1986 越南双距螯蜂 [New

Record]

Host: Stirellus capitatus (Distant, 1918).

Distribution: CHINA (Dong Island of South

China Sea); Vietnam.

Chen et al. (2020) ZooKeys 944: 115-120.

\section{Vespoidea 胡蜂总科}

\section{Formicidae 蚁科}

Lasius chinensis Seifert, 2020 中华毛蚁 [New Species]

Holotype worker ant (SMNG).

Distribution: CHINA (Northeast of China, from east of Qinghai to Shandong).

Seifert (2020) Soil Organisms 92(1): 15-86.

Lasius kabaki Seifert, 2020 卡氏毛蚁 [New Species]

Holotype worker ant (SMNG).

Distribution: CHINA (Sichuan).

Seifert (2020) Soil Organisms 92(1): 15-86.

Lasius longipalpus Seifert, 2020 长角毛蚁 [New

Species]

Holotype worker ant (SMNG).
Distribution: CHINA (Gansu, Qinghai, Shaanxi, Sichuan); Far East, Japan.

Seifert (2020) Soil Organisms 92(1): 15-86.

Lasius sichuense Seifert, 2020 四川毛蚁 [New Species]

Holotype worker ant (SMNG).

Distribution: CHINA (Shaanxi, Sichuan, Yunnan).

Seifert (2020) Soil Organisms 92(1): 15-86.

Myrmecina asiatica Okido, Ogata \& Hosoishi, 2020 亚洲切叶蚁 [New Species]

Holotype worker ant (KUEC).

Distribution: CHINA (Yunnan); Thailand, Malaysia, Indonesia.

Okido, Ogata \& Hosoishi (2020) Bulletin of the Kyushu University Museum (17): 1-108.

Myrmecina asthena Okido, Ogata \& Hosoishi, 2020 弱切叶蚁 [New Species]

Holotype worker ant (KUEC).

Distribution: CHINA (Yunnan); Vietnam, Malaysia.

Okido, Ogata \& Hosoishi (2020) Bulletin of the Kyushu University Museum (17): 1-108.

Ooceraea octoantenna Zhou \& Chen, 2020 八节 卵角蚁 [New Species]

Holotype worker ant (GXNU).

Distribution: CHINA (Guangxi).

Zhou, Chen \& Chen (2020) Sociobiology 67(2): 139-143.

Polyrhachis confusa Wong \& Guénard, 2020 困 惑多刺蚁 [New Species]

Holotype worker ant (IBBL). 
Distribution: CHINA (Hong Kong, Macau).

Wong \& Guénard (2020) Asian Myrmecology 13: e013001.

Polyrhachis fellowesi Wong \& Guénard, 2020 费 氏多刺蚁 [New Species]

Holotype worker ant (IBBL).

Distribution: CHINA (Hong Kong, Guangdong, Guangxi, Zhejiang).

Wong \& Guénard (2020) Asian Myrmecology 13: e013001.

Polyrhachis hunggeuk Wong \& Guénard, 2020 红 腿多刺蚁 [New Species]

Holotype worker ant (IBBL).

Distribution: CHINA (Hong Kong).

Wong \& Guénard (2020) Asian Myrmecology 13: e013001.

Polyrhachis peetersi Wong \& Guénard, 2020 皮 氏多刺蚁 [New Species]

Holotype worker ant (BSM).

Distribution: CHINA (Hong Kong).

Wong \& Guénard (2020) Asian Myrmecology 13: e013001.

Strumigenys subterranea Brassard, Leong \& Guénard, 2020 地层瘤颚蚁 [New Species]

Holotype worker ant (IBBL).

Distribution: CHINA (MaCao.

Brassard et al. (2020) ZooKeys 970: 63-116.

\section{Mutillidae 蚁蜂科}

Ephucilla hejunhuai Lelej, 2020 何俊华蚁蜂

[New Species]

Holotype 9 (IBSS).

Distribution: CHINA (Guangdong).
Lelej (2020) Far Eastern Entomologist 421: 1-9.

Taiwanomyrme cheni Lelej, 2020 陈氏台湾蚁蜂

[New Species]

Holotype $q$ (OLML).

Distribution: CHINA (Yunnan).

Lelej (2020) Far Eastern Entomologist 410: 110.

Mickelomyrme athalia (Pagden, 1934) [New Record]

Distribution: CHINA (Guangdong, Fujian, Yunnan); Malaysia, Thailand, Laos, Vietnam.

Okayasu (2020) Zootaxa 4723(1): 001-110.

Andreimyrme substriolata (Chen, 1957)

Synonym: Smicromyrme tridentiens Chen, 1957

[New Synonym]

Distribution: CHINA (Fujian, Taiwan); Japan, Vietnam, Laos, Thailand, Malaysia, Indonesia.

Okayasu (2020) Zootaxa 4723(1): 001-110.

Nemka wotani (Zavattari, 1913)

Synonym: Smicromyrme (Horaia) chihpenchia Tsuneki, 1993 [New Synonym]

Distribution: CHINA (Taiwan).

Okayasu (2020) Zootaxa 4723(1): 001-110.

Nemka limi (Chen, 1957)

Synonym: Smicromyrme limi nanhai Chen, 1957

[New Synonym]

Distribution: CHINA (Anhui, Shanghai, Zhejiang, Jiangxi, Fujian, Guangdong); Vietnam.

Okayasu (2020) Zootaxa 4723(1): 001-110.

Neotrogaspidia pustulata (Smith, 1873)

Synonym: Smicromyrme yakushimensis Yasumatsu, 1934 [New Synonym] 
曹焕喜, 牛泽清, 唐㒒, 李涛, 朱朝东 (2021) 中国膜翅目 2020 年新分类单元. 生物多样性, 29, 1026-1034.

https://www.biodiversity-science.net/CN/10.17520/biods.2021167

Distribution: CHINA (Taiwan).

Okayasu (2020) Zootaxa 4723(1): 001-110.

\section{Vespidae 胡蜂科}

Allodynerus reduncus Zhang, Chen \& Li, 2020 戈羽

全盾蜾贏 [New Species]

Holotype $q$ (CQNU).

Distribution: CHINA (Yunnan).

Zhang et al. (2020) Zootaxa 4750(4): 545-559.

Allodynerus bimaculus Zhang, Chen \& Li, 2020

双斑全盾蜾贏 [New Species]

Holotype $q$ (CQNU).

Distribution: CHINA (Tibet).

Zhang et al. (2020) Zootaxa 4750(4): 545-559.

Allodynerus asperipunctarus Zhang, Chen \& Li, 2020 糙全盾蜾蠃 [New Species]

Holotype $\widehat{o}$ (CQNU).

Distribution: CHINA (Yunnan).

Zhang et al. (2020) Zootaxa 4750 (4): 545-559.

Ectopioglossa convexa Wang, Chen \& Li, 2020

凸外舌蜾贏 [New Species]

Holotype $\widehat{\delta}$ (CQNU).

Distribution: CHINA (Guangxi).

Wang, Chen \& Li (2020) Journal of Asia-Pacific

Entomology 23(2): 253-259.

Epsilon similimanasicum Zhang, Chen \& Li, 2020 似小式埃蜾蝱 [New Species]

Holotype $\odot$ (CQNU).

Distribution: CHINA (Zhejiang).

Zhang, Chen \& Li (2020) ZooKeys 910: 131-142.
Holotype $q$ (CQNU).

Distribution: CHINA (Guangxi, Yunnan).

Wang, Chen \& Li (2020) Journal of Asia-Pacific

Entomology 23(2): 253-259.

Allorhynchium quadrimaculatum Gusenleitner, 1997 斑异喙蜾贏 [New Record]

Distribution: CHINA (Guangxi, Sichuan, Guizhou, Yunnan); Vietnam, Laos.

Luo et al. (2020) Journal of Hymenoptera

Research 77: 119-137.

Allorhynchium menglianensis (Dong \& Wang, 2017) 预连异嗀蜾蠃 [New Combination]

Halysituberosus menglianensis Dong \& Wang, 2017

Distribution: CHINA (Yunnan).

Luo et al. (2020) Journal of Hymenoptera

Research 77: 119-137.

Allorhynchium van der Vecht, 1963 异喙蜾贏属 Synonym: Halysituberosus Dong \& Wang, 2017

[New Synonym]

Distribition: Oriental, Australian and Palearctic regions.

Luo et al. (2020) Journal of Hymenoptera

Research 77: 119-137.

Allorhynchium lugubrinum (Cameron, 1900)

Synonym: Halysituberosus yingjiangensis Dong \& Wang, 2017 暗异嚎蜾贏 [New Synonym]

Distribution: CHINA (Yunnan); India.

Luo et al. (2020) Journal of Hymenoptera

Research 77: 119-137.

Ectopioglossa trimacula Wang, Chen \& Li, 2020

三斑外舌蜾贏 [New Species] 


\section{Parasitica 寄生部}

\section{Chalcidoidea 小蜂总科}

\section{Aphilinidae 蚜小蜂科}

Coccophagus yunnana Wang, Huang \& Polaszek, 2020 云南食蚧蚜小蜂 [New Species]

Holotype $q$ (FAFU).

Host: an unidentified coccid on Kopsia fruticose

(Coccidae) 红花荵木上的某种蚧虫.

Distribution: CHINA (Yunnan).

Wang et al. (2020) Journal of Natural History 54(29-30): 1879-1896.

Aphytis sankarani Rosen et DeBach, 1986 桑氏 黄蚜小蜂 [New Record]

Host: Pseudaulacaspis cockerelli (Cooley) 考氏 白盾蚧.

Distribution: CHINA (Fujian); India.

$\mathrm{Si}$ et al. (2020) Journal of Environmental Entomology 42(5): 1216-1222.

Coccophagus fumadus

(Hayat, 2010) ?食蚧蚜小蜂 [New Record]

Host: Unknown.

Distribution: CHINA (Hainan); India.

Wang et al. (2020) Journal of Natural History 54(29-30): 1879-1896.

\section{Encyrtidae 跳小蜂科}

Cerapteroceroides guangxiensis $\mathrm{Zu} \& \mathrm{Li}, 2020$ 广 西纹翅跳小蜂 [New Species]

Holotype $q$ (TJAU).

Host: Unknown.

Distribution: CHINA (Tianjin).

$\mathrm{Zu}$ et al. (2020) Journal of Northeast Forestry

University 48(1): 118-119.
Cheiloneurus nankingensis Li \& Xu, 2020 南京 刷盾跳小蜂 [New Species]

Holotype + (NAU).

Host: Aenasius arizonensis Girault attacking Phenacoccus solenopsis Tinsley.

Distribution: CHINA (Jiangsu).

Li et al. (2020) ZooKeys 974: 23-29.

Exoristobia sinica $\mathrm{Zu} \& \mathrm{Li}, 2020$ 中华蝇克跳小 蜂 [New Species]

Holotype $q$ (on slide) (TJAU).

Host: Unknown.

Distribution: CHINA (Tianjin).

$\mathrm{Zu}$ et al. (2020) Journal of Forestry Research.

Metaphycus roseus $\mathrm{Zu}, 2020$ 玫瑰阔柄跳小蜂 [New Species]

Holotype $q$ (on slide) (TJAU).

Host: Antecerococcus roseus (Green) (Hemiptera: Cerococcidae) on Boehmeria nivea (L.) Gaudich. (Urticales: Urticaceae).

Distribution: CHINA (Guangxi).

Zu et al. (2020) Phytoparasitica 48: 43-50.

Microterys perlucidus $\mathrm{Zu}, 2020$ 透翅花翅跳小蜂 [New Species]

Holotype 9 (on slide) (TJAU).

Host: Antecerococcus roseus (Green) (Hemiptera: Cerococcidae) on Boehmeria nivea (L.) Gaudich. (Urticales: Urticaceae).

Distribution: CHINA (Guangxi).

$\mathrm{Zu}$ et al. (2020) Phytoparasitica 48: 43-50.

Rhopus hanni $\mathrm{Zu} \& \mathrm{Li}, 2020$ 韩氏裂脖跳小蜂 [New Species]

Holotype $q$ (NEFU).

Host: Unknown. 
Distribution: CHINA (Tibet).

$\mathrm{Zu}$, Yang \& Li (2020) Journal of Northeast

Forestry University 48(1): 115-117.

Rhopus sulphureus (Westwood, 1837) 硫磺裂脖

跳小蜂 [New Record]

Host: Unknown.

Distribution: CHINA (Heilongjiang); Bulgaria,

Danmark, Germany, Hungary, UK.

$\mathrm{Zu}$, Yang \& Li (2020) Journal of Northeast

Forestry University 48(1): 115-117.

\section{Eulophidae 姬小蜂科}

Oomyzus flavotibialis $\mathrm{Li} \& \mathrm{Li}, 2020$ 黄胫粗脉姬

小蜂 [New Species]

Holotype $q$ (on slide) (NEFU).

Host: Unknown.

Distribution: CHINA (Liaoning, Shandong).

Li \& Li (2020) ZooKeys 950: 41-49.

Oomyzus spiraculus Song, Fei \& Cao, 2020 气门

脊粗脉姬小蜂 [New Species]

Holotype $q$ (IZCAS).

Host: Coccinella septempunctata $\mathrm{L}$. 七星慓虫.

Distribution: CHINA (Jiangsu).

Song et al. (2020) ZooKeys 953: 49-60.

Quadrastichus johnlasallei Gates, Chao, Lin \& Yang, 2020 方强恩胯姬小蜂 [New Species]

Holotype 9 (NCHUC).

Host: Litchiomyia chinensis Yang \& Luo (Diptera: Cecidomyiidae) on Litchi chinensis (Sapindaceae)

荔枝上的中国荔枝瘞蚊.

Distribution: CHINA (Taiwan).

Gates et al. (2020) Journal of Natural History 54(9-12): 635-646.
Quadrastichus lasallei Gates, Chao, Lin \& Yang, 2020 拉萨尔胯姬小蜂 [New Species]

Holotype 9 (NCHUC).

Host: Litchiomyia chinensis Yang \& Luo (Diptera:

Cecidomyiidae) on Litchi chinensis (Sapindaceae)

荔枝上的中国荔枝瘦蚊.

Distribution: CHINA (Taiwan).

Gates et al. (2020) Journal of Natural History 54(9-12): 635-646.

Oomyzus gallerucae (Fonscolombe, 1832) 叶甲 卵粗脉姬小蜂 [New Record]

Host: Unknown from China.

Distribution: CHINA (Liaoning); widely distributed around the world.

Li \& Li (2020) ZooKeys 950: 41-49.

\section{Eupelmidae 旋小蜂科}

Anastatus (Anastatus) flavaeratus Peng \& Tang, 2020 丽头平腹小蜂 [New Species]

Holotype $q$ (FAFU)

Host: Unknown.

Distribution: CHINA (Guangdong).

Peng et al. (2020) Zootaxa 4767(3): 351-401.

Anastatus (Anastatus) pariliquadrus Peng \& Tang, 2020 方头平腹小蜂 [New Species]

Holotype $q$ (FAFU).

Host: Unknown.

Distribution: CHINA (Guangdong).

Peng et al. (2020) Zootaxa 4767(3): 351-401.

\section{Megastigmidae 大疬小蜂科}

Megastigmus zvimendeli Doğanlar \& Hassan 2013

Synonym: Megastigmus sichuanensis Doğanlar \& Zheng 2017 四川大痣小蜂 [New Synonym] 
Distribution: CHINA (Sichuan); Australia, Israel, Turkey.

Le et al. (2020) Bulletin of Entomological Research 110(6): 709-724.

\section{Mymaridae 纱小蜂科}

Lymaenon funiculus Aishan \& Hu, 2020 白索柄 翅缨小蜂 [New Species]

Holotype $q$ (on slide) (ICXU).

Host: Unknown.

Distribution: CHINA (Yunnan, Taiwan).

Aishan et al. (2020) Zootaxa 4834(4): 573-595.

Lymaenon ledongus Aishan \& Hu, 2020 乐东柄

翅缨小蜂 [New Species]

Holotype $q$ (on slide) (ICXU).

Host: Unknown.

Distribution: CHINA (Hainan).

Aishan et al. (2020) Zootaxa 4834(4): 573-595.

Lymaenon longitus Aishan \& Hu, 2020 长尾柄翅 缨小蜂 [New Species]

Holotype $q$ (on slide) (ICXU).

Host: Unknown.

Distribution: CHINA (Hainan).

Aishan et al. (2020) Zootaxa 4834(4): 573-595.

Lymaenon naiquanlini Aishan \& Hu, 2020 林氏 柄翅缨小蜂 [New Species]

Holotype $q$ (on slide) (ICXU).

Host: Unknown.

Distribution: CHINA (Fujian, Taiwan, Tibet, Yunnan).

Aishan et al. (2020) Zootaxa 4834(4): 573-595.

Lymaenon radiculus Aishan \& Hu, 2020 长角柄 翅缨小蜂 [New Species]
Holotype $q$ (on slide) (ICXU).

Host: Unknown.

Distribution: CHINA (Yunnan).

Aishan et al. (2020) Zootaxa 4834(4): 573-595.

Lymaenon zhui Aishan \& Hu, 2020 朱氏柄翅缨

小蜂 [New Species]

Holotype $q$ (on slide) (ICXU).

Host: Unknown.

Distribution: CHINA (Yunnan).

Aishan et al. (2020) Zootaxa 4834(4): 573-595.

Ooctonus exsertus Jin \& Li, 2020 突卵鞘杀卵缨

小蜂 [New Species]

Holotype $q$ (on slide) (NEFU).

Host: Unknown.

Distribution: CHINA (Tibet).

Jin \& Li (2020) Oriental Insects 54(3): 319-326.

\section{Pteromalidae 金小蜂科}

Neocyrtoptyx Xiao \& Yan, 2020 拟赛阿金小蜂 属 [New Genus]

Type species: Neocyrtoptyx shanghensis Xiao \& Yan, 2020; by original designation.

Xiao \& Yan (2020) Zoological Systematics 45(3): 231-235.

Neocyrtoptyx shanghensis Xiao \& Yan, 2020 商 河拟赛阿金小蜂 [New Species]

Host: the larvae of Stenocephus fraxini Wei 白蜡 外齿茎蜂幼虫.

Distribution: CHINA (Shandong).

Xiao \& Yan (2020) Zoological Systematics 45(3): 231-235. Holotype $q$ (IZCAS).

Neocyrtoptyx exilis Xiao \& Yan, 2020 纤拟赛阿 金小蜂 [New Species] 
曹焕喜, 牛泽清, 唐㒒, 李涛, 朱朝东 (2021) 中国膜翅目 2020 年新分类单元. 生物多样性, 29, 1026-1034.

https://www.biodiversity-science.net/CN/10.17520/biods.2021167

Host: the larvae of Stenocephus fraxini Wei 白蜡

外齿茎蜂幼虫.

Distribution: CHINA (Shandong).

Xiao \& Yan (2020) Zoological Systematics 45(3):

231-235. Holotype 9 (IZCAS).

Pteromalus sanjiangyuanicus Yang, 2020 三江源

草原毛虫金小蜂 [New Species]

Holotype $q$ (CAF).

Host: Gynaephora qinghaiensis (Zhou) 青海草原 毛虫.

Distribution: CHINA (Qinghai).

Yang et al. (2020) Scientia Silvae Sinicae 56(2): 99-105.

\section{Trichogrammatidae 赤眼蜂科}

Paracentrobia (Brachistella) bicolor (Girault, 1912) 双色邻赤眼蜂

Synonyms: Japania andoi Ishii, 1938 [New Synonym]; Paracentrobia (Brachistella) garuda Subba Rao, 1974 [New Synonym].

Distribution: CHINA (Taiwan); Japan.

Triapitsyn, Shih \& Huang (2020) Journal of Asia-Pacific Entomology 23(4): 970-980.

Pseudoligosita nephotetticum (Mani, 1939)

Synonym: Oligosita tachikawai Yashiro, 1979

[New Synonym]

Distribution: CHINA (Taiwan); Japan.

Triapitsyn, Shih \& Huang (2020) Journal of Asia-Pacific Entomology 23(4): 970-980.

\section{Cynipoidea 瘦蜂总科}

\section{Cynipidae 瘱蜂科}

Andricus forni Pujade-Villar \& Nicholls, 2020 福 氏栋瘞蜂 [New Species]
Holotype $\odot$ (UB).

Host plant: Quercus glandulifera Blume 包橡.

Distribution: CHINA (Zhejiang).

Pujade-Villar et al. (2020) Zootaxa 4890(4): 554566.

Cerroneuroterus yukawamasudai Pujade-Villar \& Melika, 2020 汤川增田似凹瘞蜂 [New Species]

Holotype $\bigcirc$ (UB).

Host plant: Quercus acutissima Carruthers 麻栋, Quercus variabilis Blume 栓皮栋.

Distribution: CHINA (Shandong).

Pujade-Villar et al. (2020) Zootaxa 4869(4): 515528.

Cycloneuroterus megaformosanus Tang \& Melika, 2020 台湾大青冈瘞蜂 [New Species]

Holotype + (TNMNS).

Host plant: Lithocarpus corneus (Lour.) Rehd. 烟 斗柯.

Distribution: CHINA (Taiwan).

Tang et al. (2020) Proceedings of the Entomological Society of Washington 122(1): 184-196.

Diplolepis abei Pujade-Villar \& Wang, 2020 安 氏玫瑰瘞蜂 [New Species]

Holotype $\bigcirc$ (UB).

Host plant: Chinese Kushui rose, a hybrid of Rosa sertata Rolfe $\times R$. rugosa Thunb. 中国苦水玫瑰. Distribution: CHINA (Gansu).

Pujade-Villar et al. (2020) ZooKeys 904: 131-146.

Heocynips Fang, Nieves-Aldrey \& Melika, 2020 东亚瘞蜂属 [New Genus] 
Type species: Heocynips furvoaurantius Tang,

Nicholls \& Stone, 2020; by original designation and monotypy.

Fang et al. (2020) Proceedings of the

Entomological Society of Washington 122(4): 787-804.

Heocynips furvoaurantius Tang, Nicholls \& Stone, 2020 黑头黄腹东亚㾉蜂 [New Species]

Holotype $q$ (EBRRS).

Host plant: Quercus fabri Hance 白栎; Quercus serrata Thunb. 枹栋.

Distribution: CHINA (Sichuan).

Fang et al. (2020) Proceedings of the

Entomological Society of Washington 122(4): 787-804.

Lithosaphonecrus arcoverticus Liu, Zhu \& Pang, 2020 拱顶柯客瘦蜂 [New Species]

Holotype + (CSCS).

Host plant: Lithocarpus glaber (Thunb.) Nakai 石 栋.

Distribution: CHINA (Hunan).

Yang et al. (2020) Insect Systematics \& Evolution 51: 551-583.

Lithosaphonecrus decarinatus Liu, Zhu \& Pang, 2020 光额柯客瘘蜂 [New Species]

Holotype $q$ (CSCS).

Host plant: Lithocarpus glaber (Thunb.) Nakai 石 栋.

Distribution: CHINA (Hunan).

Yang et al. (2020) Insect Systematics \& Evolution 51: 551-583.

Lithosaphonecrus puigdemonti Pujade-Villar, 2020 蒲氏柯客瘘蜂 [New Species]
Holotype + (UB).

Host plant: Unknown.

Distribution: CHINA (Fujian).

Pujade-Villar, Wang \& Lobato-Vila (2020)

Entomotaxonomia 42(1): 70-80.

Lithosaphonecrus edurus Fang, Melika \& Tang, 2020 粗㾉柯客瘃蜂 [New Species]

Holotype $q$ (EBRRS).

Host plant: Lithocarpus cleistocarpus (Seem.) 峨 眉包果柯.

Distribution: CHINA (Sichuan).

Fang et al. (2020) Proceedings of the

Entomological Society of Washington 122(4): 805-820.

Periclistus orientalis Pang, Liu \& Zhu, 2020 东 方似脊瘞蜂 [New Species]

Holotype $q$ (CSCS).

Distribution: CHINA (Hunan).

Pang et al. (2020) ZooKeys 964: 109-126.

Saphonecrus emarginatus Liu, Zhu \& Pang, 2020 刻腹缺缘客瘘蜂 [New Species]

Holotype $q$ (CSCS).

Host plant: Lithocarpus glaber (Thunb.) Nakai 石 栋.

Distribution: CHINA (Hunan).

Yang et al. (2020) Insect Systematics \& Evolution 51: 551-583.

\section{Figitidae 环腹瘦蜂科}

Leptopilina lasallei Buffington \& Guerrieri, 2020 拉萨尔环腹瘞蜂 [New Species] Holotype $q$ (USNM). 
Host: Drosophila suzukii (Matsumura) and D. pulchrella Sturtevant on Myrica rubra (Lour.) S. et Zucc. 杨梅上的铃木氏果蝇和艳丽果蝇.

Distribution: CHINA (Yunnan).

Buffington et al. (2020) Journal of Natural History 54(9-12): 565-583.

\section{Ichneumonoidea 姬蜂总科}

\section{Braconidae 茧蜂科}

Apanteles albummacula Liu \& Chen, 2020 白斑

线茧蜂 [New Species]

Holotype $q$ (ZJU).

Host: Unknown.

Distribution: CHINA (Zhejiang).

Liu, He \& Chen (2020) Zootaxa 4807(1): 1-205.

Apanteles altus Liu \& Chen, 2020 大刻线茧蜂

[New Species]

Holotype $q$ (ZJU).

Host: Unknown.

Distribution: CHINA (Zhejiang, Fujian, Guangxi, Sichuan).

Liu, He \& Chen (2020) Zootaxa 4807(1): 1-205.

Apanteles ambultor Liu \& Chen, 2020 突变线茧

蜂 [New Species]

Holotype $q$ (ZJU).

Host: Unknown.

Distribution: CHINA (Zhejiang, Taiwan).

Liu, He \& Chen (2020) Zootaxa 4807(1): 1-205.

Apanteles amotus Liu \& Chen, 2020 强凹线茧蜂

[New Species]

Holotype $q$ (ZJU).

Host: Unknown.

Distribution: CHINA (Guangxi).

Liu, He \& Chen (2020) Zootaxa 4807(1): 1-205.
Apanteles angustus Liu \& Chen, 2020 窄区线茧

蜂 [New Species]

Holotype 9 (ZJU).

Host: Unknown.

Distribution: CHINA (Zhejiang, Fujian, Hunan, Jiangsu).

Liu, He \& Chen (2020) Zootaxa 4807(1): 1-205.

Apanteles annosus Liu \& Chen, 2020 皱脸线茧 蜂 [New Species]

Holotype $q$ (ZJU).

Host: Unknown.

Distribution: CHINA (Guangdong).

Liu, He \& Chen (2020) Zootaxa 4807(1): 1-205.

Apanteles aphanofossa Liu \& Chen, 2020 隐沟线 茧蜂 [New Species]

Holotype $q$ (ZJU).

Host: Unknown.

Distribution: CHINA (Zhejiang).

Liu, He \& Chen (2020) Zootaxa 4807(1): 1-205.

Apanteles aspersus Liu \& Chen, 2020 稀刺线茧 蜂 [New Species]

Holotype $q$ (ZJU).

Host: Unknown.

Distribution: CHINA (Zhejiang, Guizhou, Jilin). Liu, He \& Chen (2020) Zootaxa 4807(1): 1-205.

Apanteles attenuator Liu \& Chen, 2020 缢缩线 茧蜂 [New Species]

Holotype $q$ (ZJU).

Host: Unknown.

Distribution: CHINA (Liaoning).

Liu, He \& Chen (2020) Zootaxa 4807(1): 1-205. 
Apanteles bialtus Liu \& Chen, 2020 双高线茧蜂

[New Species]

Holotype $q$ (ZJU).

Host: Unknown.

Distribution: CHINA (Taiwan).

Liu, He \& Chen (2020) Zootaxa 4807(1): 1-205.

Apanteles brevitempus Liu \& Chen, 2020 短规线

茧蜂 [New Species]

Holotype $q$ (ZJU).

Host: Unknown.

Distribution: CHINA (Hunan, Fujian, Guangxi, Taiwan).

Liu, He \& Chen (2020) Zootaxa 4807(1): 1-205.

Apanteles bulbus Liu \& Chen, 2020 泡眼绒茧蜂

[New Species]

Holotype $q$ (ZJU).

Host: Unknown.

Distribution: CHINA (Zhejiang).

Liu, He \& Chen (2020) Zootaxa 4807(1): 1-205.

Apanteles cannabis Liu \& Chen, 2020 麻绳线茧

蜂 [New Species]

Holotype $q$ (ZJU).

Host: Unknown.

Distribution: CHINA (Zhejiang).

Liu, He \& Chen (2020) Zootaxa 4807(1): 1-205.

Apanteles carssus Liu \& Chen, 2020 粗腿线茧蜂

[New Species]

Holotype $q$ (ZJU).

Host: Unknown.

Distribution: CHINA (Anhui, Guangdong, Zhejiang).

Liu, He \& Chen (2020) Zootaxa 4807(1): 1-205.
Apanteles crassus Liu \& Chen, 2020 厚胸线茧蜂

[New Species]

Holotype $q$ (ZJU).

Host: Unknown.

Distribution: CHINA (Fujian, Guangdong, Guizhou).

Liu, He \& Chen (2020) Zootaxa 4807(1): 1-205.

Apanteles distributa Liu \& Chen, 2020 瘤蛾线茧 蜂 [New Species]

Holotype $q$ (ZJU).

Host: Unknown.

Distribution: CHINA (Shandong).

Liu, He \& Chen (2020) Zootaxa 4807(1): 1-205.

Apanteles expansus Liu \& Chen, 2020 后扩线茧 蜂 [New Species]

Holotype $q$ (ZJU).

Host: Unknown.

Distribution: CHINA (Fujian, Guangdong, Hainan, Liaoning, Yunnan, Zhejiang).

Liu, He \& Chen (2020) Zootaxa 4807(1): 1-205.

Apanteles flavibasalis Liu \& Chen, 2020 黄基线 茧蜂 [New Species]

Holotype $q$ (ZJU).

Host: Unknown.

Distribution: CHINA (Guangdong, Guangxi, Guizhou).

Liu, He \& Chen (2020) Zootaxa 4807(1): 1-205.

Apanteles fraxinus Liu \& Chen, 2020 水曲柳线 茧蜂 [New Species]

Holotype $\odot$ (ZJU).

Host: Unknown.

Distribution: CHINA (Heilongjiang).

Liu, He \& Chen (2020) Zootaxa 4807(1): 1-205. 
Apanteles fundum Liu \& Chen, 2020 中脊线茧蜂

[New Species]

Holotype $q$ (ZJU).

Host: Unknown.

Distribution: CHINA (Fujian, Zhejiang).

Liu, He \& Chen (2020) Zootaxa 4807(1): 1-205.

Apanteles fuscidentalis Liu \& Chen, 2020 竹虫线 茧蜂 [New Species]

Holotype $q$ (ZJU).

Host: Unknown.

Distribution: CHINA (Sichuan).

Liu, He \& Chen (2020) Zootaxa 4807(1): 1-205.

Apanteles jasmine Liu \& Chen, 2020 茉莉线茧蜂

[New Species]

Holotype $q$ (ZJU).

Host: Unknown.

Distribution: CHINA (Zhejiang).

Liu, He \& Chen (2020) Zootaxa 4807(1): 1-205.

Apanteles lateroglabris Liu \& Chen, 2020 侧光 线茧蜂 [New Species]

Holotype $q$ (ZJU).

Host: Unknown.

Distribution: CHINA (Heilongjiang, Jilin, Zhejiang).

Liu, He \& Chen (2020) Zootaxa 4807(1): 1-205.

Apanteles limus Liu \& Chen, 2020 横触线茧蜂

[New Species]

Holotype $q$ (ZJU).

Host: Unknown.

Distribution: CHINA (Taiwan).

Liu, He \& Chen (2020) Zootaxa 4807(1): 1-205.
Apanteles longicoxa Liu \& Chen, 2020 长基线茧 蜂 [New Species]

Holotype $q$ (ZJU).

Host: Unknown.

Distribution: CHINA (Fujian, Hainan, Guangxi, Taiwan).

Liu, He \& Chen (2020) Zootaxa 4807(1): 1-205.

Apanteles longidiscus Liu \& Chen, 2020 长胸线 茧蜂 [New Species]

Holotype $q$ (ZJU).

Host: Unknown.

Distribution: CHINA (Liaoning).

Liu, He \& Chen (2020) Zootaxa 4807(1): 1-205.

Apanteles magastigma Liu \& Chen, 2020 大痣线

茧蜂 [New Species]

Holotype $q$ (ZJU).

Host: Unknown.

Distribution: CHINA (Guizhou, Guangxi, Yunnan, Henan, Hainan, Zhejiang, Shandong).

Liu, He \& Chen (2020) Zootaxa 4807(1): 1-205.

Apanteles muscosalis Liu \& Chen, 2020 缀叶蛽 线茧蜂 [New Species]

Holotype $q$ (ZJU).

Host: Unknown.

Distribution: CHINA (Fujian, Zhejiang).

Liu, He \& Chen (2020) Zootaxa 4807(1): 1-205.

Apanteles ocellisublimus Liu \& Chen, 2020 高眼 线茧蜂 [New Species]

Holotype $q$ (ZJU).

Host: Unknown.

Distribution: CHINA (Fujian, Hubei).

Liu, He \& Chen (2020) Zootaxa 4807(1): 1-205. 
Apanteles plureseta Liu \& Chen, 2020 毛点线茧

蜂 [New Species]

Holotype $q$ (ZJU).

Host: Unknown.

Distribution: CHINA (Zhejiang).

Liu, He \& Chen (2020) Zootaxa 4807(1): 1-205.

Apanteles prominens Liu \& Chen, 2020 突项线 独蜂 [New Species]

Holotype $q$ (ZJU).

Host: Unknown.

Distribution: CHINA (Zhejiang, Guangdong, Hunan).

Liu, He \& Chen (2020) Zootaxa 4807(1): 1-205.

Apanteles quadra Liu \& Chen, 2020 方脸线茧蜂

[New Species]

Holotype $\bigcirc$ (ZJU).

Host: Unknown.

Distribution: CHINA (Guizhou, Hainan).

Liu, He \& Chen (2020) Zootaxa 4807(1): 1-205.

Apanteles radocoxa Liu \& Chen, 2020 刮基线茧 蜂 [New Species]

Holotype $q$ (ZJU).

Host: Unknown.

Distribution: CHINA (Guangxi).

Liu, He \& Chen (2020) Zootaxa 4807(1): 1-205.

Apanteles salsala Liu \& Chen, 2020 弄蝶线茧蜂

[New Species]

Holotype $q$ (ZJU).

Host: Unknown.

Distribution: CHINA (Zhejiang, Fujian).

Liu, He \& Chen (2020) Zootaxa 4807(1): 1-205.
Apanteles scrobiculatus Liu \& Chen, 2020 网纹 线茧蜂 [New Species]

Holotype $q$ (ZJU).

Host: Unknown.

Distribution: CHINA (Hainan, Guizhou, Zhejiang, Yunnan).

Liu, He \& Chen (2020) Zootaxa 4807(1): 1-205.

Apanteles semicarinatus Liu \& Chen, 2020 半脊 线茧蜂 [New Species]

Holotype $q$ (ZJU).

Host: Unknown.

Distribution: CHINA (Liaoning, Jilin, Heilongjiang, Shandong, Henan, Hubei, Anhui, Guangdong, Guizhou, Fujian, Zhejiang).

Liu, He \& Chen (2020) Zootaxa 4807(1): 1-205.

Apanteles stigmaium Liu \& Chen, 2020 小痣线 独蜂 [New Species]

Holotype $\uparrow$ (ZJU).

Host: Unknown.

Distribution: CHINA (Jilin, Zhejiang).

Liu, He \& Chen (2020) Zootaxa 4807(1): 1-205.

Apanteles tenuis Liu \& Chen, 2020 条带线茧蜂 [New Species]

Holotype $q$ (ZJU).

Host: Unknown.

Distribution: CHINA (Yunnan, Zhejiang, Hunan, Henan, Taiwan).

Liu, He \& Chen (2020) Zootaxa 4807(1): 1-205.

Apanteles alticella Liu \& Chen, 2020 高室线茧 蜂 [New Species]

Holotype + (ZJU).

Host: Unknown.

Distribution: CHINA (Hainan). 
Liu, He \& Chen (2020) Zootaxa 4807(1): 1-205.

Apanteles aphanoiugum Liu \& Chen, 2020 隐脊 线茧蜂 [New Species]

Holotype $q$ (ZJU).

Host: Unknown.

Distribution: CHINA (Yunnan, Zhejiang, Guizhou).

Liu, He \& Chen (2020) Zootaxa 4807(1): 1-205.

Apanteles cavitergita Liu \& Chen, 2020 凹背线 茧蜂 [New Species]

Holotype $q$ (ZJU).

Host: Unknown.

Distribution: CHINA (Xinjiang).

Liu, He \& Chen (2020) Zootaxa 4807(1): 1-205.

Apanteles impunctus Liu \& Chen, 2020 无刻线茧

蜂 [New Species]

Holotype $q$ (ZJU).

Host: Unknown.

Distribution: CHINA (Gansu).

Liu, He \& Chen (2020) Zootaxa 4807(1): 1-205.

Apanteles lineatella Liu \& Chen, 2020 麦蛾线茧

蜂 [New Species]

Holotype $q$ (ZJU).

Host: Unknown.

Distribution: CHINA (Xinjiang).

Liu, He \& Chen (2020) Zootaxa 4807(1): 1-205.

Apanteles longiala Liu \& Chen, 2020 长翅线茧 蜂 [New Species]

Holotype $q$ (ZJU).

Host: Unknown.

Distribution: CHINA (Zhejiang).

Liu, He \& Chen (2020) Zootaxa 4807(1): 1-205.
Apanteles palliditegula Liu \& Chen, 2020 浅翅基 线茧蜂 [New Species]

Holotype $q$ (ZJU).

Host: Unknown.

Distribution: CHINA (Guangdong).

Liu, He \& Chen (2020) Zootaxa 4807(1): 1-205.

Apanteles rectala Liu \& Chen, 2020 直翅线茧蜂

[New Species]

Holotype $q$ (ZJU).

Host: Unknown.

Distribution: CHINA (Guizhou, Yunnan, Zhejiang, Shaanxi).

Liu, He \& Chen (2020) Zootaxa 4807(1): 1-205.

Apanteles rhombos Liu \& Chen, 2020 菱区线茧 蜂 [New Species]

Holotype $q$ (ZJU).

Host: Unknown.

Distribution: CHINA (Zhejiang, Yunnan).

Liu, He \& Chen (2020) Zootaxa 4807(1): 1-205.

Apanteles setosus Liu \& Chen, 2020 毛背线茧蜂

[New Species]

Holotype 9 (ZJU).

Host: Unknown.

Distribution: CHINA (Guangdong, Fujian, Hainan, Sichuan, Zhejiang).

Liu, He \& Chen (2020) Zootaxa 4807(1): 1-205.

Aspicolpus clypeconcavus Yan \& Chen, 2020 凹 唇盾茧蜂 [New Species]

Holotype 9 (ZJU).

Host: Unknown.

Distribution: CHINA (Zhejiang).

Yan et al. (2020) Zootaxa 4743(3): 391-401. 
Aspicolpus frontirugatus Yan \& Chen, 2020 皱额

盾茧蜂 [New Species]

Holotype $q$ (ZJU).

Host: Unknown.

Distribution: CHINA (Tibet).

Yan et al. (2020) Zootaxa 4743(3): 391-401.

Aspicolpus jilinensis Yan \& Chen, 2020 吉林盾

茧蜂 [New Species]

Holotype $q$ (ZJU).

Host: Unknown.

Distribution: CHINA (Jilin).

Yan et al. (2020) Zootaxa 4743(3): 391-401.

Aspicolpus striatus Yan \& Chen, 2020 条纹盾茧 蜂 [New Species]

Holotype $q$ (ZJU).

Host: Unknown.

Distribution: CHINA (Fujian).

Yan et al. (2020) Zootaxa 4743(3): 391-401.

Atanycolus carinatus Li, He \& Chen, 2020 强脊 刻柄茧蜂 [New Species]

Holotype $q$ (ZJU).

Host: Unknown.

Distribution: CHINA (Yunnan).

Li, He \& Chen (2020) Zootaxa 4818(1): 1-74.

Atanycolus longiquus Li, He \& Chen, 2020 长规

刻柄茧蜂 [New Species]

Holotype $q$ (ZJU).

Host: Unknown.

Distribution: CHINA (Yunnan).

Li, He \& Chen (2020) Zootaxa 4818(1): 1-74.
Atanycolus maculipennis Li, He \& Chen, 2020 斑 翅刻柄茧蜂 [New Species]

Holotype $q$ (ZJU).

Host: Unknown.

Distribution: CHINA (Yunnan).

Li, He \& Chen (2020) Zootaxa 4818(1): 1-74.

Atanycolus setosus Li, He \& Chen, 2020 密毛刻 柄茧蜂 [New Species]

Holotype $q$ (ZJU).

Host: Unknown.

Distribution: CHINA (Yunnan).

Li, He \& Chen (2020) Zootaxa 4818(1): 1-74.

Bracon (Glabrobracon) indistinctus Li, He \& Chen, 2020 浅沟光茧蜂 [New Species]

Holotype $q$ (ZJU).

Host: Unknown.

Distribution: CHINA (Zhejiang).

Li, He \& Chen (2020) Deutsche Entomologische

Zeitschrift 67: 209-252.

Bracon (Glabrobracon) leptotes Li, He \& Chen, 2020 细管光茧蜂 [New Species]

Holotype $q$ (ZJU).

Host: Unknown.

Distribution: CHINA (Fujian).

Li, He \& Chen (2020) Deutsche Entomologische

Zeitschrift 67: 209-252.

Bracon (Glabrobracon) longistriatus Li, He \& Chen, 2020 纵条光茧蜂 [New Species]

Holotype $q$ (ZJU).

Host: Unknown.

Distribution: CHINA (Heilongjiang).

Li, He \& Chen (2020) Deutsche Entomologische

Zeitschrift 67: 209-252. 
Bracon (Glabrobracon) megaventris Li, He \&

Chen, 2020 大腹光茧蜂 [New Species]

Holotype $q$ (ZJU).

Host: Unknown.

Distribution: CHINA (Henan).

Li, He \& Chen (2020) Deutsche Entomologische

Zeitschrift 67: 209-252.

Bracon (Lucobracon) brevicarinatus $\mathrm{Li}, \mathrm{He} \&$ Chen, 2020 短脊宽口茧蜂 [New Species]

Holotype $\bigcirc$ (ZJU).

Host: Unknown.

Distribution: CHINA (Liaoning).

Li, He \& Chen (2020) Deutsche Entomologische

Zeitschrift 67: 209-252.

Bracon (Lucobracon) coarctatus Li, He \& Chen, 2020 扁胸宽口茧蜂 [New Species]

Holotype $q$ (ZJU).

Host: Unknown.

Distribution: CHINA (Jilin).

Li, He \& Chen (2020) Deutsche Entomologische

Zeitschrift 67: 209-252.

Bracon (Lucobracon) curculiovorus Li, He \& Chen, 2020 象甲宽口茧蜂 [New Species]

Holotype $q$ (ZJU).

Host: Unknown.

Distribution: CHINA (Inner Mongolia).

Li, He \& Chen (2020) Deutsche Entomologische

Zeitschrift 67: 209-252.

Bracon (Lucobracon) flavitestaceus $\mathrm{Li}, \mathrm{He} \&$ Chen, 2020 黄褐宽口茧蜂 [New Species]

Holotype $q$ (ZJU).

Host: Unknown.

Distribution: CHINA (Jilin, Liaoning).
Li, He \& Chen (2020) Deutsche Entomologische

Zeitschrift 67: 209-252.

Bracon (Lucobracon) quadratus Li, He \& Chen, 2020 方斑宽口茧蜂 [New Species]

Holotype $\bigcirc$ (ZJU).

Host: Unknown.

Distribution: CHINA (Liaoning).

Li, He \& Chen (2020) Deutsche Entomologische

Zeitschrift 67: 209-252.

Bracon (Uncobracon) eurysulcatus Li, He \& Chen, 2020 宽沟颚钩茧蜂 [New Species]

Holotype $\bigcirc$ (ZJU).

Host: Unknown.

Distribution: CHINA (Guangxi).

Li, He \& Chen (2020) Deutsche Entomologische Zeitschrift 67: 209-252.

Bracon (Uncobracon) longwangshanensis $\mathrm{Li}, \mathrm{He}$ \& Chen, 2020 龙王山颔钩茧蜂 [New Species]

Holotype + (ZJU).

Host: Unknown.

Distribution: CHINA (Zhejiang).

Li, He \& Chen (2020) Deutsche Entomologische

Zeitschrift 67: 209-252.

Campyloneurus brachyurus Li, van Achterberg \& Chen, 2020 短尾弯肘茧蜂 [New Species]

Holotype $ᄋ$ (ZJU).

Host: Unknown.

Distribution: CHINA (Hunan).

Li, van Achterbert \& Chen (2020) Zootaxa 4884(1): 1-67.

Campyloneurus lasiofacialis $\mathrm{Li}$, van Achterberg \& Chen, 2020 毛面弯肘茧蜂 [New Species]

Holotype $\bigcirc$ (ZJU). 
Host: Unknown.

Distribution: CHINA (Hunan).

Li, van Achterbert \& Chen (2020) Zootaxa 4884(1): 1-67.

Campyloneurus longitergum $\mathrm{Li}$, van Achterberg \& Chen, 2020 长背深沟茧蜂 [New Species]

Holotype $\bigcirc$ (ZJU).

Host: Unknown.

Distribution: CHINA (Yunnan).

Li, van Achterbert \& Chen (2020) Zootaxa 4884(1): 1-67.

Campyloneurus longitudinalis Li, van Achterberg

\& Chen, 2020 纵条念珠茧蜂 [New Species]

Holotype $q$ (ZJU).

Host: Unknown.

Distribution: CHINA (Yunnan).

Li, van Achterbert \& Chen (2020) Zootaxa 4884(1): 1-67.

Campyloneurus micromacularis $\mathrm{Li}$, van Achterberg \& Chen, 2020 小斑深沟茧蜂 [New Species]

Holotype $q$ (ZJU).

Host: Unknown.

Distribution: CHINA (Yunnan).

Li, van Achterbert \& Chen (2020) Zootaxa 4884(1): 1-67.

Campyloneurus nigriventris $\mathrm{Li}$, van Achterberg \& Chen, 2020 黑腹弯肘茧蜂 [New Species]

Holotype $\bigcirc$ (ZJU).

Host: Unknown.

Distribution: CHINA (Yunnan).

Li, van Achterbert \& Chen (2020) Zootaxa 4884(1): 1-67.
Campyloneurus pachypus Li, van Achterberg \& Chen, 2020 粗柄弯肘茧蜂 [New Species]

Holotype $ᄋ$ (ZJU).

Host: Unknown.

Distribution: CHINA (Hebei).

Li, van Achterbert \& Chen (2020) Zootaxa 4884(1): 1-67.

Campyloneurus promiscuus Li, van Achterberg \& Chen, 2020 混纹弯肘茧蜂 [New Species]

Holotype $ᄋ$ (ZJU).

Host: Semanotus bifasciatus M. 双条杉天牛.

Distribution: CHINA (Guangdong).

Li, van Achterbert \& Chen (2020) Zootaxa 4884(1): 1-67.

Campyloneurus quadraticeps Li, van Achterberg \& Chen, 2020 方头弯肘茧蜂 [New Species]

Holotype $q$ (ZJU).

Host: Unknown.

Distribution: CHINA (Fujian).

Li, van Achterbert \& Chen (2020) Zootaxa 4884(1): 1-67.

Campyloneurus robusticella $\mathrm{Li}$, van Achterberg \& Chen, 2020 粗室深沟茧蜂 [New Species]

Holotype $\bigcirc$ (ZJU).

Host: Unknown.

Distribution: CHINA (Hunan).

Li, van Achterbert \& Chen (2020) Zootaxa 4884(1): 1-67.

Campyloneurus rugifacialis $\mathrm{Li}$, van Achterberg \& Chen, 2020 皱颜深沟茧蜂 [New Species]

Holotype $\bigcirc$ (ZJU).

Host: Unknown.

Distribution: CHINA (Guangxi). 
Li, van Achterbert \& Chen (2020) Zootaxa 4884(1): 1-67.

Campyloneurus stigmosus $\mathrm{Li}$, van Achterberg \& Chen, 2020 多斑深沟茧蜂 [New Species]

Holotype $q$ (ZJU).

Host: Unknown.

Distribution: CHINA (Guangdong, Fujian).

Li, van Achterbert \& Chen (2020) Zootaxa 4884(1): 1-67.

Campyloneurus tergipunctatus $\mathrm{Li}$, van Achterberg \& Chen, 2020 刻背深沟茧蜂 [New Species]

Holotype $q$ (ZJU).

Host: Unknown.

Distribution: CHINA (Yunnan).

Li, van Achterbert \& Chen (2020) Zootaxa 4884(1): 1-67.

Craspedolcus maculithorax $\mathrm{Li}, \mathrm{He} \&$ Chen, 2020

斑胸缘柄茧蜂 [New Species]

Holotype $q$ (ZJU).

Host: Unknown.

Distribution: CHINA (Fujian).

Li, He \& Chen (2020) Zootaxa 4818(1): 1-74.

Craspedolcus nigrabdominalis $\mathrm{Li}, \mathrm{He} \&$ Chen, 2020 黑腹缘柄茧蜂 [New Species]

Holotype $q$ (ZJU).

Host: Unknown.

Distribution: CHINA (Tibet).

Li, He \& Chen (2020) Zootaxa 4818(1): 1-74.

Deuterixys jilinensis Zheng \& Song, 2020 吉林背 腰茧蜂 [New Species]

Holotype $q$ (FAFU).

Host: Unknown.
Distribution: CHINA (Jilin).

Zheng \& Song (2020) Zootaxa 4728(4): 489-497.

Deuterixys longitarsus Zheng \& Song, 2020 长跗

背腰茧蜂 [New Species]

Holotype $q$ (FAFU).

Host: Unknown.

Distribution: CHINA (Fujian).

Zheng \& Song (2020) Zootaxa 4728(4): 489-497.

Deuterixys shennonggensis Zheng \& Song, 2020

神龙架背腰茧蜂 [New Species]

Holotype $q$ (FAFU).

Host: Unknown.

Distribution: CHINA (Hubei).

Zheng \& Song (2020) Zootaxa 4728(4): 489-497.

Ecphylus lini Belokobylskij, 2020 林氏异腹茧蜂

[New Species]

Holotype 9 (TNMNS).

Host: Scolytus japonicus Chapuis 果树小蛽.

Distribution: CHINA (Taiwan).

Belokobylskij \& Lin (2020) Zoosystematica

Rossica 29(1): 23-32.

Ectemnoplax bimaculosus $\mathrm{Li}, \mathrm{He} \&$ Chen, 2020 双斑埃茧蜂 [New Species]

Holotype $q$ (ZJU).

Host: Unknown.

Distribution: CHINA (Yunnan).

Li, He \& Chen (2020) Zootaxa 4818(1): 1-74.

Glyptapanteles gigas Liang \& Song, 2020 巨网 苔蛾刻线茧蜂 [New Species]

Holotype $q$ (FAFU).

Host: Macrobrochis gigas Walker 巨网苔蛾.

Distribution: CHINA (Fujian). 
Lu et al. (2020) ZooKeys 913: 127-139.

Indabracon albogilvus Li, Tang \& Chen, 2020 黄 白印度茧蜂 [New Species]

Holotype $q$ (ZJU).

Host: Unknown.

Distribution: CHINA (Yunnan).

Li, Tang \& Chen (2020) Journal of Hymenoptera

Research 76: 39-56.

Indabracon discolor $\mathrm{Li}$, Tang \& Chen, 2020 异色 印度茧蜂 [New Species]

Holotype $q$ (ZJU).

Host: Unknown.

Distribution: CHINA (Yunnan).

Li, Tang \& Chen (2020) Journal of Hymenoptera Research 76: 39-56.

Indabracon nigricans $\mathrm{Li}$, Tang \& Chen, 2020 黑 背印度茧蜂 [New Species]

Holotype $q$ (ZJU).

Host: Unknown.

Distribution: CHINA (Yunnan).

Li, Tang \& Chen (2020) Journal of Hymenoptera

Research 76: 39-56.

Indabracon semicircularis $\mathrm{Li}$, Tang \& Chen, 2020 半圆印度茧蜂 [New Species]

Holotype $q$ (ZJU).

Host: Unknown.

Distribution: CHINA (Yunnan).

Li, Tang \& Chen (2020) Journal of Hymenoptera

Research 76: 39-56.

Iphiaulax longinervis $\mathrm{Li}$, van Achterberg \& Chen, 2020 长脉深沟茧蜂 [New Species]

Holotype $q$ (ZJU).
Host: Unknown.

Distribution: CHINA (Yunnan).

Li, van Achterbert \& Chen (2020) Zootaxa 4884(1): 1-67.

Lipolexis peregrinus Tomanović \& Kocić, 2020 疑源长径蚜茧蜂 [New Species]

Holotype $q$ (FBUB).

Host: Myzus persicae (Sulzer) 桃蚜, Aphis sp., A. gossypii Glover 棉蚜, Toxoptera aurantii (Boyer de Fonscolomber) 茶蚜, Melanaphis sacchari (Zehntner) 高粱蚜.

Distribution: CHINA (Beijing).

Kocić et al. (2020) Insects 11(10): 667.

Lipolexis takadai Tomanović \& Kocić, 2020 高 田氏长径蚜茧蜂 [New Species]

Holotype + $(\mathrm{CNC})$.

Host: Aphis gossypii Glover 棉蚜.

Distribution: CHINA (Shaanxi).

Kocić et al. (2020) Insects 11(10): 667.

Myiocephalus cracentis $\mathrm{Li}, 2020$ 修长突眼茧蜂 [New Species]

Holotype $q$ (FAFU).

Host: Unknown.

Distribution: CHINA (Ningxia, Hubei).

Li et al. (2020) ZooKeys 933: 95-105.

Neognamptodon Belokobylskij, 1999 新塬腹茧 蜂属 [New Record Genus]

Distribution: CHINA (Yunnan); Madagascar, Papua New Guinea.

Tian et al. (2020) Zootaxa 4778(3): 471-508.

Neognamptodon laticauda Tan \& van Achterberg, 2020 宽尾新塬腹茧蜂 [New Species] 
Holotype $\odot$ (NWUX).

Host: Unknown.

Distribution: CHINA (Yunnan).

Tian et al. (2020) Zootaxa 4778(3): 471-508.

Parabobekoides van Achterberg \& Tan, 2020 副 波贝茧蜂亚属 [New Subgenus]

Type species: Separatatus (Parabobekoides) yinshani Zhang \& van Achterberg, 2020; by original designation.

Host: Unknown.

Distribution: CHINA (Shaanxi).

Zhang et al. (2020) ZooKeys 926: 25-51.

Separatatus (Parabobekoides) yinshani Zhang \& van Achterberg, 2020 银山裂腹反频茧蜂 [New Species]

Holotype $\odot$ (NWUX).

Host: Unknown.

Distribution: CHINA (Shaanxi).

Zhang et al. (2020) ZooKeys 926: 25-51.

Praon genriki Davidian, 2020 亨氏蚜外茧蜂

[New Species]

Holotype $q(\mathrm{ZIN})$.

Host: Unknown.

Distribution: CHINA (Yunnan).

Davidian (2020) Caucasian Entomological

Bulletin 16(2): 207-211.

Praon longistigmus Davidian, 2020 长痣蚜外茧 蜂 [New Species]

Holotype $\widehat{o}$ (ZIN).

Distribution: CHINA (Sichuan).

Davidian (2020) Caucasian Entomological

Bulletin 16(2): 207-211.
Pseudocyanopterus van Achterberg, Cao \& Yang, 2020 伪中脊茧蜂属 [New Genus]

Type species: Pseudocyanopterus raddeivorus Cao, van Achterberg \& Yang, 2020; by original designation and monotypy.

Distribution: CHINA (Liaoning).

Cao et al. (2020) Zootaxa 4881(1): 104-130.

Pseudocyanopterus raddeivorus Cao, van Achterberg \& Yang, 2020 栗山天牛伪中脊茧蜂 [New Species]

Holotype $q$ (CAF).

Host: Massicus raddei (Blessig \& Solsky, 1872) 栗山天牛.

Distribution: CHINA (Liaoning).

Cao et al. (2020) Zootaxa 4881(1): 104-130.

Pseudognaptodon Fischer, 1965 拟塬腹茧蜂属 [New Record Genus]

Distribution: the New World; CHINA (Shaanxi) and Vietnam in the Old World.

Tian et al. (2020) Zootaxa 4778(3): 471-508.

Pseudognaptodon sinensis Tan \& van Achterberg, 2020 中华拟塬腹茧蜂 [New Species]

Holotype 9 (NWUX).

Host: Unknown.

Distribution: CHINA (Shaanxi).

Tian et al. (2020) Zootaxa 4778(3): 471-508.

Rilipertus Haeselbarth，1996 宽鞘缘茧蜂属 [New Record Genus]

Distribution: CHINA (Hubei).

Li, Zheng \& Chen (2020) Zootaxa 4851(1): 198200. 
曹焕喜, 牛泽清, 唐㒒, 李涛, 朱朝东 (2021) 中国膜翅目 2020 年新分类单元. 生物多样性, 29, 1026-1034.

https://www.biodiversity-science.net/CN/10.17520/biods.2021167

Rilipertus zhongnanshanius Li, 2020 钟氏宽鞘缘

独蜂 [New Species]

Holotype $q$ (FAFU).

Host: Unknown.

Distribution: CHINA (Hubei).

Li, Zheng \& Chen (2020) Zootaxa 4851(1): 198200.

Shelfordia fulva Li, He \& Chen, 2020 黄褐纹腹

独蜂 [New Species]

Holotype $q$ (ZJU).

Host: Unknown.

Distribution: CHINA (Yunnan).

Li, He \& Chen (2020) Zootaxa 4818(1): 1-74.

Shelfordia obscuripennis Li, He \& Chen, 2020 暗 翅纹腹茧蜂 [New Species]

Holotype $q$ (ZJU).

Host: Unknown.

Distribution: CHINA (Hainan).

Li, He \& Chen (2020) Zootaxa 4818(1): 1-74.

Stenobracon (Stenobracon) longatus Li, He \& Chen, 2020 长室窄茧蜂 [New Species]

Holotype + (ZJU).

Host: Unknown.

Distribution: CHINA (Zhejiang).

Li, He \& Chen (2020) Zootaxa 4818(1): 1-74.

Vipio abnormis Li, He \& Chen, 2020 缺区簇毛 茧蜂 [New Species]

Holotype $q$ (ZJU).

Host: Unknown.

Distribution: CHINA (Inner Mongolia).

Li, He \& Chen (2020) Zootaxa 4818(1): 1-74.
Apanteles argiope Nixon, 1965 金蛛线茧蜂 [New Record]

Distribution: CHINA (Fujian, Guangdong, Guangxi, Hainan, Yunnan, Zhejiang); Australia, Fiji, India, Indonesia, Malaysia, Philippines, Singapore, South Korea, Vanuatu.

Liu, He \& Chen (2020) Zootaxa 4807(1): 1-205.

Apanteles brunnistigma Abdinbekova, 1969 褐痣 线茧蜂 [New Record]

Distribution: CHINA (Fujian, Zhejiang); Azerbaijan, Canary, Czechoslovakia, Finland, Hungary, Islands, Italy, Lithuania, Russia, Switzerland, Turkey, United Kingdom.

Liu, He \& Chen (2020) Zootaxa 4807(1): 1-205.

Apanteles cerberus Nixon, 1965 瑟伯罗斯线茧 蜂 [New Record]

Distribution: CHINA (Beijing, Guangdong, Zhejiang); India.

Liu, He \& Chen (2020) Zootaxa 4807(1): 1-205.

Apanteles chloris Nixon, 1965 克洛丽丝线茧蜂 [New Record]

Distribution: CHINA (Fujian, Guangdong, Guizhou, Zhejiang); Philippines, Vietnam.

Liu, He \& Chen (2020) Zootaxa 4807(1): 1-205.

Apanteles chloris Nixon, 1965 克洛丽丝绒茧蜂 Synonym: Apanteles cornicula Chen \& Song, 2004 [New Synonym]

Distribution: CHINA (Fujian, Guangdong, Guizhou, Zhejiang); Philippines, Vietnam.

Liu, He \& Chen (2020) Zootaxa 4807(1): 1-205.

Apanteles cocotis Wilkinson, 1934 椰树线茧蜂 [New Record] 
曹焕喜, 牛泽清, 唐璞, 李涛, 朱朝东 (2021) 中国膜翅目 2020 年新分类单元. 生物多样性, 29, 1026-1034.

https://www.biodiversity-science.net/CN/10.17520/biods.2021167

Distribution: CHINA (Fujian, Guangdong,

Guangxi, Guizhou, Taiwan, Zhejiang); Indonesia.

Liu, He \& Chen (2020) Zootaxa 4807(1): 1-205.

Apanteles demades Nixon, 1965 德马德斯绒茧

蜂 [New Record]

Distribution: CHINA (Hainan); Malaysia,

Vietnam.

Liu, He \& Chen (2020) Zootaxa 4807(1): 1-205.

Apanteles medon Nixon, 1965 米登线茧蜂 [New

Record]

Distribution: CHINA (Chongqing, Fujian,

Guangdong, Guizhou, Hainan, Henan, Hunan,

Yunnan, Zhejiang); Malaysia, Vietnam.

Liu, He \& Chen (2020) Zootaxa 4807(1): 1-205.

Apanteles opacus (Ashmead, 1905) 棉大卷叶螟 线茧蜂

Synonyms: Apanteles cavatiptera Chen \& Song, 2004 [New Synonym]; Apanteles glacilipes Song

\& Chen, 2004 [New Synonym]

Distribution: CHINA (Anhu, Chongqing, Fujian, Guangdong, Guangxi, Guizhou, Hainan, Henan, Hubei, Hunan, Jiangsu, Liaoning, Shaanxi, Shanghai, Sichuan, Taiwan, Yunnan, Zhejiang); India, Japan, Malaysia, Philippines, Vietnam.

Liu, He \& Chen (2020) Zootaxa 4807(1): 1-205.

Apanteles psenes Nixon, 1965 浅跗线茧蜂 [New

Record]

Distribution: CHINA (Zhejiang); Malaysia, Vietnam.

Liu, He \& Chen (2020) Zootaxa 4807(1): 1-205.

Apanteles saravus Nixon, 1965 萨拉乌斯线茧蜂 [New Record]
Distribution: CHINA (Fujian, Guangdong, Guangxi, Guizhou, Hainan, Hunan, Yunnan, Zhejiang); Philippines, Vietnam.

Liu, He \& Chen (2020) Zootaxa 4807(1): 1-205.

Apanteles sartamus Nixon, 1965 安全线茧蜂 [New Record]

Distribution: CHINA (Guangdong, Guangxi, Taiwan); Philippines.

Liu, He \& Chen (2020) Zootaxa 4807(1): 1-205.

Apanteles usipetes Nixon, 1965 乌斯佩特线茧蜂 [New Record]

Distribution: CHINA (Fujian, Sichuan); Malaysia. Liu, He \& Chen (2020) Zootaxa 4807(1): 1-205.

Campyloneurus kirbyi (Cameron, 1905) 科氏弯 肘茧蜂 [New Record]

Distribution: CHINA (Yunnan); Sri Lanka.

Li, van Achterberg \& Chen (2020) Zootaxa 4884(1): 1-67.

Elasmosoma (Elasmosoma) pergandei Ashmead, 1895 [New Record]

Distribution: CHINA (Heilongjiang); Mongolia, Tajikistan, Canada, U. S. A.

Li et al. (2020) Zoological Systematics 45(4): 281-289.

Neoneurus Haliday, 1838 [New Record Genus] Distribution. Palaearctic and Nearctic Regions. Li et al. (2020) Zoological Systematics 45(4): 281-289.

Neoneurus clypeatus (Foerster, 1863) [New Record]

Distribution: CHINA (Heilongjiang); Mongolia, Kazakhstan, Korea, Europe. 
Li et al. (2020) Zoological Systematics 45(4): 281-289.

Shelfordia longicaudata van Achterberg, 1993 长 尾纹腹茧蜂 [New Record]

Distribution: CHINA (Yunnan).

Li, He \& Chen (2020) Zootaxa 4818(1): 1-74.

Vipio mongolicus Telenga, 1936 蒙古簇毛茧蜂 [New Record]

Distribution: CHINA (Hebei, Heilongjiang, Nei Menggu, Ningxia).

Li, He \& Chen (2020) Zootaxa 4818(1): 1-74.

Elasmosoma (Sinoneoneurus) obscuripennis (He,

Chen \& van Achterberg, 1997) [New Combination]

Sinoneoneurus obscuripennis He, Chen \& van Achterberg, 1997

Distribution: CHINA (East Palaearctic Region).

Li et al. (2020) Zoological Systematics 45(4): 281-289.

Elasmosoma (Sinoneoneurus) pallidipennis (He,

Chen \& van Achterberg, 1997) [New Combination]

Sinoneoneurus pallidipennis He, Chen \& van Achterberg, 1997

Distribution: CHINA (East Palaearctic Region).

Li et al. (2020) Zoological Systematics 45(4): 281-289.

Paroligoneurus cosmopterygivorus (He, 2000) 竹 尖蛾稀脉茧蜂 [New Combination]

Oligoneurus cosmopterygivorus $\mathrm{He}, 2000$ 竹尖蛾

寡脉茧蜂

Distribution: CHINA (Zhejiang).
Ranjith et al. (2020) Zootaxa 4786 (3): 396-408.

Paroligoneurus crassicornis (He, 2000) 粗角稀 脉茧蜂 [New Combination]

Oligoneurus crassicornis He, 2000 粗角寡脉茧 蜂

Distribution: CHINA (Zhejiang).

Ranjith et al. (2020) Zootaxa 4786 (3): 396-408.

Paroligoneurus flavifacialis (He, 2000) 黄脸稀 脉茧蜂 [New Combination]

Oligoneurus flavifacialis He, 2000 黄脸寡脉茧 蜂

Distribution: CHINA (Zhejiang).

Ranjith et al. (2020) Zootaxa 4786 (3): 396-408.

Paroligoneurus sinensis (He, 2000) 中华稀脉茧 蜂 [New Combination]

Oligoneurus sinensis He, 2000 中华寡脉茧蜂

Distribution: CHINA (Zhejiang).

Ranjith et al. (2020) Zootaxa 4786 (3): 396-408.

Paroligoneurus songyangensis (He, 2000) 松阳 稀脉茧蜂 [New Combination]

Oligoneurus songyangensis He, 2000 松阳寡脉 茧蜂

Distribution: CHINA (Zhejiang).

Ranjith et al. (2020) Zootaxa 4786 (3): 396-408.

Elasmosoma Ruthe, 1858

Synonym: Sinoneoneurus He, Chen \& van Achterberg, 1997 [New Synonym]

Distribution: Palaearctic, Nearctic and Oriental Regions.

Li et al. (2020) Zoological Systematics 45(4): 281-289. 
Ectemnoplax peruliventris Enderlein, 1920 白腹 埃独蜂

Synonym: Ectemnoplax xizanginensis Wang, Chen \& He, 2006 [New Synonym]

Distribution: CHINA (Hainan, Taiwan, Xizang); Japan.

Li, He \& Chen (2020) Zootaxa 4818(1): 1-74.

\section{Ichneumonidae 姬蜂科}

Agrothereutes aprocerius Sheng, Li \& Sun, 2020

叶蜂田猎姬蜂 [New Species]

Holotype 9 (GSFGPM).

Host: Aproceros maculatus Wei 斑背近脉三节 叶蜂.

Distribution: CHINA (Hebei).

Sheng, Sun \& Li (2020) Ichneumonids parasitizing sawflies in China (Hymenoptera, Ichneumonidae). Science Press, Beijing. (in Chinese with English abstract)

Alexeter albimaculatus Sheng, Sun \& Li, 2020 白 斑亚力姬蜂 [New Species]

Holotype $q$ (GSFGPM).

Host: Unknown.

Distribution: CHINA (Ningxia).

Sheng, Sun \& Li (2020) Ichneumonids parasitizing sawflies in China (Hymenoptera, Ichneumonidae). Science Press, Beijing. (in Chinese with English abstract)

Alexeter zangicus Sheng, Sun \& Li, 2020 藏亚力 姬蜂 [New Species]

Holotype $q$ (GSFGPM).

Host: Unknown.

Distribution: CHINA (Tibet).
Sheng, Sun \& Li (2020) Ichneumonids parasitizing sawflies in China (Hymenoptera, Ichneumonidae). Science Press, Beijing. (in Chinese with English abstract)

Anoncus Townes, 1970 无凹姬蜂属 [New Record Genus]

Distribution: CHINA (Shandong).

Sheng, Sun \& Li (2020) Ichneumonids parasitizing sawflies in China (Hymenoptera, Ichneumonidae). Science Press, Beijing. (in Chinese with English abstract)

Anoncus maculatus Sheng, Sun \& Li, 2020 斑无 凹姬蜂 [New Species]

Holotype $q$ (GSFGPM).

Host: Unknown.

Distribution: CHINA (Shandong).

Sheng, Sun \& Li (2020) Ichneumonids parasitizing sawflies in China (Hymenoptera, Ichneumonidae). Science Press, Beijing. (in Chinese with English abstract)

Apophysius latus Pham, Matsumoto, Konishi, Sheng \& Broad, 2020 纵带多棘姬蜂 [New Speices]

Holotype $\bigcirc$ (IEBR).

Distribution: CHINA (Guangxi, Jiangxi, Yunnan). Pham et al. (2020) Zootaxa 4802(2): 301-316.

Azelus Förster, 1869 粮姬蜂属 [New Record Genus]

Distribution: CHINA (Shaanxi).

Sheng, Sun \& Li (2020) Ichneumonids parasitizing sawflies in China (Hymenoptera, Ichneumonidae). Science Press, Beijing. (in Chinese with English abstract) 
Azelus ankangicus Sheng, Sun \& Li, 2020 安康

粮姬蜂 [New Species]

Holotype $\odot$ (GSFGPM).

Host: Unknown.

Distribution: CHINA (Shaanxi).

Sheng, Sun \& Li (2020) Ichneumonids parasitizing sawflies in China (Hymenoptera, Ichneumonidae). Science Press, Beijing. (in Chinese with English abstract)

Bathythrix rufiscuta Sheng, Li \& Sun, 2020 红盾 泥甲姬蜂 [New Species]

Host: Pristiphora erichsonii (Hartig) 落叶松叶蜂.

Distribution: CHINA (Ningxia).

Sheng, Sun \& Li (2020) Ichneumonids parasitizing sawflies in China (Hymenoptera, Ichneumonidae). Science Press, Beijing. (in Chinese with English abstract)

Barytarbes motuoicus Sheng, Sun \& Li, 2020 墨 脱霸姬蜂 [New Species]

Holotype $q$ (GSFGPM).

Host: Unknown.

Distribution: CHINA (Tibet).

Sheng, Sun \& Li (2020) Ichneumonids parasitizing sawflies in China (Hymenoptera, Ichneumonidae). Science Press, Beijing. (in Chinese with English abstract)

Caenocryptus weiningicus Sheng, Li \& Sun, 2020 威宁雉唇姬蜂 [New Species]

Host: Neodiprion huizeensis Xiao \& Zhou 会泽新 松叶蜂.

Distribution: CHINA (Guizhou).

Sheng, Sun \& Li (2020) Ichneumonids parasitizing sawflies in China (Hymenoptera,
Ichneumonidae). Science Press, Beijing. (in

Chinese with English abstract)

Campodorus albilineatus Sheng, Sun \& Li, 2020

白纹堪姬蜂 [New Species]

Holotype + (GSFGPM).

Host: Unknown.

Distribution: CHINA (Guangxi).

Sun et al. (2020) European Journal of

Taxonomy (658).

Campodorus punctatus Sheng, Sun \& Li, 2020 点 堪姬蜂 [New Species]

Holotype $\odot$ (GSFGPM).

Host: Unknown.

Distribution: CHINA (Beijing).

Sun et al. (2020) European Journal of Taxonomy (658).

Campodorus rasilis Sheng, Sun \& Li, 2020 光堪 姬蜂 [New Species]

Holotype $q$ (GSFGPM).

Host: Unknown.

Distribution: CHINA (Beijing).

Sun et al. (2020) European Journal of Taxonomy (658).

Campodorus shandongicus Sheng, Sun \& Li, 2020 山东堪姬蜂 [New Species]

Holotype 9 (GSFGPM).

Host: Unknown.

Distribution: CHINA (Shandong).

Sun et al. (2020) European Journal of Taxonomy (658).

Campodorus truncatus Sheng, Sun \& Li, 2020 截 堪姬蜂 [New Species] 
Holotype $\odot$ (GSFGPM).

Host: Unknown.

Distribution: CHINA (Liaoning).

Sun et al. (2020) European Journal of

Taxonomy (658).

Campoletis deserticola Sheng \& Zhou, 2020 沙

齿唇姬蜂 [New Species]

Holotype + (GSFGPM).

Host: Unknown.

Distribution: CHINA (Beijing, Liaoning).

Wei et al. (2020) ZooKeys 1004: 99-108.

Casinaria coloratilis Vas, 2020 暗足凹眼姬蜂

[New Species]

Holotype $q$ (MZLU).

Host: Unknown.

Distribution: CHINA (Taiwan).

Vas (2020) Opuscula Zoologica Budapest 51(2): 133-152.

Casinaria russea Vas, 2020 红腹凹眼姬蜂 [New

Species]

Holotype $q$ (MZLU).

Host: Unknown.

Distribution: CHINA (Taiwan).

Vas (2020) Opuscula Zoologica Budapest 51(2): 133-152.

Casinaria vesca Vas, 2020 纤细凹眼姬蜂 [New Species]

Holotype $q$ (MZLU).

Host: Unknown.

Distribution: CHINA (Taiwan).

Vas (2020) Opuscula Zoologica Budapest 51(2): 133-152.
Charops aeruginosus Vas, 2020 铜色悬茧姬蜂

[New Species]

Holotype + (HNHM).

Host: Unknown.

Distribution: CHINA (Taiwan).

Vas (2020) Acta Zoologica Academiae

Scientiarum Hungaricae 66(3): 247-267.

Diadegma neodiprionis Sheng, Li \& Sun, 2020 叶 蜂弯尾姬蜂 [New Species]

Holotype $q$ (GSFGPM).

Host: Neodiprion huizeensis Xiao \& Zhou 会泽新 松叶蜂.

Distribution: CHINA (Guizhou).

Sheng, Sun \& Li (2020) Ichneumonids parasitizing sawflies in China (Hymenoptera, Ichneumonidae). Science Press, Beijing. (in Chinese with English abstract)

Cosmoconus chuanicus Sheng, Sun \& Li, 2020 川 角姬蜂 [New Species]

Holotype $q$ (GSFGPM).

Host: Unknown.

Distribution: CHINA (Sichuan).

Sheng, Sun \& Li (2020) Ichneumonids parasitizing sawflies in China (Hymenoptera, Ichneumonidae). Science Press, Beijing. (in Chinese with English abstract)

Cosmoconus truncatus Sheng, Sun \& Li, 2020 截 角姬蜂 [New Species]

Holotype $q$ (GSFGPM).

Host: Unknown.

Distribution: CHINA (Beijing, Sichuan).

Sheng, Sun \& Li (2020) Ichneumonids parasitizing sawflies in China (Hymenoptera, 
Ichneumonidae). Science Press, Beijing. (in

Chinese with English abstract)

Cosmoconus zongi Sheng, Sun \& Li, 2020 宗角

姬蜂 [New Species]

Holotype $\odot$ (GSFGPM).

Host: Unknown.

Distribution: CHINA (Beijing).

Sheng, Sun \& Li (2020) Ichneumonids parasitizing sawflies in China (Hymenoptera, Ichneumonidae). Science Press, Beijing. (in Chinese with English abstract)

Cycasis Townes, 1965 迷姬蜂属 [New Record Genus]

Distribution: CHINA (Jilin).

Sheng, Sun \& Li (2020) Ichneumonids parasitizing sawflies in China (Hymenoptera, Ichneumonidae). Science Press, Beijing. (in Chinese with English abstract)

Cycasis jilinica Sheng, Sun \& Li, 2020 吉林迷姬 蜂 [New Species]

Holotype $\odot$ (GSFGPM).

Host: Unknown.

Distribution: CHINA (Jilin).

Sheng, Sun \& Li (2020) Ichneumonids parasitizing sawflies in China (Hymenoptera, Ichneumonidae). Science Press, Beijing. (in Chinese with English abstract)

Distathma Townes，1970 墨线姬蜂属 [New Record Genus]

Distribution: CHINA (Ningxia).

Sheng, Sun \& Li (2020) Ichneumonids parasitizing sawflies in China (Hymenoptera, Ichneumonidae). Science Press, Beijing. (in Chinese with English abstract)
Distathma ningxiaica Sheng, Li \& Sun, 2020 宁 墨线姬蜂 [New Species]

Holotype 9 (GSFGPM).

Host: Pristiphora erichsonii (Hartig) 落叶松叶蜂. Distribution: CHINA (Ningxia).

Sheng, Sun \& Li (2020) Ichneumonids parasitizing sawflies in China (Hymenoptera, Ichneumonidae). Science Press, Beijing. (in Chinese with English abstract)

Endasys pristiphorae Sheng, 2020 叶蜂恩姬蜂 [New Species]

Holotype + (GSFGPM).

Host: Stauronematus compresicornis (Fabricius) 杨直角叶蜂, Pristiphora xibei Wei \& Xia 西北 槌缘叶蜂, Pristiphora erichsonii (Hartig) 落叶 松叶蜂.

Distribution: CHINA (Shandong, Shaanxi, Ningxia, Gansu).

Duan et al. (2020) Zootaxa 4743(1): 111-118.

Holotype $q$ (GSFGPM).

Endasys proteuclastae Sheng, Sun \& Li, 2020 螟 恩姬蜂 [New Species]

Holotype + (GSFGPM).

Host: Proteuclasta stotzeneri (Caradja) 旱柳原叶 螟.

Distribution: CHINA (Ningxia, Shandong).

Sheng, Sun \& Li (2020) Ichneumonids parasitizing sawflies in China (Hymenoptera, Ichneumonidae). Science Press, Beijing. (in Chinese with English abstract)

Enytus Cameron, 1905 尚姬蜂属 [New Record Genus] 
Distribution: CHINA (Jiangxi, Jilin); Mongolia, Russia, Europe, South Africa, Sri Lanka.

Sheng, Sun \& Li (2020) Ichneumonids parasitizing sawflies in China (Hymenoptera, Ichneumonidae). Science Press, Beijing. (in Chinese with English abstract)

Enytus ganicus Sheng, Sun \& Li, 2020 赣尚姬蜂

[New Species]

Holotype $\odot$ (GSFGPM).

Host: Unknown.

Distribution: CHINA (Jiangxi).

Sheng, Sun \& Li (2020) Ichneumonids parasitizing sawflies in China (Hymenoptera, Ichneumonidae). Science Press, Beijing. (in Chinese with English abstract)

Eridolius beijingicus Sheng, Sun \& Li, 2020 京鼓 姬蜂 [New Species]

Holotype 9 (GSFGPM).

Host: Unknown.

Distribution: CHINA (Beijing).

Sheng, Sun \& Li (2020) Ichneumonids parasitizing sawflies in China (Hymenoptera, Ichneumonidae). Science Press, Beijing. (in Chinese with English abstract)

Euryproctus wugongensis Sheng, Sun \& Li, 2020 武功阔肛姬蜂 [New Species]

Holotype 9 (GSFGPM).

Host: Unknown.

Distribution: CHINA (Jiangxi).

Sheng, Sun \& Li (2020) Ichneumonids parasitizing sawflies in China (Hymenoptera, Ichneumonidae). Science Press, Beijing. (in Chinese with English abstract)
Euryproctus zongi Sheng, Sun \& Li, 2020 宗氏阔 肛姬蜂 [New Species]

Holotype $q$ (GSFGPM).

Host: Unknown.

Distribution: CHINA (Beijing).

Sheng, Sun \& Li (2020) Ichneumonids parasitizing sawflies in China (Hymenoptera, Ichneumonidae). Science Press, Beijing. (in Chinese with English abstract)

Exyston lineatus Sheng, Li \& Sun, 2020 纹易刻 姬蜂 [New Species]

Holotype $q$ (GSFGPM).

Host: Neodiprion huizeensis Xiao \& Zhou会泽 新松叶蜂.

Distribution: CHINA (Guizhou).

Sheng, Sun \& Li (2020) Ichneumonids parasitizing sawflies in China (Hymenoptera, Ichneumonidae). Science Press, Beijing. (in Chinese with English abstract)

Exyston xizangicus Sheng, Li \& Sun, 2020 西藏 易刻姬蜂 [New Species]

Holotype $\odot$ (GSFGPM).

Host: Unknown.

Distribution: CHINA (Tibet).

Sheng, Sun \& Li (2020) Ichneumonids parasitizing sawflies in China (Hymenoptera, Ichneumonidae). Science Press, Beijing. (in Chinese with English abstract)

Gelis weiningicus Sheng, Li \& Sun, 2020 威宁沟 姬蜂 [New Species]

Holotype $q$ (GSFGPM).

Host: Neodiprion huizeensis Xiao \& Zhou 会泽新 松叶蜂.

Distribution: CHINA (Guizhou). 
Sheng, Sun \& Li (2020) Ichneumonids parasitizing sawflies in China (Hymenoptera, Ichneumonidae). Science Press, Beijing. (in Chinese with English abstract)

Glyphicnemis qinica Sheng, Li \& Sun, 2020 秦离 距姬蜂 [New Species]

Holotype + (GSFGPM).

Host: Unknown.

Distribution: CHINA (Shaanxi).

Sheng, Sun \& Li (2020) Ichneumonids parasitizing sawflies in China (Hymenoptera, Ichneumonidae). Science Press, Beijing. (in Chinese with English abstract)

Goryphus pristiphorae Sheng, Li \& Sun, 2020 叶 蜂驼姬蜂 [New Species]

Holotype $\odot$ (GSFGPM).

Host: Pristiphora erichsonii (Hartig) 落叶松叶蜂. Distribution: CHINA (Gansu).

Sheng, Sun \& Li (2020) Ichneumonids parasitizing sawflies in China (Hymenoptera, Ichneumonidae). Science Press, Beijing. (in Chinese with English abstract)

Goryphus weiningicus Sheng, Li \& Sun, 2020 威 宁驼姬蜂 [New Species]

Holotype $\odot$ (GSFGPM).

Host: Neodiprion huizeensis Xiao \& Zhou 会泽新 松叶蜂.

Distribution: CHINA (Guizhou).

Sheng, Sun \& Li (2020) Ichneumonids parasitizing sawflies in China (Hymenoptera, Ichneumonidae). Science Press, Beijing. (in Chinese with English abstract)
Homaspis sichuanica Sheng, Sun \& Li, 2020 川 拟姬蜂 [New Species]

Holotype + (GSFGPM).

Host: Unknown.

Distribution: CHINA (Sichuan).

Sheng, Sun \& Li (2020) Ichneumonids parasitizing sawflies in China (Hymenoptera, Ichneumonidae). Science Press, Beijing. (in Chinese with English abstract)

Kristotomus brevis Sheng, Sun \& Li, 2020 短克 里姬蜂 [New Species]

Holotype $\circ$ (GSFGPM).

Host: Unknown.

Distribution: CHINA (Jiangxi).

Sheng, Sun \& Li (2020) Ichneumonids parasitizing sawflies in China (Hymenoptera, Ichneumonidae). Science Press, Beijing. (in Chinese with English abstract)

Lagarotis Förster, 1869 拉加姬蜂属 [New Record Genus]

Distribution: CHINA (Beijing, Jiangxi).

Sheng, Sun \& Li (2020) Ichneumonids parasitizing sawflies in China (Hymenoptera, Ichneumonidae). Science Press, Beijing. (in Chinese with English abstract)

Lagarotis beijingensis Sheng, Sun \& Li, 2020 京 拉加姬蜂 [New Species]

Holotype + (GSFGPM).

Host: Unknown.

Distribution: CHINA (Beijing).

Sheng, Sun \& Li (2020) Ichneumonids parasitizing sawflies in China (Hymenoptera, Ichneumonidae). Science Press, Beijing. (in Chinese with English abstract) 
Lagarotis ganica Sheng, Sun \& Li, 2020 赣拉加 姬蜂 [New Species]

Holotype + (GSFGPM).

Host: Unknown.

Distribution: CHINA (Jiangxi).

Sheng, Sun \& Li (2020) Ichneumonids parasitizing sawflies in China (Hymenoptera, Ichneumonidae). Science Press, Beijing. (in Chinese with English abstract)

Lamachus dilatatus Sheng, Li \& Sun, 2020 阔侵 姬蜂 [New Species]

Holotype + (GSFGPM).

Host: Unknown.

Distribution: CHINA (Shandong).

Sheng, Sun \& Li (2020) Ichneumonids parasitizing sawflies in China (Hymenoptera, Ichneumonidae). Science Press, Beijing. (in Chinese with English abstract)

Mastrus luicus Sheng, Sun \& Li, 2020 鲁搜姬蜂

[New Species]

Holotype $\odot$ (GSFGPM).

Host: Unknown.

Distribution: CHINA (Shandong).

Sheng, Sun \& Li (2020) Ichneumonids parasitizing sawflies in China (Hymenoptera, Ichneumonidae). Science Press, Beijing. (in Chinese with English abstract)

Mesoleptidea maculata Sheng, Sun \& Li, 2020 斑 长颚姬蜂 [New Species]

Holotype $q$ (GSFGPM).

Host: Unknown.

Distribution: CHINA (Shaanxi).
Sheng, Sun \& Li (2020) Ichneumonids parasitizing sawflies in China (Hymenoptera, Ichneumonidae). Science Press, Beijing. (in Chinese with English abstract)

Microstenus rufithorax Sheng, Li \& Sun, 2020 褐 胸纵沟姬蜂 [New Species]

Holotype + (GSFGPM).

Host: Unknown.

Distribution: CHINA (Guangxi).

Li, Sun \& Sheng (2020) Zootaxa 4838: 594-600.

Nemeritis Holmgren, 1860 呐姬蜂属 [New Record Genus]

Distribution: CHINA (Guizhou, Beijing).

Sheng, Sun \& Li (2020) Ichneumonids parasitizing sawflies in China (Hymenoptera, Ichneumonidae). Science Press, Beijing. (in Chinese with English abstract)

Nemeritis niger Sheng, Li \& Sun, 2020 黑呐姬蜂 [New Species]

Holotype $\odot$ (GSFGPM).

Host: Unknown.

Distribution: CHINA (Beijing).

Sheng, Sun \& Li (2020) Ichneumonids parasitizing sawflies in China (Hymenoptera, Ichneumonidae). Science Press, Beijing. (in Chinese with English abstract)

Nemeritis pilosa Sheng, Li \& Sun, 2020 毛呐姬 蜂 [New Species]

Holotype 9 (GSFGPM).

Host: Neodiprion sp. 新松叶蜂.

Distribution: CHINA (Guizhou).

Sheng, Sun \& Li (2020) Ichneumonids parasitizing sawflies in China (Hymenoptera, 
Ichneumonidae). Science Press, Beijing. (in

Chinese with English abstract)

Olesicampe albibasalis Sheng, Li \& Sun, 2020 白

基除蠋姬蜂 [New Species]

Holotype $\odot$ (GSFGPM).

Host: Gilpinia tabulaeformis Xiao 油松吉松叶蜂.

Distribution: CHINA (Gansu).

Sheng, Sun \& Li (2020) Ichneumonids parasitizing sawflies in China (Hymenoptera, Ichneumonidae). Science Press, Beijing. (in Chinese with English abstract)

Olesicampe jingyuanensis Sheng, Li \& Sun, 2020 靖远除蠋姬蜂 [New Species]

Holotype $\odot$ (GSFGPM).

Host: Diprion jingyuanensis Xiao \& Zhang 靖远 松叶蜂.

Distribution: CHINA (Gansu).

Sheng, Sun \& Li (2020) Ichneumonids parasitizing sawflies in China (Hymenoptera, Ichneumonidae). Science Press, Beijing. (in Chinese with English abstract)

Olesicampe melana Sheng, Li \& Sun, 2020 黑除 蠋姬蜂 [New Species]

Holotype $q$ (GSFGPM).

Host: Pristiphora erichsonii (Hartig) 落叶松叶蜂.

Distribution: CHINA (Heilongjiang).

Sheng, Sun \& Li (2020) Ichneumonids parasitizing sawflies in China (Hymenoptera, Ichneumonidae). Science Press, Beijing. (in Chinese with English abstract)

Olesicampe populnea Sheng, Li \& Sun, 2020 杨 除蠋姬蜂 [New Species]

Holotype \& (GSFGPM).
Host: Pristiphora beijingensis Zhou \&Zhang 北 京杨锉叶蜂.

Distribution: CHINA (Liaoning).

Sheng, Sun \& Li (2020) Ichneumonids parasitizing sawflies in China (Hymenoptera, Ichneumonidae). Science Press, Beijing. (in Chinese with English abstract)

Orthocentrus caudalis Humala \& Lee, 2020 长尾 拱脸姬蜂 [New Species]

Holotype $q$ (DNUE).

Host: Unknown.

Distribution: CHINA (Jilin); South Korea.

Humala, Lee \& Choi (2020) Journal of Hymenoptera Research 75: 15-65.

Phobetes convavus Sheng, Sun \& Li, 2020 凹浮 姬蜂 [New Species]

Holotype 9 (GSFGPM).

Host: Unknown.

Distribution: CHINA (Jiangxi).

Sheng, Sun \& Li (2020) Ichneumonids parasitizing sawflies in China (Hymenoptera, Ichneumonidae). Science Press, Beijing. (in Chinese with English abstract)

Phobetes ruficoxalis Sheng, Sun \& Li, 2020 红基 浮姬蜂 [New Species]

Holotype $\odot$ (GSFGPM).

Host: Unknown.

Distribution: CHINA (Jiangxi).

Sheng, Sun \& Li (2020) Ichneumonids parasitizing sawflies in China (Hymenoptera, Ichneumonidae). Science Press, Beijing. (in Chinese with English abstract) 
Phygadeuon yanjiensis Sheng, Li \& Sun, 2020 延

吉粗角姬蜂 [New Species]

Holotype $\widehat{\partial}$ (GSFGPM).

Host: Cleptes semiauratus (L.) 暗尖胸青蜂.

Distribution: CHINA (Jilin).

Sheng, Sun \& Li (2020) Ichneumonids parasitizing sawflies in China (Hymenoptera, Ichneumonidae). Science Press, Beijing. (in Chinese with English abstract)

Phytodietus (Phytodietus) xui Kostro-Ambroziak \& Reshchikov, 2020 许氏短梳姬蜂 [New Species]

Holotype $q$ (KIZ).

Host: Unknown.

Distribution: CHINA (Yunnan).

Kostro-Ambroziak, Zhou \& Reshchikov (2020)

Zootaxa 4877(2): 391-395.

Pleolophus rugulosus Sheng, Sun \& Li, 2020 皱 瘤角姬蜂 [New Species]

Holotype 9 (GSFGPM).

Host: Pristiphora erichsonii (Hartig) 落叶松叶蜂、 Neodiprion fengningensis Xiao \& Zhou 丰宁新松 叶蜂.

Distribution: CHINA (Shanxi, Gansu).

Sheng, Sun \& Li (2020) Ichneumonids parasitizing sawflies in China (Hymenoptera, Ichneumonidae). Science Press, Beijing. (in Chinese with English abstract)

Protarchus Förster, 1868 前姬蜂属 [New Record Genus]

Distribution: CHINA (Beijing).

Sheng, Sun \& Li (2020) Ichneumonids parasitizing sawflies in China (Hymenoptera,
Ichneumonidae). Science Press, Beijing. (in

Chinese with English abstract)

Protarchus maculatus Sheng, Sun \& Li, 2020 斑 前姬蜂 [New Species]

Holotype \& (GSFGPM).

Host: Unknown.

Distribution: CHINA (Beijing).

Sheng, Sun \& Li (2020) Ichneumonids parasitizing sawflies in China (Hymenoptera, Ichneumonidae). Science Press, Beijing. (in Chinese with English abstract)

Perilissus incarinatus Sheng, Sun \& Li, 2020 缺 脊波姬蜂 [New Species]

Holotype $q$ (GSFGPM).

Host: Unknown.

Distribution: CHINA (Guizhou).

Sheng, Sun \& Li (2020) Ichneumonids parasitizing sawflies in China (Hymenoptera, Ichneumonidae). Science Press, Beijing. (in Chinese with English abstract)

Lethades wugongensis Sheng, Sun \& Li, 2020 武 功失姬蜂 [New Species]

Holotype + (GSFGPM).

Host: Unknown.

Distribution: CHINA (Jiangxi).

Sheng, Sun \& Li (2020) Ichneumonids parasitizing sawflies in China (Hymenoptera, Ichneumonidae). Science Press, Beijing. (in Chinese with English abstract)

Rhinotorus nigrus Sheng, Li \& Sun, 2020 黑犀姬 蜂 [New Species]

Holotype $\odot$ (GSFGPM).

Host: Pristiphora erichsonii (Hartig) 落叶松叶蜂. 
Distribution: CHINA (Jilin).

Li, Sun \& Sheng (2020) Journal of Hymenoptera

Research 77: 203-211.

Rhorus bimaculatus Sheng, Sun \& Li, 2020 双斑

壮姬蜂 [New Species]

Holotype ${ }_{+}$(GSFGPM).

Host: Unknown.

Distribution: CHINA (Liaoning).

Sheng, Sun \& Li (2020) Ichneumonids parasitizing sawflies in China (Hymenoptera, Ichneumonidae). Science Press, Beijing. (in Chinese with English abstract)

Rhorus carinatus Sheng, Sun \& Li, 2020 脊壮姬 蜂 [New Species]

Holotype $\odot$ (GSFGPM).

Host: Unknown.

Distribution: CHINA (Tibet).

Sheng, Sun \& Li (2020) Ichneumonids parasitizing sawflies in China (Hymenoptera, Ichneumonidae). Science Press, Beijing. (in Chinese with English abstract)

Rhorus concavus Sheng, Sun \& Li, 2020 端凹壮 姬蜂 [New Species]

Holotype $q$ (GSFGPM).

Host: Unknown.

Distribution: CHINA (Jiangxi).

Sheng, Sun \& Li (2020) Ichneumonids parasitizing sawflies in China (Hymenoptera, Ichneumonidae). Science Press, Beijing. (in Chinese with English abstract)

Rhorus denticlypealis Sheng, Sun \& Li, 2020 齿 唇壮姬蜂 [New Species]

Holotype + (GSFGPM).
Host: Unknown.

Distribution: CHINA (Jiangxi).

Sheng, Sun \& Li (2020) Ichneumonids parasitizing sawflies in China (Hymenoptera, Ichneumonidae). Science Press, Beijing. (in Chinese with English abstract)

Rhorus facialis Sheng, Sun \& Li, 2020 颜壮姬蜂

[New Species]

Holotype $\odot$ (GSFGPM).

Host: Unknown.

Distribution: CHINA (Liaoning).

Sheng, Sun \& Li (2020) Ichneumonids parasitizing sawflies in China (Hymenoptera, Ichneumonidae). Science Press, Beijing. (in Chinese with English abstract)

Rhorus flavofacialis Sheng, Sun \& Li, 2020 黄颜 壮姬蜂 [New Species]

Holotype ${ }_{+}$(GSFGPM).

Host: Pristiphora erichsonii (Hartig) 落叶松叶蜂. Distribution: CHINA (Ningxia, Gansu).

Sheng, Sun \& Li (2020) Ichneumonids parasitizing sawflies in China (Hymenoptera, Ichneumonidae). Science Press, Beijing. (in Chinese with English abstract)

Rhorus flavus Sheng, Sun \& Li, 2020 黄壮姬蜂 [New Species]

Holotype $q$ (GSFGPM).

Host: Unknown.

Distribution: CHINA (Beijing).

Sheng, Sun \& Li (2020) Ichneumonids parasitizing sawflies in China (Hymenoptera, Ichneumonidae). Science Press, Beijing. (in Chinese with English abstract) 
Rhorus huinanicus Sheng, Sun \& Li, 2020 辉南

壮姬蜂 [New Species]

Holotype + (GSFGPM).

Host: Unknown.

Distribution: CHINA (Jilin, Liaoning).

Sheng, Sun \& Li (2020) Ichneumonids parasitizing sawflies in China (Hymenoptera, Ichneumonidae). Science Press, Beijing. (in Chinese with English abstract)

Rhorus liaoensis Sheng, Sun \& Li, 2020 辽壮姬 蜂 [New Species]

Holotype $q$ (GSFGPM).

Host: Unknown.

Distribution: CHINA (Liaoning).

Sheng, Sun \& Li (2020) Ichneumonids parasitizing sawflies in China (Hymenoptera, Ichneumonidae). Science Press, Beijing. (in Chinese with English abstract)

Rhorus lishuicus Sheng, Sun \& Li, 2020 丽水壮 姬蜂 [New Species]

Holotype $\odot$ (GSFGPM).

Host: Unknown.

Distribution: CHINA (Zhejiang).

Sheng, Sun \& Li (2020) Ichneumonids parasitizing sawflies in China (Hymenoptera, Ichneumonidae). Science Press, Beijing. (in Chinese with English abstract)

Rhorus mandibularis Sheng, Sun \& Li, 2020 颚 壮姬蜂 [New Species]

Holotype $\hat{\partial}$ (GSFGPM).

Host: Unknown.

Distribution: CHINA (Jiangxi).

Sheng, Sun \& Li (2020) Ichneumonids parasitizing sawflies in China (Hymenoptera,
Ichneumonidae). Science Press, Beijing. (in

Chinese with English abstract)

Rhorus melanus Sheng, Sun \& Li, 2020 黑 壮 姬蜂 [New Species]

Holotype + (GSFGPM).

Host: Unknown.

Distribution: CHINA (Tibet).

Sheng, Sun \& Li (2020) Ichneumonids parasitizing sawflies in China (Hymenoptera, Ichneumonidae). Science Press, Beijing. (in Chinese with English abstract)

Rhorus nigriantenntus Sheng, Sun \& Li, 2020 黑 角壮姬蜂 [New Species]

Holotype + (GSFGPM).

Host: Unknown.

Distribution: CHINA (Beijing, Jiangxi).

Sheng, Sun \& Li (2020) Ichneumonids parasitizing sawflies in China (Hymenoptera, Ichneumonidae). Science Press, Beijing. (in Chinese with English abstract)

Rhorus nigriclypealis Sheng, Sun \& Li, 2020 黑 唇壮姬蜂 [New Species]

Holotype 9 (GSFGPM).

Host: Unknown.

Distribution: CHINA (Liaoning).

Sheng, Sun \& Li (2020) Ichneumonids parasitizing sawflies in China (Hymenoptera, Ichneumonidae). Science Press, Beijing. (in Chinese with English abstract)

Rhorus nigripedalis Sheng, Sun \& Li, 2020 黑足 壮姬蜂 [New Species]

Holotype + (GSFGPM).

Host: Unknown. 
Distribution: CHINA (Tibet, Sichuan).

Sheng, Sun \& Li (2020) Ichneumonids parasitizing sawflies in China (Hymenoptera, Ichneumonidae). Science Press, Beijing. (in Chinese with English abstract)

Rhorus petiolatus Sheng, Sun \& Li, 2020 柄壮姬 蜂 [New Species]

Holotype + (GSFGPM).

Host: Unknown.

Distribution: CHINA (Jiangxi).

Sheng, Sun \& Li (2020) Ichneumonids parasitizing sawflies in China (Hymenoptera, Ichneumonidae). Science Press, Beijing. (in Chinese with English abstract)

Rhorus recavus Sheng, Sun \& Li, 2020 凹唇壮姬 蜂 [New Species]

Holotype 9 (GSFGPM).

Host: Unknown.

Distribution: CHINA (Jiangxi).

Sheng, Sun \& Li (2020) Ichneumonids parasitizing sawflies in China (Hymenoptera, Ichneumonidae). Science Press, Beijing. (in Chinese with English abstract)

Rhorus urceolatus Sheng, Sun \& Li, 2020 瓶壮姬 蜂 [New Species]

Holotype 9 (GSFGPM).

Host: Unknown.

Distribution: CHINA (Jiangxi).

Sheng, Sun \& Li (2020) Ichneumonids parasitizing sawflies in China (Hymenoptera, Ichneumonidae). Science Press, Beijing. (in Chinese with English abstract)
Scambus qinghaiicus Sheng, Sun \& Li, 2020 青 海曲姬蜂 [New Species]

Holotype $\odot$ (GSFGPM).

Host: Pontania sp.掼叶蜂.

Distribution: CHINA (Qinghai).

Sheng, Sun \& Li (2020) Ichneumonids parasitizing sawflies in China (Hymenoptera, Ichneumonidae). Science Press, Beijing. (in Chinese with English abstract)

Scolobates argeae Sheng, Sun \& Li, 2020 叶蜂齿 胫姬蜂 [New Species]

Holotype $P$ (GSFGPM).

Host: Unknown.

Distribution: CHINA (Gansu, Heilongjinag, Jilin, Ningxia).

Sheng, Sun \& Li (2020) Ichneumonids parasitizing sawflies in China (Hymenoptera, Ichneumonidae). Science Press, Beijing. (in Chinese with English abstract)

Scolobates fulvus Sheng, Sun \& Li, 2020 黄齿胫 姬蜂 [New Species]

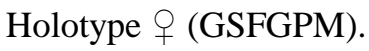

Host: Unknown.

Distribution: CHINA (Liaoning).

Sheng, Sun \& Li (2020) Ichneumonids parasitizing sawflies in China (Hymenoptera, Ichneumonidae). Science Press, Beijing. (in Chinese with English abstract)

Scolobates maculatus Sheng, Sun \& Li, 2020 斑 齿胫姬蜂 [New Species]

Holotype $\odot$ (GSFGPM).

Host: Unknown.

Distribution: CHINA (Liaoning). 
Sheng, Sun \& Li (2020) Ichneumonids parasitizing sawflies in China (Hymenoptera, Ichneumonidae). Science Press, Beijing. (in Chinese with English abstract)

Scolobates oppositus Sheng, Sun \& Li, 2020 对 脉齿胫姬蜂 [New Species]

Holotype $\widehat{\partial}$ (GSFGPM).

Host: Unknown.

Distribution: CHINA (Tibet, Sichuan).

Sheng, Sun \& Li (2020) Ichneumonids parasitizing sawflies in China (Hymenoptera, Ichneumonidae). Science Press, Beijing. (in Chinese with English abstract)

Scolobates parallelis Sheng, Sun \& Li, 2020 平 齿胫姬蜂 [New Species]

Holotype $\odot$ (GSFGPM).

Host: Unknown.

Distribution: CHINA (Jiangxi, Jilin).

Sheng, Sun \& Li (2020) Ichneumonids parasitizing sawflies in China (Hymenoptera, Ichneumonidae). Science Press, Beijing. (in Chinese with English abstract)

Scolobates rufiabdominalis Sheng, Sun \& $\mathrm{Li}$, 2020 褐腹齿胫姬蜂 [New Species]

Holotype 9 (GSFGPM).

Host: Unknown.

Distribution: CHINA (Hebei).

Sheng, Sun \& Li (2020) Ichneumonids parasitizing sawflies in China (Hymenoptera, Ichneumonidae). Science Press, Beijing. (in Chinese with English abstract)

Scolobates shinicus Sheng, Sun \& Li, 2020 亮齿 胫姬蜂 [New Species]
Holotype + (GSFGPM).

Host: Unknown.

Distribution: CHINA (Liaoning).

Sheng, Sun \& Li (2020) Ichneumonids parasitizing sawflies in China (Hymenoptera, Ichneumonidae). Science Press, Beijing. (in Chinese with English abstract)

Scolobates tergitalis Sheng, Sun \& Li, 2020 节齿 胫姬蜂 [New Species]

Holotype $q$ (GSFGPM).

Host: Unknown.

Distribution: CHINA (Fujian, Shaanxi).

Sheng, Sun \& Li (2020) Ichneumonids parasitizing sawflies in China (Hymenoptera, Ichneumonidae). Science Press, Beijing. (in Chinese with English abstract)

Scolobates trapezius Sheng, Sun \& Li, 2020 梯齿 胫姬蜂 [New Species]

Holotype $\odot$ (GSFGPM).

Host: Unknown.

Distribution: CHINA (Beijing, Sichuan, Ningxia). Sheng, Sun \& Li (2020) Ichneumonids parasitizing sawflies in China (Hymenoptera, Ichneumonidae). Science Press, Beijing. (in Chinese with English abstract)

Smicroplectrus Thomson, 1883 联姬蜂属 [New Record Genus]

Distribution: CHINA (Beijing).

Sheng, Sun \& Li (2020) Ichneumonids parasitizing sawflies in China (Hymenoptera, Ichneumonidae). Science Press, Beijing. (in Chinese with English abstract)

Smicroplectrus salixis Sheng, Li \& Sun, 2020 柳 联姬蜂 [New Species] 
Holotype $\odot$ (GSFGPM).

Host: Unknown.

Distribution: CHINA (Beijing).

Sheng, Sun \& Li (2020) Ichneumonids parasitizing sawflies in China (Hymenoptera, Ichneumonidae). Science Press, Beijing. (in Chinese with English abstract)

Sympherta benxica Sheng, Sun \& Li, 2020 本溪 利姬蜂 [New Species]

Holotype $q$ (GSFGPM).

Host: Unknown.

Distribution: CHINA (Liaoning).

Sheng, Sun \& Li (2020) Ichneumonids parasitizing sawflies in China (Hymenoptera, Ichneumonidae). Science Press, Beijing. (in Chinese with English abstract)

Sympherta linzhiica Sheng, Sun \& Li, 2020 林芝 利姬蜂 [New Species]

Holotype ${ }_{+}$(GSFGPM).

Host: Unknown.

Distribution: CHINA (Tibet).

Sheng, Sun \& Li (2020) Ichneumonids parasitizing sawflies in China (Hymenoptera, Ichneumonidae). Science Press, Beijing. (in Chinese with English abstract)

Sympherta motuoensis Sheng, Sun \& Li, 2020 墨 脱利姬蜂 [New Species]

Holotype ${ }_{+}$(GSFGPM).

Host: Unknown.

Distribution: CHINA (Tibet).

Sheng, Sun \& Li (2020) Ichneumonids parasitizing sawflies in China (Hymenoptera, Ichneumonidae). Science Press, Beijing. (in Chinese with English abstract)
Sympherta polycolor Sheng, Sun \& Li, 2020 多利 姬蜂 [New Species]

Holotype + (GSFGPM).

Host: Unknown.

Distribution: CHINA (Shaanxi).

Sheng, Sun \& Li (2020) Ichneumonids parasitizing sawflies in China (Hymenoptera, Ichneumonidae). Science Press, Beijing. (in Chinese with English abstract)

Syntactus niger Sheng, Sun \& Li, 2020 全黑合姬 蜂 [New Species]

Holotype ${ }_{+}$(GSFGPM).

Host: Unknown.

Distribution: CHINA (Jiangxi).

Sheng, Sun \& Li (2020) Ichneumonids parasitizing sawflies in China (Hymenoptera, Ichneumonidae). Science Press, Beijing. (in Chinese with English abstract)

Syntacuts rugosus Sheng, Sun \& Li, 2020 皱合姬 蜂 [New Species]

Holotype $q$ (GSFGPM).

Host: Unknown.

Distribution: CHINA (Jiangxi).

Sheng, Sun \& Li (2020) Ichneumonids parasitizing sawflies in China (Hymenoptera, Ichneumonidae). Science Press, Beijing. (in Chinese with English abstract)

Strongylopsis propodealis Sheng \& Sun, 2020 并 胸实姬蜂 [New Species]

Holotype $\odot$ (GSFGPM).

Host: Unknown.

Distribution: CHINA (Beijing).

Sun et al. (2020) Journal of Hymenoptera

Research 79: 1-13. 
Strongylopsis punctata Sheng \& Sun, 2020 刻点

实姬蜂 [New Species]

Holotype $P$ (GSFGPM).

Host: Unknown.

Distribution: CHINA (Beijing).

Sun et al. (2020) Journal of Hymenoptera

Research 79: 1-13.

Xenoschesis (Polycinetis) melana Sheng, Sun \&

Li, 2020 黑跃姬蜂 [New Species]

Holotype $\circ$ (GSFGPM).

Host: Unknown.

Distribution: CHINA (Tibet).

Sheng, Sun \& Li (2020) Ichneumonids parasitizing sawflies in China (Hymenoptera, Ichneumonidae). Science Press, Beijing. (in Chinese with English abstract)

Absyrtus Holmgren, 1859 折脉姬蜂属 [New Record Genus]

Distribution: CHINA (Ningxia, Beijing).

Sheng, Sun \& Li (2020) Ichneumonids parasitizing sawflies in China (Hymenoptera, Ichneumonidae). Science Press, Beijing. (in Chinese with English abstract)

Absyrtus vicinator (Thunberg, 1822) 邻折脉姬蜂 [New Record]

Distribution: CHINA (Ningxia, Beijing).

Sheng, Sun \& Li (2020) Ichneumonids parasitizing sawflies in China (Hymenoptera, Ichneumonidae). Science Press, Beijing. (in Chinese with English abstract)

Acrotomus Holmgren, 1857 平唇姬蜂属 [New Record Genus]

Distribution: CHINA (Liaoning, Jilin).
Sheng, Sun \& Li (2020) Ichneumonids parasitizing sawflies in China (Hymenoptera, Ichneumonidae). Science Press, Beijing. (in Chinese with English abstract)

Acrotomus albidulus Kasparya, 1986 白平唇姬 蜂 [New Record]

Distribution: CHINA (Liaoning).

Sheng, Sun \& Li (2020) Ichneumonids parasitizing sawflies in China (Hymenoptera, Ichneumonidae). Science Press, Beijing. (in Chinese with English abstract)

Acrotomus lucidulus (Gravenhorst, 1829) 卢平唇 姬蜂 [New Record]

Distribution: CHINA (Jilin).

Sheng, Sun \& Li (2020) Ichneumonids parasitizing sawflies in China (Hymenoptera, Ichneumonidae). Science Press, Beijing. (in Chinese with English abstract)

Acrotomus succinctus (Gravenhorst, 1829) 速平 唇姬蜂 [New Record]

Distribution: CHINA (Liaoning).

Sheng, Sun \& Li (2020) Ichneumonids parasitizing sawflies in China (Hymenoptera, Ichneumonidae). Science Press, Beijing. (in Chinese with English abstract)

Alexeter fallax (Holmgren, 1857) 发亚力姬蜂 [New Record]

Distribution: CHINA (Ningxia).

Sheng, Sun \& Li (2020) Ichneumonids parasitizing sawflies in China (Hymenoptera, Ichneumonidae). Science Press, Beijing. (in Chinese with English abstract) 
Alexeter nebulator (Thunberg, 1822) 内亚力姬蜂

[New Record]

Distribution: CHINA (Jilin, Beijing).

Sheng, Sun \& Li (2020) Ichneumonids parasitizing sawflies in China (Hymenoptera, Ichneumonidae). Science Press, Beijing. (in Chinese with English abstract)

Alexeter niger (Gravenhorst, 1829) 黑亚力姬蜂

[New Record]

Distribution: CHINA (Liaoning).

Sheng, Sun \& Li (2020) Ichneumonids parasitizing sawflies in China (Hymenoptera, Ichneumonidae). Science Press, Beijing. (in Chinese with English abstract)

Campodorus ciliatus (Holmgren, 1857) 细堪姬 蜂 [New Record]

Distribution: CHINA (Liaoning).

Sheng, Sun \& Li (2020) Ichneumonids parasitizing sawflies in China (Hymenoptera, Ichneumonidae). Science Press, Beijing. (in Chinese with English abstract)

Campodorus dauricus Kasparyan, 2005 环堪姬 蜂 [New Record]

Distribution: CHINA (Liaoning).

Sheng, Sun \& Li (2020) Ichneumonids parasitizing sawflies in China (Hymenoptera, Ichneumonidae). Science Press, Beijing. (in Chinese with English abstract)

Cosmoconus caudator Kasparyan, 1971 尾角姬 蜂 [New Record]

Distribution: CHINA (Beijing, Liaoning).

Sheng, Sun \& Li (2020) Ichneumonids parasitizing sawflies in China (Hymenoptera,
Ichneumonidae). Science Press, Beijing. (in Chinese with English abstract)

Cosmoconus dlabolai Sedivy, 1971 德角姬蜂 [New Record]

Distribution: CHINA (Ningxia).

Sheng, Sun \& Li (2020) Ichneumonids parasitizing sawflies in China (Hymenoptera, Ichneumonidae). Science Press, Beijing. (in Chinese with English abstract)

Cteniscus maculiventris boreoalpinus Kerrich, 1952 上缺距姬蜂 [New Record]

Distribution: CHINA (Liaoning).

Sheng, Sun \& Li (2020) Ichneumonids parasitizing sawflies in China (Hymenoptera, Ichneumonidae). Science Press, Beijing. (in Chinese with English abstract)

Ctenochira infesta (Holmgren, 1857) 因阔鞘姬 蜂 [New Record]

Distribution: CHINA (Liaoning).

Sheng, Sun \& Li (2020) Ichneumonids parasitizing sawflies in China (Hymenoptera, Ichneumonidae). Science Press, Beijing. (in Chinese with English abstract)

Delomerista indica Gupta, 1982 印度德姬蜂 [New Record]

Distribution: CHINA (Guizhou).

Sheng, Sun \& Li (2020) Ichneumonids parasitizing sawflies in China (Hymenoptera, Ichneumonidae). Science Press, Beijing. (in Chinese with English abstract)

Delomerista mandibularis (Gravenhorst, 1829) 㓵德姬蜂 [New Record]

Distribution: CHINA (Jilin, Liaoning). 
Sheng, Sun \& Li (2020) Ichneumonids parasitizing sawflies in China (Hymenoptera, Ichneumonidae). Science Press, Beijing. (in Chinese with English abstract)

Enytus apostatus (Gravenhorst, 1829) 阿尚姬蜂

[New Record]

Distribution: CHINA (Jilin).

Sheng, Sun \& Li (2020) Ichneumonids parasitizing sawflies in China (Hymenoptera, Ichneumonidae). Science Press, Beijing. (in Chinese with English abstract)

Erromenus alpinator Aubert, 1969 山峨姬蜂 [New Record]

Distribution: CHINA (Liaoning).

Sheng, Sun \& Li (2020) Ichneumonids parasitizing sawflies in China (Hymenoptera, Ichneumonidae). Science Press, Beijing. (in Chinese with English abstract)

Euceros brevinervis Barron, 1978 短脉优姬蜂 [New Record]

Distribution: CHINA (Liaoning).

Sheng, Sun \& Li (2020) Ichneumonids parasitizing sawflies in China (Hymenoptera, Ichneumonidae). Science Press, Beijing. (in Chinese with English abstract)

Euceros clypealis Barron, 1978 唇优姬蜂 [New Record]

Distribution: CHINA (Tibet).

Sheng, Sun \& Li (2020) Ichneumonids parasitizing sawflies in China (Hymenoptera, Ichneumonidae). Science Press, Beijing. (in Chinese with English abstract)
Euceros rufocincta (Ashmead, 1906) 赤优姬蜂

[New Record]

Distribution: CHINA (Ningxia).

Sheng, Sun \& Li (2020) Ichneumonids parasitizing sawflies in China (Hymenoptera, Ichneumonidae). Science Press, Beijing. (in Chinese with English abstract)

Euceros schizophrenus Kasparya, 1984 异优姬蜂 [New Record]

Distribution: CHINA (Jilin).

Sheng, Sun \& Li (2020) Ichneumonids parasitizing sawflies in China (Hymenoptera, Ichneumonidae). Science Press, Beijing. (in Chinese with English abstract)

Euryproctus Holmgren, 1857 阔肛姬蜂属 [New Record Genus]

Distribution: CHINA (Beijing, Liaoning, Jilin, Jiangxi).

Sheng, Sun \& Li (2020) Ichneumonids parasitizing sawflies in China (Hymenoptera, Ichneumonidae). Science Press, Beijing. (in Chinese with English abstract)

Euryproctus annulatus (Gravenhorst, 1829) 环阔 肛姬蜂 [New Record]

Distribution: CHINA (Beijing, Liaoning).

Sheng, Sun \& Li (2020) Ichneumonids parasitizing sawflies in China (Hymenoptera, Ichneumonidae). Science Press, Beijing. (in Chinese with English abstract)

Euryproctus foveolatus Uchida, 1955 凹阔肛姬 蜂 [New Record]

Distribution: CHINA (Jilin). 
Sheng, Sun \& Li (2020) Ichneumonids parasitizing sawflies in China (Hymenoptera, Ichneumonidae). Science Press, Beijing. (in Chinese with English abstract)

Exenterus abruptorius (Thunberg, 1822) 锯角姬 蜂外姬蜂 [New Record]

Distribution: CHINA (Jiangxi).

Sheng, Sun \& Li (2020) Ichneumonids parasitizing sawflies in China (Hymenoptera, Ichneumonidae). Science Press, Beijing. (in Chinese with English abstract)

Exyston sibiricus (Kerrich, 1952) 西伯利亚易刻 姬蜂 [New Record]

Distribution: CHINA (Jilin).

Sheng, Sun \& Li (2020) Ichneumonids parasitizing sawflies in China (Hymenoptera, Ichneumonidae). Science Press, Beijing. (in Chinese with English abstract)

Homaspis rufina (Gravenhorst, 1829) 褐拟姬蜂

[New Record]

Distribution: CHINA (Jilin).

Sheng, Sun \& Li (2020) Ichneumonids parasitizing sawflies in China (Hymenoptera, Ichneumonidae). Science Press, Beijing. (in Chinese with English abstract)

Mesochorus niger Kusigemati, 1967 黑菱室姬蜂

[New Record]

Distribution: CHINA (Shanxi).

Sheng, Sun \& Li (2020) Ichneumonids parasitizing sawflies in China (Hymenoptera, Ichneumonidae). Science Press, Beijing. (in Chinese with English abstract)
Mesoleius aulicus (Gravenhorst, 1829) 深沟基凹 姬蜂 [New Record]

Distribution: CHINA (Jilin).

Sheng, Sun \& Li (2020) Ichneumonids parasitizing sawflies in China (Hymenoptera, Ichneumonidae). Science Press, Beijing. (in Chinese with English abstract)

Mesoleius faciator Kasparyan, 2001 颜基凹姬蜂 [New Record]

Distribution: CHINA (Beijing).

Sheng, Sun \& Li (2020) Ichneumonids parasitizing sawflies in China (Hymenoptera, Ichneumonidae). Science Press, Beijing. (in Chinese with English abstract)

Orthopelmatinae Schmiedeknecht, 1910 显唇姬 蜂亚科 [New Record Subfamily]

Distribution: CHINA (Shandong); Palaearctic and Nearctic regions.

Sun et al. (2020) Forest Pest and Disease 39(4): $1-5$.

Orthopelma Taschenberg, 1865 显唇姬蜂属 [New Record Genus]

Distribution: CHINA (Shandong); Palaearctic and Nearctic regions.

Sun et al. (2020) Forest Pest and Disease 39(4): $1-5$.

Orthopelma brevicorne Morley, 1907 短角显唇 姬蜂 [New Record]

Distribution: CHINA (Shandong).

Sun et al. (2020) Forest Pest and Disease 39(4): $1-5$. 
Otoblastus maculator Kasparyan, 1999 斑耳柄姬

蜂 [New Record]

Distribution: CHINA (Liaoning).

Sheng, Sun \& Li (2020) Ichneumonids parasitizing sawflies in China (Hymenoptera, Ichneumonidae). Science Press, Beijing. (in Chinese with English abstract)

Perilissus athaliae Uchida, 1936 枯波姬蜂 [New Record]

Distribution: CHINA (Guizhou).

Sheng, Sun \& Li (2020) Ichneumonids parasitizing sawflies in China (Hymenoptera, Ichneumonidae). Science Press, Beijing. (in Chinese with English abstract)

Phobetes nigriceps (Gravenhorst, 1829) 黑头浮 姬蜂 [New Record]

Distribution: CHINA (Liaoning).

Sheng, Sun \& Li (2020) Ichneumonids parasitizing sawflies in China (Hymenoptera, Ichneumonidae). Science Press, Beijing. (in Chinese with English abstract)

Pion japonicum Watanabe, 2016 日本针尾姬蜂 [New Record]

Distribution: CHINA (Liaoning).

Sheng, Sun \& Li (2020) Ichneumonids parasitizing sawflies in China (Hymenoptera, Ichneumonidae). Science Press, Beijing. (in Chinese with English abstract)

Pleolophus larvatus (Gravenhorst, 1829) 拉瘤角 姬蜂 [New Record]

Distribution: CHINA (Liaoning).

Sheng, Sun \& Li (2020) Ichneumonids parasitizing sawflies in China (Hymenoptera,
Ichneumonidae). Science Press, Beijing. (in Chinese with English abstract)

Pleolophus vestigialis (Förster, 1850) 短翅瘤角 姬蜂 [New Record]

Distribution: CHINA (Ningxia).

Sheng, Sun \& Li (2020) Ichneumonids parasitizing sawflies in China (Hymenoptera, Ichneumonidae). Science Press, Beijing. (in Chinese with English abstract)

Polyblastus (Polyblastus) cothurnatus (Gravenhorst, 1829) 扣多卵姬蜂 [New Record] Distribution: CHINA (Jilin).

Sheng, Sun \& Li (2020) Ichneumonids parasitizing sawflies in China (Hymenoptera, Ichneumonidae). Science Press, Beijing. (in Chinese with English abstract)

Polyblastus (Polyblastus) varitarsus fuscipes Townes, 1992 棕多卵姬蜂 [New Record]

Distribution: CHINA (Xinjiang).

Sheng, Sun \& Li (2020) Ichneumonids parasitizing sawflies in China (Hymenoptera, Ichneumonidae). Science Press, Beijing. (in Chinese with English abstract)

Polyblastus (Polyblastus) varitarsus varitarsus (Gravenhorst, 1829) 异多卵姬蜂 [New Record] Distribution: CHINA (Heilongjiang, Liaoning). Sheng, Sun \& Li (2020) Ichneumonids parasitizing sawflies in China (Hymenoptera, Ichneumonidae). Science Press, Beijing. (in Chinese with English abstract)

Protarchus testatorius (Thunberg, 1822) 褐前姬 蜂 [New Record] 
Distribution: CHINA (Jilin, Liaoning).

Sheng, Sun \& Li (2020) Ichneumonids parasitizing sawflies in China (Hymenoptera, Ichneumonidae). Science Press, Beijing. (in Chinese with English abstract)

Rhorus dauricus Kasparyan, 2012 大 壮 姬蜂 [New Record]

Distribution: CHINA (Beijing).

Sheng, Sun \& Li (2020) Ichneumonids parasitizing sawflies in China (Hymenoptera, Ichneumonidae). Science Press, Beijing. (in Chinese with English abstract)

Rhorus jinjuensis (Lee \& Cha, 1993) 简壮姬蜂 [New Record]

Distribution: CHINA (Fujian).

Sheng, Sun \& Li (2020) Ichneumonids parasitizing sawflies in China (Hymenoptera, Ichneumonidae). Science Press, Beijing. (in Chinese with English abstract)

Rhorus koreensis Kasparyan, Choi \& Lee, 2016 朝壮姬蜂 [New Record]

Distribution: CHINA (Liaoning).

Sheng, Sun \& Li (2020) Ichneumonids parasitizing sawflies in China (Hymenoptera, Ichneumonidae). Science Press, Beijing. (in Chinese with English abstract)

Rhorus lannae Reshchikov \& Xu, 2017 岗壮姬蜂 [New Record]

Distribution: CHINA (Liaoning).

Sheng, Sun \& Li (2020) Ichneumonids parasitizing sawflies in China (Hymenoptera, Ichneumonidae). Science Press, Beijing. (in Chinese with English abstract)
Rhorus nigritarsis (Hedwig, 1956) 黑跗壮姬蜂

[New Record]

Distribution: CHINA (Liaoning).

Sheng, Sun \& Li (2020) Ichneumonids parasitizing sawflies in China (Hymenoptera, Ichneumonidae). Science Press, Beijing. (in Chinese with English abstract)

Rhorus orientalis (Cameron, 1909) 东方壮姬蜂 [New Record]

Distribution: CHINA (Jiangxi).

Sheng, Sun \& Li (2020) Ichneumonids parasitizing sawflies in China (Hymenoptera, Ichneumonidae). Science Press, Beijing. (in Chinese with English abstract)

Scolobates auriculatus (Fabricius, 1804) 耳齿胫 姬蜂 [New Record]

Distribution: CHINA (Jilin, Liaoning).

Sheng, Sun \& Li (2020) Ichneumonids parasitizing sawflies in China (Hymenoptera, Ichneumonidae). Science Press, Beijing. (in Chinese with English abstract)

Scolobates nigriabdominalis Uchida, 1952 黑 齿 胫姬蜂 [New Record]

Distribution: CHINA (Liaoning).

Sheng, Sun \& Li (2020) Ichneumonids parasitizing sawflies in China (Hymenoptera, Ichneumonidae). Science Press, Beijing. (in Chinese with English abstract)

Scopesis Förster, 1869 视姬蜂属 [New Record Genus]

Distribution: CHINA (Inner Mongolia).

Sheng, Sun \& Li (2020) Ichneumonids parasitizing sawflies in China (Hymenoptera, 
Ichneumonidae). Science Press, Beijing. (in

Chinese with English abstract)

Scopesis frontator (Thunberg, 1824) 额视姬蜂

[New Record]

Distribution: CHINA (Inner Mongolia).

Sheng, Sun \& Li (2020) Ichneumonids parasitizing sawflies in China (Hymenoptera, Ichneumonidae). Science Press, Beijing. (in Chinese with English abstract)

\section{Platygastroidea 广腹细蜂总科}

\section{Scelionidae 缘腹细蜂科}

Macroteleia xui Hong \& Chen, 2020 许氏大美细

蜂 [New Species]

Holotype $q$ (SYSBM).

Host: Unknown.

Distribution: CHINA (Hebei, Yunnan, Shandong). Hong, Popovici \& Chen (2020) ZooKeys 939: 2943.

Phanuromyia ricaniae Nam, Lee \& Talamas, 2020 广翅蜡蝉亮卵蜂 [New Species]

Holotype 9 (SNU).

Host: the eggs of Ricania shantungensis Chou \& $\mathrm{Lu}$ (Hemiptera: Ricaniidae) 山东广翅蜡蝉卵.

Distribution: CHINA (Hebei, Yunnan, Shandong). Nam et al. (2020) Zootaxa 4890(1): 109-118.

Oxyscelio nullicarina Mo \& Chen, 2020 无脊尖 缘腹细蜂 [New Species]

Holotype $q$ (SCAU).

Host: Unknown.

Distribution: CHINA (Hainan, Yunnan).

Mo et al. (2020) Zootaxa 4816: 251-310.
Oxyscelio paracuculli Mo \& Chen, 2020 额拟兜 帽尖缘腹细蜂 [New Species]

Holotype $q$ (SCAU).

Host: Unknown.

Distribution: CHINA (Guangdong, Hainan, Zhejiang).

Mo et al. (2020) Zootaxa 4816: 251-310.

Gryon ancinla Kozlov \& Lê, 1996 环粒卵蜂 [New Record]

Synonym: Gryon clavaerus Kozlov \& Lê, 1996 [New Synonym]

Distribution: CHINA (Guangdong); Vietnam, Cambodia.

Chen et al. (2020) Biodiversity Data Journal 8: e47687.

Oxyscelio aclavae Burks, 2013 无棒节尖缘腹细 蜂 [New Record]

Distribution: CHINA (Hainan); Thailand, Brunei, Indonesia, Malaysia, Sri Lanka, Thaniland.

Mo et al. (2020) Zootaxa 4816: 251-310.

Oxyscelio arcus Burks, 2013 圆拱尖缘腹细蜂 [New Record]

Distribution: CHINA (Fujian); Thailand, Indonesia.

Mo et al. (2020) Zootaxa 4816: 251-310.

Oxyscelio brevidentis Burks, 2013 短齿尖缘腹细 蜂 [New Record]

Distribution: CHINA (Hainan, Yunnan); Thailand.

Mo et al. (2020) Zootaxa 4816: 251-310.

Oxyscelio excavatus (Kieffer, 1913) 无盖尖缘腹 细蜂 [New Record] 
曹焕喜, 牛泽清, 唐璞, 李涛, 朱朝东 (2021) 中国膜翅目 2020 年新分类单元. 生物多样性, 29, 1026-1034.

https://www.biodiversity-science.net/CN/10.17520/biods.2021167

Distribution: CHINA (Hainan, Zhejiang);

Philippines.

Mo et al. (2020) Zootaxa 4816: 251-310.

Oxyscelio flabelli Burks, 2013 扇尖缘腹细蜂

[New Record]

Distribution: CHINA (Yunnan); Thailand.

Mo et al. (2020) Zootaxa 4816: 251-310.

Oxyscelio jaune Burks, 2013 黄色尖缘腹细蜂

[New Record]

Distribution: CHINA (Guangxi); Thailand.

Mo et al. (2020) Zootaxa 4816: 251-310.

Oxyscelio kiefferi Dodd, 1914 基弗氏尖缘腹细

蜂 [New Record]

Distribution: CHINA (Yunnan); Philippines.

Mo et al. (2020) Zootaxa 4816: 251-310.

Oxyscelio labis Burks, 2013 唇沟尖缘腹细蜂

[New Record]

Distribution: CHINA (Yunnan); Singapore.

Mo et al. (2020) Zootaxa 4816: 251-310.

Oxyscelio mesiodentis Burks, 2013 中纵突尖缘

腹细蜂 [New Record]

Distribution: CHINA (Hainan); Malaysia, Indonesia, Laos, Nepal, Thailand.

Mo et al. (2020) Zootaxa 4816: 251-310.

Oxyscelio mollitia Burks, 2013 柔韧尖缘腹细蜂

[New Record]

Distribution: CHINA (Zhejiang, Guangdong, Yunnan); Janapn, South Korea.

Mo et al. (2020) Zootaxa 4816: 251-310.
Oxyscelio nasolabii Burks, 2013 鼻唇沟尖缘腹 细蜂 [New Record]

Distribution: CHINA (Zhejiang); Vietnam.

Mo et al. (2020) Zootaxa 4816: 251-310.

Oxyscelio nubbin Burks, 2013 小片尖缘腹细蜂 [New Record]

Distribution: CHINA (Yunnan); Thailand, Vietnam.

Mo et al. (2020) Zootaxa 4816: 251-310.

Oxyscelio ogive Burks, 2013 哥特尖拱尖缘腹细 蜂 [New Record]

Distribution: CHINA (Yunnan); Thailand, Indonesia, Vietnam.

Mo et al. (2020) Zootaxa 4816: 251-310.

Oxyscelio reflectens Burks, 2013 弯脊尖缘腹细 蜂 [New Record]

Distribution: CHINA (Yunnan); Thailand, Indonesia, Laos, Vietnam.

Mo et al. (2020) Zootaxa 4816: 251-310.

\section{Proctotrupoidea 细蜂总科}

\section{Heloridae 柄腹细蜂科}

Helorus alborzicus Izadizadeh, van Achterberg \& Talebi, 2015 厄尔布尔士山柄腹细蜂

Synonym: Helorus xinjiangensis $\mathrm{He} \& \mathrm{Xu}, 2015$ 新疆柄腹细蜂 [New Synonym]

Distribution: CHINA (Xinjiang).

Zhang et al. (2020) Zootaxa 4821(3): 570-584.

\section{Trigonalyoidea 钩腹蜂总科}

Trigonalyidae 钩腹蜂科

Jezonogonalos nyingchiensis Chen \& van Achterberg, 2020 林芝平钩腹蜂 [New Species] 
Holotype + (SYSBM).

Host: Unknown.

Distribution: CHINA (Tibet).

Chen et al. (2020) ZooKeys 918: 83-98.

Taeniogonalos eurysoma Chen \& van Achterberg, 2020 宽腹钩腹蜂 [New Species]

Holotype $q$ (SYSBM).

Host: Unknown.

Distribution: CHINA (Tibet).

Chen et al. (2020) ZooKeys 918: 83-98.

\section{Symphyta 广腰亚目}

\section{Tenthredinoidea 叶蜂总科}

\section{Argidae 三节叶蜂科}

Arge listoni Wei, 2020 高域环腹三节叶蜂 [New Species]

Holotype $q$ (ASMN).

Host plant: Unknown.

Distribution: CHINA (Gansu, Shaanxi, Henan, Shandong, Hubei, Zhejiang, Hunan, Chongqing). Chen, Wu \& Wei (2020) Entomotaxonomia 42(1): 50-56.

Arge wanziae Wei, 2020 截斑黄腹三节叶蜂

[New Species]

Holotype $q$ (ASMN).

Host plant: Unknown.

Distribution: CHINA (Zhejiang, Hunan).

Chen, Wu \& Wei (2020) Entomotaxonomia 42(1): 50-56.

\section{Cimbicidae 锤角叶蜂科}

Praia megapulvilla Yan, Li \& Wei, 2020 巨垫舌 锤角叶蜂 [New Species]

Holotype 9 (CSCS).

Host plant: Unknown.
Distribution: CHINA (Hunan).

Yan et al. (2020) Entomotaxonomia 42(3): 231239.

Praia tianmunica Yan, Li \& Wei, 2020 天目舌锤 角叶蜂 [New Species]

Holotype $q$ (CSCS).

Host plant: Unknown.

Distribution: CHINA (Zhejiang).

Yan et al. (2020) Entomotaxonomia 42(3): 231239.

Zaraea mengmeng Yan, Li \& Wei, 2020 萌萌丑 锤角叶蜂 [New Species]

Holotype 9 (LSAF).

Host plant: Padus brachypoda (Batal.) Schneid. 短梗稠李.

Distribution: CHINA (Zhejiang, Anhui).

Yan et al. (2020) Scientia Silvae Sinicae 56(5): $105-112$.

\section{Tenthredinidae 叶蜂科}

Birka jiulong Liu, Li \& Wei, 2020 九龙柄臀叶蜂

[New Species]

Holotype $q$ (ASMN).

Host plant: Unknown.

Distribution: CHINA (Zhejiang).

Liu et al. (2020) Entomotaxonomia 42(3): 240246.

Cladiucha punctata Wei, 2020 刻胸枝膜叶蜂 [New Species]

Holotype $q$ (ASMN).

Host plant: Unknown.

Distribution: CHINA (Yunnan).

Niu, Zhao \& Wei (2020) Entomotaxonomia 42(4): 311-318. 
Macrophya lishuii Li, Liu \& Wei, 2020 丽水钩瓣

叶蜂 [New Species]

Holotype $q$ (LSAF).

Host plant: Unknown.

Distribution: CHINA (Zhejiang).

Liu, Li \& Wei (2020) Alpine Entomology 4: 6572.

Macrophya nigrihistrio Li, Liu \& Wei, 2020 黑 脊钩瓣叶蜂 [New Species]

Holotype 9 (CSCS).

Host plant: Unknown.

Distribution: CHINA (Zhejiang, Hubei).

Liu, Li \& Wei (2020) Entomotaxonomia 42(1): 57-69.

Macrophya nigrotrochanterata Liu, Li \& Wei, 2020 暗转钩瓣叶蜂 [New Species]

Holotype + (LSAF).

Host plant: Unknown.

Distribution: CHINA (Liaoning).

Liu, Li \& Wei (2020) Zoologia 37: e51168.

Macrophya parahistrioides Li, Liu \& Wei, 2020

拟密纹钩瓣叶蜂 [New Species]

Holotype $q$ (CSCS).

Host plant: Unknown.

Distribution: CHINA (Taiwan).

Liu, Li \& Wei (2020) Entomotaxonomia 42(1): $57-69$.

Macrophya tianquanensis Li, Liu \& Wei, 2020 天 全钩瓣叶蜂 [New Species]

Holotype 9 (CSCS).

Host plant: Unknown.

Distribution: CHINA (Sichuan).
Liu, Li \& Wei (2020) Entomotaxonomia 42(1): $57-69$.

Pachyprotasis parawui Zhong, Li \& Wei, 2020 吴氏方颜叶蜂 [New Species]

Holotype $q$ (CSCS).

Host plant: Unknown.

Distribution: CHINA (Hunan, Zhejiang).

Zhong, Li \& Wei (2020) Entomotaxonomia 42(4): 319-328.

Pachyprotasis rufofemorata Zhong, Li \& Wei, 2020 红股方颜叶蜂 [New Species]

Holotype $q$ (CSCS).

Host plant: Unknown.

Distribution: CHINA (Hunan, Zhejiang).

Zhong, Li \& Wei (2020) Entomotaxonomia 42 (4): 319-328.

Siobla dianzangica Niu \& Wei, 2020 滇藏侧跗叶 蜂 [New Species]

Holotype $q$ (ASMN).

Host plant: Unknown.

Distribution: CHINA (Yunnan, Tibet).

Zhang, Niu \& Wei (2020) Entomotaxonomia 42(2): 130-142.

Siobla elevatina Niu \& Wei, 2020 凸缘侧跗叶蜂 [New Species]

Holotype $q$ (ASMN).

Host plant: Unknown.

Distribution: CHINA (Sichuan, Yunnan).

Tang, Niu \& Wei (2020) Entomotaxonomia 42(2): 143-162.

Siobla emeiensis Niu \& Wei, 2020 峨眉侧跗叶蜂

[New Species]

Holotype $q$ (ASMN). 
Host plant: Unknown.

Distribution: CHINA (Sichuan).

Tang, Niu \& Wei (2020) Entomotaxonomia 42(2): 143-162.

Siobla kangba Niu \& Wei, 2020 康巴侧跗叶蜂

[New Species]

Holotype $q$ (ASMN).

Host plant: Unknown.

Distribution: CHINA (Sichuan).

Tang, Niu \& Wei (2020) Entomotaxonomia 42(2): 143-162.

Siobla melanogaster Niu \& Wei, 2020 窄腹侧跗 叶蜂 [New Species]

Holotype $\widehat{\delta}$ (ASMN).

Host plant: Unknown.

Distribution: CHINA (Guizhou).

Zhang, Niu \& Wei (2020) Entomotaxonomia 42(2): 130-142.

Siobla taegeri Niu \& Wei, 2020 泰戈侧跗叶蜂

[New Species]

Holotype $q(\mathrm{ASMN})$.

Host plant: Unknown.

Distribution: CHINA (Yunnan).

Tang, Niu \& Wei (2020) Entomotaxonomia 42(2): 143-162.

Siobla trilineata Niu \& Wei, 2020 三条侧跗叶蜂

[New Species]

Holotype $q(\mathrm{ASMN})$.

Host plant: Unknown.

Distribution: CHINA (Tibet).

Zhang, Niu \& Wei (2020) Entomotaxonomia 42(2): 130-142.
Tenthredo megamaculata Xiao, Niu \& Wei, 2020

大斑长突叶蜂 [New Species]

Holotype $q$ (ASMN).

Host plant: Unknown.

Distribution: CHINA (Sichuan, Tibet).

Xiao, Niu \& Wei (2020) Zoological Systematics 45(2): 118-125.

Tenthredo nigrobullifera Xiao, Niu \& Wei, 2020 黑绿长突叶蜂 [New Species]

Holotype $q(\mathrm{ASMN})$.

Host plant: Unknown.

Distribution: CHINA (Tibet).

Xiao, Niu \& Wei (2020) Zoological Systematics 45(2): 118-125.

\section{Siricoidea 树蜂总科}

Xiphydriidae 项蜂科

Gryponeura quadrimaculata (Cameron 1899) 方 斑偏须项蜂 [New Record]

Distribution: CHINA (Shaanxi); India, Laos.

Smith (2020) Proceedings of the Entomological Society of Washington 122(1): 135-146.

\section{绝灭类群}

\section{Apocrita 细腰亚目}

\section{Ichneumonoidea 姬蜂总科}

\section{Ichneumonidae 姬蜂科}

Amplicella abbreviata Li, Shih, Kopylov \& Ren, 2020 双简巨室姬蜂 [New Species from the Lower Cretaceous Yixian Formation]

Holotype $q$ (CNUB).

Distribution: CHINA (Liaoning).

Li et al. (2020) Journal of Systematic Palaeontolog 18(11): 931-943. 


\section{Symphyta 广腰亚目}

\section{Anaxyeloidea 古锯蜂总科}

\section{Anaxyelidae 古锯蜂科}

Brachysyntexis acuta Kopylov, Rasnitsyn, Zhang

\& Zhang, 2020 畸脉短杉蜂 [New Species from the Middle Jurassic of Daohugou]

Holotype $q$ (NIGPAS).

Distribution: CHINA (Inner Mongolia).

Kopylov et al. (2020) Alcheringa: An Australasian Journal of Palaeontology 44(1): 104-114.

Brachysyntexis brevicornis Kopylov, Rasnitsyn, Zhang \& Zhang, 2020 短翅短杉蜂 [New Species from the Middle Jurassic of Daohugou]

Holotype $\hat{\sigma}$ (NIGPAS).

Distribution: CHINA (Inner Mongolia).

Kopylov et al. (2020) Alcheringa: An Australasian Journal of Palaeontology 44(1): 104-114.

Brachysyntexis laticella Kopylov, Rasnitsyn, Zhang \& Zhang, 2020 宽室短杉蜂 [New Species from the Middle Jurassic of Daohugou]

Holotype $q$ (NIGPAS).

Distribution: CHINA (Inner Mongolia).

Kopylov et al. (2020) Alcheringa: An Australasian Journal of Palaeontology 44(1): 104-114.

Brachysyntexis minuta Kopylov, Rasnitsyn, Zhang \& Zhang, 2020 小巧短杉蜂 [New Species from the Middle Jurassic of Daohugou]

Holotype $q$ (NIGPAS).

Distribution: CHINA (Inner Mongolia).
Kopylov et al. (2020) Alcheringa: An Australasian Journal of Palaeontology 44(1): 104-114.

Brachysyntexis rohwei Kopylov, Rasnitsyn, Zhang \& Zhang, 2020 罗氏短杉蜂 [New Species from the Middle Jurassic of Daohugou]

Holotype $q$ (NIGPAS).

Distribution: CHINA (Inner Mongolia).

Kopylov et al. (2020) Alcheringa: An Australasian Journal of Palaeontology 44(1): 104-114.

Brachysyntexis sinensis Kopylov, Rasnitsyn, Zhang \& Zhang, 2020 中华短杉蜂 [New Species from the Middle Jurassic of Daohugou]

Holotype $q$ (NIGPAS).

Distribution: CHINA (Inner Mongolia).

Kopylov et al. (2020) Alcheringa: An Australasian Journal of Palaeontology 44(1): 104-114.

Daosyntexis Kopylov, Rasnitsyn, Zhang \& Zhang, 2020 道虎沟杉蜂属 [New Genus from the Middle Jurassic of Daohugou]

Type species: Daosyntexis primus Kopylov, Rasnitsyn, Zhang \& Zhang, 2020; by original designation.

Distribution: CHINA (Inner Mongolia).

Kopylov et al. (2020) Alcheringa: An Australasian Journal of Palaeontology 44(1): 104-114.

Daosyntexis primus Kopylov, Rasnitsyn, Zhang \& Zhang, 2020 原始道虎沟杉蜂 [New Species from the Middle Jurassic of Daohugou] Holotype $q$ (NIGPAS).

Distribution: CHINA (Inner Mongolia). 
曹焕喜, 牛泽清, 唐㒒, 李涛, 朱朝东 (2021) 中国膜翅目 2020 年新分类单元. 生物多样性, 29, 1026-1034. https://www.biodiversity-science.net/CN/10.17520/biods.2021167

Kopylov et al. (2020) Alcheringa: An

Australasian Journal of Palaeontology 44(1):

104-114.

\section{Xyeloidea 长节叶蜂总科}

\section{Xyelidae 长节叶蜂科}

Abrotoxyela curva Zheng, Chen, Zhang \& Zhang, 2020 曲形华美长节叶蜂 [New Species from the Middle Jurassic of Daohugou]

Holotype (NIGPAS).

Distribution: CHINA (Inner Mongolia).

Zheng et al. (2020) Alcheringa: An Australasian

Journal of Palaeontology 44(1): 115-120.

Magnaxyela rara Zheng, Chen, Zhang \& Zhang, 2020 稀奇大长节叶蜂 [New Species from the Middle Jurassic of Daohugou]

Holotype $\widehat{o}$ (NIGPAS).

Distribution: CHINA (Inner Mongolia).

Zheng et al. (2020) Alcheringa: An Australasian

Journal of Palaeontology 44(1): 115-120. 


\section{References 文献来源}

Aishan Zhulidezi, Triapitsyn Serguei V. \& Hu Hong-Ying (2020) A review of the Chinese species of Lymaenon (Hymenoptera: Mymaridae), with description of six new species. Zootaxa 4834(4): 573-595. doi: 10.11646/zootaxa.4834.4.5

Astafurova Yulia V., Proshchalykin Maxim Yu, Niu Ze-Qing, Orr Michael C. \& Zhu ChaoDong (2020) New and little-known bees of the genus Sphecodes Latreille, 1804 (Hymenoptera, Apoidea, Halictidae) from Southern and South-Western China. Journal of Hymenoptera Research 79: 145-162. doi: 10.3897/jhr.79.57276

Bashir Nawaz Haider, Ma Li \& Li Qiang (2020) Chinese species of Carinostigmus Tsuneki (Hymenoptera, Crabronidae), including three new species and a new record to China. ZooKeys 987: 115-134. doi: 10.3897/zookeys.987.55317

Belokobylskij Sergey. A. \& Lin Ching-Shan (2020) A new species of the genus Ecphylus (Hymenoptera: Braconidae: Doryctinae) from Taiwan, with a diagnostic character previously unknown in the genus. Zoosystematica Rossica 29 (1): 23-32. doi: 10.31610/zsr/2020.29.1.23

Brassard François, Leong Chi-Man, Chan HoiHou \& Guénard Benoit (2020) A new subterranean species and an updated checklist of Strumigenys (Hymenoptera, Formicidae) from Macao SAR, China, with a key to species of the Greater Bay Area. ZooKeys 970: 63-116. doi: 10.3897/zookeys.970.54958

Buffington Matthew L., Giorgini Massimo, Lue Chia-Hua, Formisano Giorgio, Cascone
Pasquale, Forshage Mattias, Driskell Amy \& Guerrieri Emilio (2020) Description of the aberrant Leptopilina lasallei n. sp., with an updated phylogeny of Leptopilina Förster (Hymenoptera: Figitidae: Eucoilinae). Journal of Natural History 54(9-12): 565583. doi: 10.1080/00222933.2020.1754483

Cao Liang-Ming, van Achterberg Cornelis, Tang Yan-Long, Wang Xiao-Yi \& Yang ZhongQi (2020) Revision of parasitoids parasitizing Massicus raddei (Blessig \& Solsky) (Coleoptera, Cerambycidae) in China, with one new species and genus. Zootaxa 4881 (1): 104-130. doi: 10.11646/zootaxa.4881.1.7

Chatthanabun Nontawat, Ascher John S., Pinkaew Nantasak, Thanoosing Chawatat, Traiyasut Prapun \& Warrit Natapot (2020) Resin bees of genus Megachile, subgenera Callomegachile and Carinula (Hymenoptera, Megachilidae) from Thailand with description of a new species. ZooKeys 997: 95-114. doi: 10.3897/zookeys.997.34935

Chen Hua-Yan, Hong Chun-Dan, van Achterberg Cornelis \& Pang Hong (2020) New species and new records of Trigonalyidae (Hymenoptera) from Tibet, China. ZooKeys 918: 83-98. doi: 10.3897/zookeys.918.49729

Chen Hua-Yan \& Azevedo Celso O. (2020) Discovery of a couple of Foenobethylus (Hymenoptera, Bethylidae) in copulation suggests a synonym under Parascleroderma. Journal of Asia-Pacific Entomology 23(4): 1241-1247. doi:10.1016/j.aspen.2020.10.001

Chen Hua-Yan, Olmi Massimo, Pang Hong \& Liu Jing-Xian (2020) Application of DNA 
barcoding confirms the host of Gonatopus viet Olmi, 1986 (Hymenoptera, Dryinidae). ZooKeys 944: 115-120. doi: 10.3897/zookeys.944.53054

Chen Huayan, Talamas Elijah J, Bon MarieClaude \& Moore Matthew R. (2020) Gryon ancinla Kozlov \& Lê (Hymenoptera: Scelionidae): host association, expanded distribution, redescription and a new synonymy. Biodiversity Data Journal 8: e47687. doi: doi.org/10.3897/BDJ.8.e47687

Chen Yaping, Wu Ruoxuan \& Wei Meicai (2020) Two new species of Arge Schrank (Hymenoptera: Argidae) from China. Entomotaxonomia 42(1): 50-56. doi: 10.11680/entomotax.2020007

Danilov Yuriy N. \& Byvaltsev Alexander M. (2020) The digger wasps of the genus Palmodes Kohl, 1890 in Central Asia (Hymenoptera: Sphecidae: Prionychini). Zootaxa 4803 (3): 401-434. doi: 10.11646/zootaxa.4803.3.1

Davidian E.M. (2020) New species of the genus Praon Haliday, 1833 (Hymenoptera: Aphidiidae) from China. Caucasian Entomological Bulletin 16 (2): 207-211. doi: 10.23885/181433262020162-207211 (in Russian with English abstract)

Duan Chun-Hua, Li Tao, Wu Hai-Wei \& Sheng Mao-Ling (2020) A new species of genus Endasys Förster (Ichneumonidae, Cryptinae) parasitizing Pristiphora (Tenthredinidae) and a key to species from China. Zootaxa 4743(1): 111-118. doi: 10.11646/zootaxa.4743.1.9

Fang Zhiqiang, Tang Chang-Ti., Nicholls J. A., Zhu Ying, Xiong Tieyi, Hearn Jack, Sinclair Frazer, Melika George, Nieves-Aldrey Jose
Luis, Csoka Gyorgy, Mikolajczak Katarzyna M, Stone Graham N \& Fang Shengguo (2020) A New Genus of Oak Gallwasp, Heocynips Fang, Nieves-Aldrey, and Melika (Hymenoptera: Cynipidae: Cynipini), from China. Proceedings of the Entomological Society of Washington 122(4): 787-804. doi: doi.org/10.4289/0013-8797.122.4.787

Fang Zhiqiang, Tang Chang-Ti, Zhu Ying, Xiong Tieyi, Sinclair Frazer, Hearn Jack, Mikolajczak Katarzyna M, Melika George Melika, Stone Graham N, Fang Shengguo (2020) Lithosaphonecrus edurus Fang, Melika, and Tang, a New Cynipid Inquiline Species (Hymenoptera: Cynipidae: Synergini) from Sichuan, China. Proceedings of the Entomological Society of Washington 122(4): 805-820. doi: 10.4289/0013-8797.122.4.805

Gates Michael W., Chao Yi-Min, Lin Sheng-Feng \& Yang Man-Miao (2020) Description of two new Quadrastichus (Hymenoptera: Eulophidae) reared from Litchiomyia chinensis (Diptera: Cecidomyiidae) on commercial lychee (Litchi chinensis; Sapindaceae) in Taiwan. Journal of Natural History 54 (9-12): 635-646. doi:10.1080/00222933.2020.1779367

Ho Yu-Hsiang, Hsiao Yun, Terayama Mamoru \& Chan Mei-Ling (2020) Ultramorphological Characteristics of Falsogastrallus sauteri Pic (Coleoptera: Ptinidae) and a New Species of Cephalonomia Westwood (Hymenoptera: Bethylidae): A Book-Boring Beetle and Its Natural Enemy in Taiwan. $\begin{array}{llll}\text { Insects } & 11 & \text { (4): } 223 . & \text { doi: }\end{array}$ 10.3390/insects11040223 
Hong Chun-Dan, Popovici Ovidiu Alin \& Chen Hua-Yan (2020) Notes on Macroteleia Westwood (Hymenoptera, Scelionidae) from China, with description of a new species. ZooKeys 939: 29-43. doi: 10.3897/zookeys.939.51272

Humala Andrei E., Lee Jong-Wook \& Choi JinKyung (2020) A review of the genus Orthocentrus Gravenhorst (Hymenoptera, Ichneumonidae, Orthocentrinae) from South Korea. Journal of Hymenoptera Research 75: 15-65. doi: 10.3897/jhr.75.47006

Jin Xiang-Xiang \& Li Cheng-De (2020) A new species of Ooctonus from China, with a revised key to the Chinese species (Hymenoptera: Mymaridae). Oriental Insects 54(3): 319-326. doi: 10.1080/00305316.2019.1625825

Kocić Korana, Petrović Andjeljko, Čkrkić Jelisaveta, Kavallieratos Nickolas G., Rakhshani Ehsan, Arnó Judit, Aporicio Yahana, Hebert Paul D. N. \& Tomanović Željko (2020) Resolving the taxonomic status of potential biocontrol agents belonging to the neglected genus Lipolexis Förster (Hymenoptera, Braconidae, Aphidiinae) with descriptions of six new species. Insects 11(10): 667. doi:10.3390/insects 11100667

Kopylov Dmitry S., Rasnitsyn Alexander P., Zhang Haichun \& Zhang Qi (2020) Anaxyelidae of Daohugou: oldest occurrencesof the relict family in the fossil record. Part 1: Daosyntexis and Brachysyntexis. Alcheringa: An Australasian Journal of Palaeontology 44(1): 104-114. doi:10.1080/03115518.2019.1697753
Kostro-Ambroziak Agata, Zhou Zichen \& Reshchikov Alexey (2020) A new species of Phytodietus $\quad 1829$ (Hymenoptera: Ichneumonidae: Tryphoninae) from China. Zootaxa 4877(2): 391-395. doi: 10.11646/zootaxa.4877.2.11

Le Ngoc Hoan, Nahrung Helen F., Morgan Jess A.T. \& Lawson Simon A. (2020) Multivariate ratio analysis and DNA markers reveal a new Australian species and three synonymies in eucalypt-gall-associated Megastigmus

(Hymenoptera: Megastigmidae). Bulletin of Entomological Research 110 (6): 709-724. doi: 10.1017/S000748532000022X

Lelej A. S. (2020) Three new species of velvet ants from the tribe Smicromyrmini (Hymenoptera: Mutillidae) with unusual color syndrome. Far Eastern Entomologist 421: 1-9. doi: 10.25221/fee.421.1

Lelej A. S. (2020) To the knowledge of velvet ants of the genera Artiotilla Invrea, Radoszkowskitilla Lelej and Taiwanomyrme Tsuneki (Hymenoptera: Mutillidae). Far Eastern Entomologist 410: 1-10. doi: 10.25221/fee. 410.1

Li Longfeng, Shih Peter J. M., Kopylovd Dmitry S., Li Daqing \& Ren Dong (2020) Geometric morphometric analysis of Ichneumonidae (Hymenoptera: Apocrita) with two new Mesozoic taxa from Myanmar and China. Journal of Systematic Palaeontolog 18(11): 931-943.

doi:10.1080/14772019.2019.1697903

Li Jun, Zheng Min-Lin \& Chen Jia-Hua (2020) First record of Rilipertus Haeselbarth (Hymenoptera: Braconidae: Euphorinae) from China with description of a new species. 
Zootaxa $4851 \quad$ (1): 198-200. doi:

10.11646/zootaxa.4851.1.11

Li Jun, van Achterberg Cornelis, Zheng Min-Lin \& Chen Jia-Hua (2020) A new species of Myiocephalus Marshall (Hymenoptera, Braconidae, Euphorinae) from China. ZooKeys 933: 95-105. doi: 10.3897/zookeys.933.49607

Li Jun, van Achterberg Cornelis, Zheng Minlin \& Chen Jiahua (2020) Review of Neoneurini Bengtsson (Hymenoptera: Braconidae: Euphorinae) from China. Zoological Systematics 45(4): 281-289. doi: 10.11865/zs.202034

Li Tao, Sun Shu-Ping \& Sheng Mao-Ling (2020) A new species of genus Rhinotorus Förster (Ichneumonidae, Ctenopelmatinae) parasitizing Pristiphora erichsonii (Hymenoptera, Tenthredinidae) and a key to Eastern Palaearctic species. Journal of Hymenoptera Research 77: 203-211. doi: 10.3897/jhr.77.53323

Li Tao, Sun Shu-Ping \& Sheng Mao-Ling (2020) First record of the genus Microstenus Szépligeti (Hymenoptera: Ichneumonidae: Cryptinae) from China, with description of one new species and a key to world species. Zootaxa 4838: 594-600. doi: 10.11646/zootaxa.4838.4.10

Li Wen-Jian \& Li Cheng-De (2020) A new species of Oomyzus Rondani (Hymenoptera, Eulophidae) and first record of $O$. gallerucae (Fonscolombe) from China, with a key to Chinese species. ZooKeys 950: 41-49. doi: 10.3897/zookeys.950.48795

Li Xiao-Li \& Ma Li (2020) Two New Record Species and Genus of Crabronidae (Hymenoptera) from China. Southwest
China Journal of Agricultural Sciences 33

(6): 1332-1336. doi: 10 . 16213

/j. cnki. scjas. 2020. 6. 036 (in Chinese with English abstract) [李晓莉, 马 丽 (2020) 中国方头泥蜂科一新纪录属及 二新记录种记述 (膜翅目: 方头泥蜂科). 西 南农业学报 33(6): 1332-1336.]

Li Yang, Tang Pu \& Chen Xue-Xin (2020) The genus Indabracon van Achterberg (Hymenoptera, Braconidae, Braconinae) in China, with description of four new species. Journal of Hymenoptera Research 76: 3956. doi: doi.org/10.3897/jhr.76.50794

Li Yang, He Jun-Hua \& Chen Xue-Xin (2020) Review of six genera of Braconinae Nees (Hymenoptera, Braconidae) in China, with the description of eleven new species. Zootaxa 4818(1): 1-74. doi: 10.11646/zootaxa.4818.1.1

Li Yang, He Jun-Hua \& Chen Xue-Xin (2020) The subgenera Glabrobracon Fahringer, Lucobracon Fahringer and Uncobracon Papp of the genus Bracon Fabricius (Hymenoptera, Braconidae, Braconinae) in China, with the description of eleven new species. Deutsche Entomologische Zeitschrift 67: 209-252. doi: 10.3897/dez.67.57668

Li Yang, van Achterberg Cornelis \& Chen XueXin (2020) Two genera Campyloneurus Szépligeti and Iphiaulax Foerster in China, with the descriptions of fourteen new species (Hymenoptera, Braconidae, Braconinae). Zootaxa 4884(1): 1-67. doi: doi.org/10.11646/zootaxa.4884.1.1

Li Zhuomiao, Yao Tingting, Xu Zhihong, Meng Ling \& Li Baoping (2020) A new species of Cheiloneurus Westwood (Hymenoptera, 
Encyrtidae) as a hyperparasitoid of the invasive cotton mealybug, Phenacoccus solenopsis Tinsley, in China. ZooKeys 974: 23-29. doi: 10.3897/zookeys.974.55528

Liu Mengmeng, Li Zejian \& Wei Meicai (2020) Key to the Macrophya sibirica group (Hymenoptera: Tenthredinidae) with description of a new species from China. Zoologia 37: e51168. doi: 10.3897/zoologia.37.e51168

Liu Mengmeng, Li Zejian \& Wei Meicai (2020) Key to the Macrophya zhaoae group (Hymenoptera, Tenthredinidae) with description of a new species from China. Alpine Entomology 4: 65-72. doi: 10.3897/alpento.4.48232

Liu Mengmeng, Li Zejian \& Wei Meicai (2020) Three new species of the Macrophya histrio group (Hymenoptera: Tenthredinidae) with a key to species from China. Entomotaxonomia 42 (1): 57-69. doi: 10.11680/entomotax.2020008

Liu Mengmeng, Li Xiufang, Yang Xiaojun, Li Zejian \& Wei Meicai (2020) A new species of Birka Malasise (Hymenoptera: Tenthredinidae) from Lishui, China. Entomotaxonomia 42 (3): 240-246. doi: 10.11680/entomotax.2020021

Liu Zhen, He Jun-Hua, Chen Xue-Xin \& Gupta Ankita (2020) The ater-group of the genus Apanteles Foerster (Hymenoptera, Braconidae, Microgastrinae) from China with the descriptions of forty-eight new species. Zootaxa 4807(1): 1-205. doi: 10.11646/zootaxa.4807.1.1

Lu Ciding, Tang Jinhan, Dong Wangying, Zhou Youjun, Gai Xinmin, Lin Haoyu, Song Dongbao \& Liang Guanghong (2020) A new species of Glyptapanteles Ashmead (Hymenoptera, Braconidae, Microgastrinae) within Macrobrochis gigas (Lepidoptera, Arctiidae, Lithosiidae) in Fujian, China. ZooKeys 913: 127-139. doi: 10.3897/zookeys.913.46646

Luo Li, Zhang Qiao-Hua, Chen Bin \& Li TingJing (2020) Additions to the knowledge of the genus Allorhynchium van der Vecht, 1963 from China (Hymenoptera, Vespidae, Eumeninae). Journal of Hymenoptera Research 77: 119-137. doi: doi.org/10.3897/jhr.77.52309

Ma Li \& Li Qiang (2020) The genus Polemistus de Saussure in China with descriptions of four new species (Hymenoptera: Crabronidae). Zootaxa 4852(4): 475-484. doi: 10.11646/zootaxa.4852.4.5

Mo Wen-Hui, Chen Hua-Yan, Johnson Norman F., Pang Hong, Ma Li \& Liu Jing-Xian (2020) Revision of the genus Oxyscelio kieffer (Hymenoptera, Scelionidae) from China. Zootaxa 4816: 251-310. doi: 10.11646/zootaxa.4816.3.1

Nam Sanghyeok, Chen Hua-Yan, Talamas Elijah J., Lee Gwan-Seok, Dong Wei, Sun Li-Juan \& Lee Seunghwan (2020) Phanuromyia ricaniae Nam, Lee \& Talamas sp. n. (Hymenoptera: Scelionidae) reared from the eggs of Ricania shantungensis Chou \& Lu (Hemiptera: Ricaniidae) in Asia. Zootaxa $4890 \quad$ (1): 109-118. doi: 10.11646/zootaxa.4890.1.6

Niu Gengyun, Zhang Hang, Wei Meicai (2020) Review of Cladiucha Konow (Hymenoptera: Tenthredinidae) with description of a new species from China. Entomotaxonomia 
42(4): $\quad$ 311-318. doi:

10.11680/entomotax.2020031

Niu Ze-Qing, Yuan Feng, Ascher John S., Kasparer Max, Orr Michael C., Griswold Terry, Zhu Chao-Dong (2020) Bees of the genus Anthidium Fabricius, 1804 (Hymenoptera: Apoidea: Megachilidae: Anthidiini) from China. Zootaxa 4867(1): 001-067. doi: 10.11646/zootaxa.4867.1.1.

Niu Ze-Qing, Zhang Dan \& Zhu Chao-Dong (2020) Extraordinary bees of the genus Lasioglossum Curtis, 1833 (Hymenoptera: Apoidea: Halictidae) from China. Zoological Systematics 45(1): 50-58. doi: 10.11865/zs.202005

Okayasu Juriya (2020) Velvet ants of the tribe Smicromyrmini Bischoff (Hymenoptera: Mutillidae) of Japan. Zootaxa 4723(1): 001110. doi: 10.11646/zootaxa.4723.1.1

Okido Hirofumi, Ogata Kazuo \& Hosoishi Shingo (2020) Taxonomic revision of the ant genus Myrmecina in Southeast Asia (Hymenoptera: Formicidae). Bulletin of the Kyushu University Museum (17): 1-108. doi: $10.15017 / 3077267$

Pang Yin, Liu Zhiwei, Su Cheng-Yuan \& Zhu Dao-Hong (2020) A new species of Periclistus Foerster, 1869 from China and review of the tribe Diastrophini (Hymenoptera, Cynipoidea, Cynipidae). ZooKeys 964: 109-126. doi: 10.3897/zookeys.964.47441

Peng Lingfei, Gibson Gary A. P., Tang Lu \& Xiang Jiawei (2020) Review of the species of Anastatus (Hymenoptera: Eupelmidae) known from China, with description of two new species with brachypterous females.
Zootaxa 4767(3): 351-401. doi: 10.11646/zootaxa.4767.3.1

Pham Nhi Thi, Matsumoto Rikio, Konishi Kazuhiko, Sheng Mao-Ling \& Broad Gavin R. (2020) A review of the genus Apophysius Cushman (Ichneumonidae: Phygadeuontinae) with descriptions of six new species. Zootaxa 4802(2): 301-316. doi: 10.11646/zootaxa.4802.2.5

Pujade-Villar Juli, Wang Yiping \& Lobato-Vila Irene (2020) Lithosaphonecrus puigdemonti sp. nov. from China (Hymenoptera: Cynipidae). Entomotaxonomia 42(1): 7080. doi: 10.11680/entomotax.2020009

Pujade-Villar Juli, Wang Yiping, Cuesta-Porta Victor, Guo Rui, Nicholls James A. \& Melika George (2020) Andricus forni Pujade-Villar \& Nicholls n. sp., a new species of oak gallwasp from China (Hymenoptera: Cynipidae). Zootaxa 4890(4): $\quad$ 554-566. doi:

10.11646/zootaxa.4890.4.7

Pujade-Villar Juli, Wang Yiping, Guo Rui, SalaNishikawa Alba, Cuesta-Porta Victor, Arnedo Miquel A. \& Melika George (2020) A new species of Cerroneuroterus Melika Pujade-Villar from the Eastern Palaearctic (Hymenoptera, Cynipidae, Cynipini). Zootaxa 4869(4): 515-528. doi: 10.11646/zootaxa.4869.4.3

Pujade-Villar Juli, Wang Yiping, Zhang Wenli, Mata-Casanova Noel, Lobato-Vila Irene, Dénes Avar-Lehel \& László Zoltán (2020) A new Diplolepis Geoffroy (Hymenoptera, Cynipidae, Diplolepidini) species from China: a rare example of a rose gall-inducer of economic significance. ZooKeys 904: 131-146. doi: 10.3897/zookeys.904.46547 
Ranjith A.P., van Achterberg Cornelis, Sankararaman H. \& Nasser M. (2020) Discovery of the ichneutine genus Paroligoneurus Muesebeck (Hymenoptera: Braconidae) from the Indian subcontinent with the description of a new species from Northwest India. Zootaxa 4786 (3): 396-408. doi: 10.11646/zootaxa.4786.3.5

Seifert Bernhard (2020) A taxonomic revision of the Palaearctic members of the subgenus Lasius s. str. (Hymenoptera, Formicidae). Soil Organisms 92(1): 15-86. doi: 10.25674/so92iss 1 pp 15

Sheng Mao-Ling, Sun Shu-Ping \& Li Tao (2020) Ichneumonids parasitizing sawflies in China (Hymenoptera, Ichneumonidae). Science Press, Beijing. (in Chinese with English abstract) [盛茂领, 孙淑萍, 李涛 (2020) 寄 生叶蜂的姬蜂科天敌昆虫. 科学出版社, 北京.]

Si Yu, Huang Jian, Wang Zhu-Hong (2020) A newly recorded species, Aphytis sankarani Rosen et DeBach (Hymenoptera: Aphelinidae) from China and the ontogeny of its pupa. Journal of Environmental Entomology 42(5): 1216-1222. doi: 10.3969/j.issn.1674-0858.2020.05.22 （in Chinese with English abstract) [司宇, 黄建, 王竹红 (2020) 中国黄蚜小蜂属一新记录 种及其蛹的个体发育. 环境昆虫学报 42(5): 1216-1222.]

Smith David R. (2020) New Species and New Records of Xiphydriid Woodwasps (Hymenoptera: Xiphydriidae) from Southeastern Asia. Proceedings of the Entomological Society of Washington 122(1): 135-146. doi: 10.4289/00138797.122.1.135
Song Hai-Tian, Fei Ming-Hui, Li Bao-Ping, Zhu Chao-Dong \& Cao Huan-Xi (2020) A new species of Oomyzus Rondani (Hymenoptera, Eulophidae) reared from the pupae of Coccinella septempunctata (Coleoptera, Coccinellidae) in China. ZooKeys 953: 4960. doi: 10.3897/zookeys.953.53175

Sun Shu-Ping, Li Tao, Sheng Mao-Ling \& Lü Jun (2020) The species of Campodorus Förster, 1869 and a related species (Hymenoptera, Ichneumonidae) from China. European Journal of Taxonomy (658). doi: 10.5852/ejt.2020.658

Sun Shu-Ping, Wang Tao, Li Tao \& Sheng MaoLing (2020) Two new species of Strongylopsis Brauns (Hymenoptera, Ichneumonidae, Pimplinae) from Beijing, and a key to all species. Journal of Hymenoptera Research 79: 1-13. doi: 10.3897/jhr.79.57726

Sun Shu-Ping, Zhao Tao, Sun Yu-Jiang, Li Tao, Pan Tao (2020) New Chinese record of Orthopelmatinae Taschenberg (Hymenoptera: Ichneumonidae) parasitizing cynipid. Forest Pest and Disease 39(4): 1-5. doi: 10. 19688 /j. cnki. issn1671 0886. 20200015 (in Chinese with English abstract) [孙淑萍, 赵涛, 孙玉江, 李涛, 潘涛 (2020) 中国发现寄生瘞蜂的显唇姬蜂. 中 国森林病虫 39(4): 1-5.]

Tang Chang-Ti, Melika George, Nicholis James A., Yang Man-Miao \& Stone Graham N. (2020) A New Cycloneuroterus Melika \& Tang Oak Gallwasp Species (Hymenoptera: Cynipidae: Cynipini) Associated with Lithocarpus (Fagaceae) from Taiwan. Proceedings of the Entomological Society 
of Washington 122(1): 184-196. doi:

$10.4289 / 0013-8797.122 .1 .184$

Tang Xingyu, Niu Gengyun, Wei Meicai (2020)

Four new species and a key to species of the

Siobla sturmii group (Hymenoptera:

Tenthredinidae). Entomotaxonomia 42(2):

143-162. doi: 10.11680/entomotax.2020015

Taylor Christopher K., Barthélémy Christophe,

Roy Cheung Shun Chi \& Guénard Benoit (2020) Review of Psenulus species (Hymenoptera, Psenidae) in the Hong Kong SAR, with description of three new species.

Journal of Hymenoptera Research 79: 169-

211. doi: $10.3897 /$ jhr.79.55832

Tian Xiao-Xia, van Achterberg Cornelis, Wu Jia-

Xuan \& Tan Jiang-Li (2020) New

Gnamptodontinae

(Hymenoptera:

Braconidae) from China and Vietnam, with two genera new for China and seven new species. Zootaxa 4778 (3): 471-508. doi: 10.11646/zootaxa.4778.3.3

Triapitsyn Serguei Vladimirovich, Shih HsienTzung \& Huang Shou-Horng (2020) Identification of egg parasitoids of rice leafhoppers and planthoppers (Hemiptera: Cicadellidae and Delphacidae) of economic importance in Taiwan, part 2: Trichogrammatidae (Hymenoptera).

Journal of Asia-Pacific Entomology 23(4): 970-980. doi: 10.1016/j.aspen.2020.08.004

Vas Zoltán (2020) New species and records of Afrotropical, Australasian, Oriental and Palaearctic Casinaria Holmgren, 1859 (Hymenoptera: Ichneumonidae: Campopleginae). Opuscula Zoologica Budapest 51(2): 133-152. doi: 10.18348/opzool.2020.2.133
Vas Zoltán (2020) New species and records of Charops Holmgren, 1859 (Hymenoptera: Ichneumonidae: Campopleginae). Acta Zoologica Academiae Scientiarum Hungaricae 66(3): 247-267. doi: 10.17109/AZH.66.3.247.2020

Wang Hua-Chuan, Chen Bin \& Li Ting-Jing (2020) Taxonomy of the genus Ectopioglossa Perkins from China, with two new species and an updated key to the Oriental species (Hymenoptera: Vespidae: Eumeninae). Journal of Asia-Pacific Entomology 23(2): 253-259. doi: 10.1016/j.aspen.2019.11.008

Wang Zhu Hong, Xu Li Yue, Si Yu, Huang Jian, Schmidt Stefan, Polaszek Andrew \& Geng Hui (2020) The species of the varius group of Coccophagus (Hymenoptera: Aphelinidae) from China, with description of a new species, DNA sequence data, and a new country record. Journal of Natural History 54(29-30): 1879-1896. doi: 10.1080/00222933.2020.1831093

Wei Ya-Wei, Zhou Yong-Bin, Zou Qing-Chi \& Sheng Mao-Ling (2020) A new species of Campoletis Förster (Hymenoptera, Ichneumonidae) with a key to species known from China, Japan and South Korea. ZooKeys 1004: 99-108. doi: 10.3897/zookeys.1004.57913

Wong Tsz Long \& Guénard Benoit (2020) Review of ants from the genus Polyrhachis Smith (Hymenoptera: Formicidae: Formicinae) in Hong Kong and Macau, with notes on their natural history. Asian Myrmecology 13: e013001

Xiao Hui, Yan Jiahe (2020) A new genus of Pteromalidae (Hymenoptera) with two new 
曹焕喜, 牛泽清, 唐㒒, 李涛, 朱朝东 (2021) 中国膜翅目 2020 年新分类单元. 生物多样性, 29, 1026-1034. https://www.biodiversity-science.net/CN/10.17520/biods.2021167

species, parasitoids of larvae of Stenocephus fraxini Wei (Hymenoptera: Cephidae) from China. Zoological Systematics 45(3): 231235. doi: 10.11865/zs.202026

Xiao Wei, Niu Gengyun \& Wei Meicai (2020) Two new species of the Tenthredo grahami group from China (Hymenoptera: Tenthredinidae), with a revised key to species. Zoological Systematics 45(2): 118125. doi: $10.11865 /$ zs.202016

Yan Cheng-Jin, Wu Qiong, He Jun-Hua \& Chen Xue-Xin (2020) The genus Aspicolpus Wesmael, 1838 (Hymenoptera, Braconidae, Brachistinae) from China, with descriptions of four new species. Zootaxa 4743(3): 391401. doi: 10.11646/zootaxa.4743.3.6

Yan Yuchen, Chen Ling, Li Guihong, Li Zejian \& Wei Meicai (2020) Two new species of the Praia Wankowicz (Hymenoptera: Cimbicidae) from China. Entomotaxonomia 42(3): 231-239. doi: 10.11680/entomotax.2020020

Yan Yuchen, Li Zejian, Li Guihong \& Wei Meicai (2020) A new species of Zaraea (Leach, 1817) (Hymenoptera, Cimbicidae) from China Harming Padus brachypoda. Scientia Silvae Sinicae 56(5): 105-112. doi: 10.11707/j.1001-7488.20200512 (in Chinese with English abstract) [晏毓晨, 李 泽建, 黎桂鸿, 魏美才 (2020) 危害短梗稠 李的中国丑锤角叶蜂属(膜翅目: 锤角叶 蜂科)一新种. 林业科学 56 (5): 105-112.]

Yang Xiao-Hui, Liu Zhiwei, Pang Yin, Su ChengYuan \& Zhu Dao-Hong (2020) Three new species of Synergini wasps from same galls on Lithocarpus glaber (Thunb.) in Hunan, China (Hymenoptera, Cynipidae). Insect
Systematics \& Evolution 51: 551-583. doi: 10.1163/1876312X-00002304

Yang Zhong-Qi, Wang Xiao-Yi, Zhong Xin, Liu Xin, Cao Liang-Ming \& Wang Hai-Zhen (2020) A new species of Pteromalus (Hymenoptera: Pteromalidae) parasitizing pupa of Gynaephora. Scientia Silvae Sinicae 56(2): $\quad 99-105 . \quad$ DOI: 10.11707/j.1001-7488.20200211 (in Chinese with English abstract) [杨忠岐, 王 小艺, 钟欣, 刘昕, 曹亮明, 王海贞 (2020) 寄生青海草原毛虫的金小蜂一新种 (膜翅 目: 金小蜂科). 林业科学 56(2): 99-105. ]

Zhang Dan, Niu Ze-Qing, Orr Michael C., Ascher John S. \& Zhu Chao-Dong (2020) Chinese species of Nomia (Gnathonomia) Pauly, 2005 (Hymenoptera: Apoidea: Halictidae: Nomiinae). Zootaxa 4768(1): 076-094. doi: 10.11646/zootaxa.4768.1.5

Zhang Ruo-Nan, van Achterberg Cornelis, Tian Xiao-Xia, He Cheng-Yi \& Tan Jiang-Li (2020) Sexual variation in two species of Helorus Latreille (Hymenoptera, Heloridae) from NW China, with description of female of Helorus caii He \& Xu. Zootaxa 4821(3): 570-584. doi: 10.11646/zootaxa.4821.3.8

Zhang Ruo-Nan, van Achterberg Cornelis, Tian Xiao-Xia \& Tan Jiang-Li (2020) Review of the Bobekia-group (Braconidae, Alysiinae, Alysiini), with description of a new genus and a new subgenus. ZooKeys 926: 25-51. doi: 10.3897/zookeys.926.47270

Zhang Xue, Chen Bin \& Li Ting-Jing (2020) A taxonomic revision of Allodynerus Blüthgen (Hymenoptera: Vespidae: Eumeninae) from China. Zootaxa 4750(4): 545-559. doi: 10.11646/zootaxa.4750.4.5 
Zhang Xue, Chen Bin \& Li Ting-Jing (2020) Taxonomy of the genus Epsilon from China, with a new species and an updated key to the Oriental species (Hymenoptera, Vespidae, Eumeninae). Zookeys 910: 131-142. doi: 10.3897/zookeys.910.35846

Zhang Yiwen, Niu Gengyun, Wei Meicai (2020) Three new species and a key to species of the Siobla grahami group (Hymenoptera: Tenthredinidae) from China.

Entomotaxonomia 42(2): 130-142. doi: 10.11680/entomotax.2020011

Zheng Minlin \& Song Dongbao (2020) A review of Deuterixys Mason from China, with description of three new species (Hymenoptera, Braconidae, Microgastrinae). Zootaxa 4728(4): 489-497. doi: 10.11646/zootaxa.4728.4.8

Zheng Yan, Chen Jun, Zhang Junqiang \& Zhang Haichun (2020) New fossil sawflies (Hymenoptera, Xyelidae) from the Middle Jurassic of northeastern China. Alcheringa: An Australasian Journal of Palaeontology 44(1): 115-120. doi: 10.1080/03115518.2019.1641618

Zhong Yihai, Li Zejian \& Wei Meicai (2020) A key to species of the Pachyprotasis flavipes group (Hymenoptera: Tenthredinidae) with two new species from China. Entomotaxonomia 42 (4): 319-328. doi: 10.11680/entomotax.2020033

Zhou SY, Chen DN \& Chen ZL (2020) Discovery of novel Ooceraea (Hymenoptera: Formicidae: Dorylinae) species with 8segmented antennae from China. Sociobiology 67(2): 139-143. doi: 10.13102/sociobiology.v67i2.3740
$\mathrm{Zu}$ Guo-Hao, Yang Ze-Ning, Cao Xin-Sheng, Wang Yuan-Hong \& Li Cheng-De (2020) Two new species of Encyrtidae (Hymenoptera: Chalcidoidea) from China, parasitoid of Antecerococcus roseus (Green) (Hemiptera: Cerococcidae) on Boehmeria nivea (L.) Gaud. Phytoparasitica 48: 43-50. doi: 10.1007/s12600-019-00775-6

$\mathrm{Zu}$ Guohao, Zhang Chen, Yang Zening \& Li Chengde (2020) A new species of Exoristobia (Hymenoptera: Encyrtidae) from China. Journal of Forestry Research. doi: 10.1007/s11676-020-01218-3

Zu Guo-Hao, Yang Ze-Ning \& Li Cheng-De (2020) One new species and one new record species of Rhopus Förster (Hymenoptera: Encyrtidae) from China. Journal of Northeast Forestry University 48 (1): 115117. doi: 10.13759/j.cnki.dlxb.2020.01.021 (in Chinese) [祖国浩, 杨泽宁, 李成德 (2020) 裂脖跳小蜂属 (膜翅目: 跳小蜂科) 1 新种 及 1 新记录记述. 东北林业大学学报 48 (1): 115-117.]

$\mathrm{Zu}$ Guo-Hao, Yang Ze-Ning, Chen Ye \& Li Cheng-De (2020) One new species of Cerapteroceroides (Hymenoptera: Encyrtidae). Journal of Northeast Forestry University 48 (1): 118-119. doi: 10.13759/j.cnki.dlxb.2020.01.022 (in Chinese with English abstract) [祖国浩, 杨 泽宁, 陈业, 李成德 (2020) 纹翅跳小蜂属 (膜翅目: 跳小蜂科) 1 新种. 东北林业大学 学报 48(1): 118-119. 


\section{Abbreviations of holotypes' depositories}

正模标本保藏地缩写列表

\begin{tabular}{|c|c|c|}
\hline 缩写 … & 英文全称 & 中文全称 \\
\hline Abbreviation & Full Form in English & Full Form in Chinese \\
\hline ASMN & Asian Sawfly Museum, Nanchang, CHINA & 南昌亚洲叶蜂标本馆 \\
\hline BSM & Biological Sciences Museum, Hong Kong University, Hong Kong, CHINA & 香港大学生物科学博物馆 \\
\hline CAF & Insect Museum of Chinese Academy of Forestry, Beijing, CHINA & 中国林业科学研究院昆虫标本馆 \\
\hline CAS & California Academy of Science, San Francisco, California, USA & 美国旧金山加利福尼亚科学院 \\
\hline $\mathrm{CNC}$ & $\begin{array}{l}\text { the Canadian National Collection of Insects, Arachnids and Nematodes, Ottawa, } \\
\text { CANADIA }\end{array}$ & 加拿大国家昆虫、蛛形纲动物和线虫标本馆 \\
\hline CNUB & College of Life Sciences, Capital Normal University, Beijing, CHINA & 首都师范大学生命科学学院 \\
\hline CQNU & Chongqing Normal University, Chongqing, CHINA & 重庆师范大学 \\
\hline CSCS & Central South University of Forestry and Technology, Changsha, CHINA & 中南林业科技大学 \\
\hline DNUE & Daegu National University of Education, Science Education, Daegu, SOUTH KOREA & 韩国大邱国立教育大学 \\
\hline EBRRS & the Emeishan Biological Resources Research Station, Leshan, CHINA & 峨眉山生物资源实验站 \\
\hline FAFU & Fujian Agriculture and Forestry University, Fuzhou, CHINA & 福建农林大学 \\
\hline FBUB & Institute of Zoology, Faculty of Biology, University of Belgrade, Belgrade, SERBIA & 贝尔格莱德大学生物学院动物学研究所 \\
\hline GSFGPM & $\begin{array}{l}\text { General Station of Forest and Grassland Pest Management, National Forestry and } \\
\text { Grassland Administration, Shenyang, CHINA }\end{array}$ & 国家林业和草原局森林和草原病虫害防治总站 \\
\hline GXNU & Insect Collection of Guangxi Normal University, Guilin, CHINA & 广西师范大学昆虫标本馆 \\
\hline
\end{tabular}




\begin{tabular}{|c|c|c|}
\hline $\begin{array}{l}\text { 缩写 } \\
\text { Abbreviation }\end{array}$ & $\begin{array}{l}\text { 英文全称 } \\
\text { Full Form in English }\end{array}$ & $\begin{array}{l}\text { 中文全称 } \\
\text { Full Form in Chinese }\end{array}$ \\
\hline HNHM & $\begin{array}{l}\text { the Hymenoptera Collection of the Hungarian Natural History Museum, Budapest, } \\
\text { HUNGARY }\end{array}$ & 匈牙利国家历史博物馆膜翅目标本馆 \\
\hline IBBL & $\begin{array}{l}\text { Insect Biodiversity and Biogeography Lab (IBBL), School of Biological Sciences, Hong } \\
\text { Kong University, Hong Kong, CHINA }\end{array}$ & $\begin{array}{l}\text { 香港大学生物科学学院生物多样性与生物地理实 } \\
\text { 验室 }\end{array}$ \\
\hline IBSS & $\begin{array}{l}\text { Federal Scientific Center of the East Asia Terrestrial Biodiversity (formerly Institute of } \\
\text { Biology and Soil Science), Vladivostok, RUSSIA }\end{array}$ & 俄罗斯东亚陆地生物多样性联邦科学中心 \\
\hline ICXU & Xinjiang University, Urumqi, CHINA & 新疆大学 \\
\hline IEBR & Institute of Ecology and Biological Resources, Hanoi, VIETNAM & 越南河内生态与生物资源研究所 \\
\hline IZCAS & Institute of Zoology, Chinese Academy of Sciences, Beijing, CHINA & 中国科学院动物研究所 \\
\hline KIZ & Kunming Institute of Zoology, Chinese Academy of Science, Kunming, CHINA & 中国科学院昆明动物研究所 \\
\hline KUEC & $\begin{array}{l}\text { Entomological Laboratory and Institute of Tropical Agriculture, Kyushu University, } \\
\text { Fukuoka, JAPAN }\end{array}$ & 日本九州大学热带农业研究所昆虫学研究室 \\
\hline LSAF & $\begin{array}{l}\text { Scientific Research and Management Center of East China Pharmaceutical Botanical } \\
\text { Garden, Lishui, CHINA }\end{array}$ & 丽水华东药用植物园 \\
\hline MZLU & the Biological Museum of Lund University, Lund, SWEEDEN & 瑞典隆德大学生物标本馆 \\
\hline NAU & Nanjing Agricultural University, Nanjing, CHINA & 南京农业大学 \\
\hline NCHUC & Chun Hsing University Collection, Taiwan, CHINA & 中兴大学标本馆 (台湾) \\
\hline NEFU & Northeast Forestry University, HaErBin, CHINA & 东北林业大学 \\
\hline NIGPAS & $\begin{array}{l}\text { Nanjing Institute of Geology and Paleontology, Chinese Academy of Sciences, Nanjing, } \\
\text { CHINA }\end{array}$ & 中国科学院南京地质古生物研究所 \\
\hline
\end{tabular}




\begin{tabular}{|c|c|c|}
\hline 缩写 & 英文全称 & 中文全称 \\
\hline Abbreviation & Full Form in English & Full Form in Chinese \\
\hline NWUX & $\begin{array}{l}\text { the collections in the Department of Life Sciences of the Northwest University, Xi An, } \\
\text { CHINA }\end{array}$ & 西北大学生命科学学院标本馆 \\
\hline OLML & Oberoesterreichisches Landesmuseum Linz, AUSTRA & 奥地利林茨国立博物馆 \\
\hline RMNH & Naturalis Biodiversity Center (RMNH), Leiden, NETHERLANDS & 荷兰莱顿博物学生物多样性中心 \\
\hline SCAU & South China Agricultural University, Guangzhou, CHINA & 华南农业大学 \\
\hline SMNG & Senckenberg Museum für Naturkunde, Görlitz, GERMANY & 德国格尔利茨森肯贝格自然历史博物馆 \\
\hline SNU & $\begin{array}{l}\text { College for Agriculture and Life Sciences, Seoul National University, Seoul, SOUTH } \\
\text { KOREA }\end{array}$ & 韩国首尔国立大学农业与生命科学学院 \\
\hline SYSBM & The Museum of Biology, Sun Yat-sen University, Guangzhou, CHINA & 中山大学生物标本馆 \\
\hline TJAU & Tianjin Agricultural University, Tianjin, CHINA & 天津农学院 \\
\hline TNMNS & Museum of Natural Science, Taiwan, CHINA & 台湾自然科学博物馆 \\
\hline UB & University of Barcelona, Barcelona, SPAIN & 西班牙巴塞罗那大学 \\
\hline USNM & National Museum of Natural History, Smithsonian Institution, Washington DC, USA & 美国国家自然历史博物馆 \\
\hline YNAU & Yunnan Agricultural University, Kunming, CHINA & 云南农业大学 \\
\hline ZIN & $\begin{array}{l}\text { the collection of the Zoological Institute of the Russian Academy of Sciences, St. } \\
\text { Petersburg, RUSSIA }\end{array}$ & 俄罗斯科学院动物研究所标本馆 \\
\hline ZJU & Zhejiang University, Hangzhou, CHINA & 浙江大学 \\
\hline
\end{tabular}




\begin{tabular}{|c|c|c|c|}
\hline & $\begin{array}{l}\text { 期刊或出版社名称 } \\
\text { Journal or Publisher }\end{array}$ & $\begin{array}{l}\text { 发表文章数 } \\
\text { 量 (篇) } \\
\text { Number of } \\
\text { Publications }\end{array}$ & $\begin{array}{l}\text { 发表新物种 } \\
\text { 数量 (个) } \\
\text { Number of } \\
\text { New Species } \\
\text { Published } \\
\end{array}$ \\
\hline 1 & Acta Zoologica Academiae Scientiarum & 1 & 1 \\
\hline 2 & Alcheringa: An Australasian Journal of Palaeontology & 2 & 10 \\
\hline 3 & Alpine Entomology & 1 & 1 \\
\hline 4 & Asian Myrmecology & 1 & 4 \\
\hline 5 & Biodiversity Data Journal & 1 & 0 \\
\hline 6 & Bulletin of Entomological Research & 1 & 0 \\
\hline 7 & Bulletin of the Kyushu University Museum & 1 & 2 \\
\hline 8 & Caucasian Entomological Bulletin & 1 & 2 \\
\hline 9 & Deutsche Entomologische Zeitschrift & 1 & 11 \\
\hline 10 & $\begin{array}{l}\text { Entomotaxonomia } \\
\text { 昆虫分类学报 }\end{array}$ & 9 & 19 \\
\hline 11 & European Journal of Taxonomy & 1 & 5 \\
\hline 12 & Far Eastern Entomologist & 2 & 2 \\
\hline 13 & $\begin{array}{l}\text { Forest Pest and Disease } \\
\text { 中国森林病虫 }\end{array}$ & 1 & 0 \\
\hline 14 & Insect Systematics \& Evolution & 1 & 3 \\
\hline 15 & Insects & 2 & 3 \\
\hline 16 & Journal of Asia-Pacific Entomology & 3 & 3 \\
\hline 17 & $\begin{array}{l}\text { Journal of Environmental Entomology } \\
\text { 环境昆虫学报 }\end{array}$ & 1 & 0 \\
\hline 18 & $\begin{array}{l}\text { Journal of Forestry Research } \\
\text { 林业研究 }\end{array}$ & 1 & 1 \\
\hline 19 & Journal of Hymenoptera Research & 7 & 13 \\
\hline 20 & Journal of Natural History & 3 & 4 \\
\hline 21 & $\begin{array}{l}\text { Journal of Northeast Forestry University } \\
\text { 东北林业大学学报 }\end{array}$ & 2 & 2 \\
\hline 22 & Journal of Systematic Palaeontolog & 1 & 0 \\
\hline 23 & Opuscula Zoologica Budapest & 1 & 3 \\
\hline 24 & Oriental Insects & 1 & 1 \\
\hline 25 & Phytoparasitica & 1 & 2 \\
\hline 26 & $\begin{array}{l}\text { Proceedings of the Entomological Society of } \\
\text { Washington }\end{array}$ & 4 & 3 \\
\hline 27 & Science Press 科学出版社 & 1 & 81 \\
\hline 28 & $\begin{array}{l}\text { Scientia Silvae Sinicae } \\
\text { 林业科学 }\end{array}$ & 2 & 2 \\
\hline 29 & Sociobiology & 1 & 1 \\
\hline 30 & Soil Organisms & 1 & 4 \\
\hline 31 & $\begin{array}{l}\text { Southwest China Journal of Agricultural Sciences } \\
\text { 西南农业学报 }\end{array}$ & 1 & 0 \\
\hline 32 & ZooKeys & 16 & 17 \\
\hline 33 & Zoologia & 1 & 1 \\
\hline 34 & $\begin{array}{l}\text { Zoological Systematics } \\
\text { 动物分类学报 }\end{array}$ & 4 & 5 \\
\hline 35 & Zoosystematica Rossica & 1 & 1 \\
\hline \multirow[t]{2}{*}{36} & Zootaxa & 26 & 114 \\
\hline & Total 共计 & 105 & 321 \\
\hline
\end{tabular}

